\section{AEE RESEARCH and}

DEVELOPMENT REPORT

\section{PERT-IV, A TWO-DIMENSIONAL PERTURBATION CODE IN FORTRAN-IV}

R. W. HARDIE

W. W. LITTLE, JR.

APRIL 1967

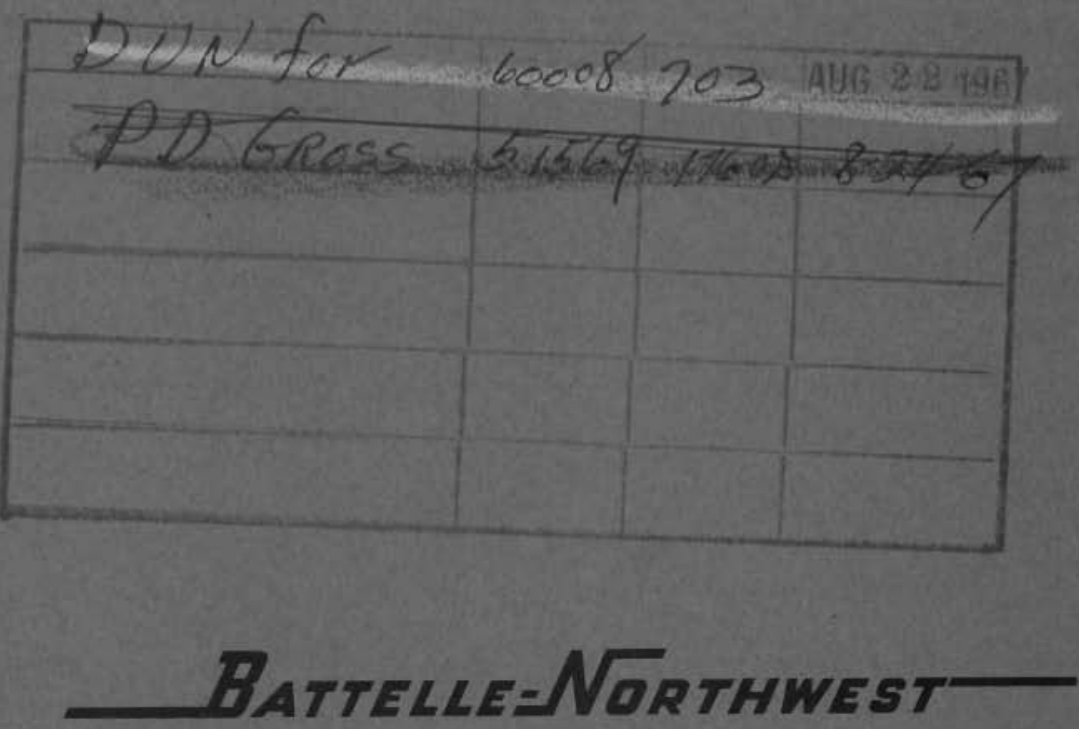

BATTELLE MEMORIAL INSTITUTE / PACIFIC NORTHWEST LABORATORY 


\section{LEGAL NOTICE}

This report was prepared as an account of Government sponsored work. Neither the United States, nor the Commission, nor any person acting on behalf of the Commission:

A. Makes any warranty or representation, expressed or implied, with respect to the accuracy, completeness, or usefulness of the information contained in this report, or that the use of any information, apparatus, method, or process disclosed in this report may not infringe privately owned rights; or

B. Assumes any liabilities with respect to the use of, or for damages resulting from the use of any information, apporatus, method, or process disclosed in this report.

As used in the above, "person acting on behalf of the Commission" includes any employee or contractor of the Commission, or employee of such contractor, to the extent that such employee or con. tractor of the Commission, or employee of such contractor prepares, disseminates, or provides access to, any information pursuant to his employment or contract with the Commission, or his employment with such contractor.

PACIFIC NORTHWEST LABORATORY

RICHLAND, WASHINGTON

operated by

BATTELLE MEMORIAL INSTITUTE

for the

UNITED STATES ATOMIC ENERGY COMMISSION UNDER CONTRACT AT(45-1)-1830 


\title{
33679000605552
}

i

$$
\begin{gathered}
\text { BNWL-409 } \\
\text { UC-32, Mathematics } \\
\text { and Computers }
\end{gathered}
$$

\author{
PERT-IV, A TWO-DIMENSIONAL \\ PERTURBATION CODE IN FORTRAN-IV \\ By \\ R. W. Hardie and W. W. Little, Jr. \\ FFTF Reactor Physics Section \\ Reactor Physics Department
}

\section{April 1967}

FIRET OMRESTRIGTED MAY
DISTRIBUTION MADE

PACIFIC NORTHWEST LABORATORY

RICHLAND, WASHINGTON 
Printed in the United States of America Available from

Clearinghouse for Federal Scientific and Technical Information National Bureau of Standards, U.S. Department of Commerce

Springfield, Virginia 22151

Price: Printed Copy $\$ 3.00$; Microfiche $\$ 0.65$ 


\section{PERT-IV, A TWO-DIMENSIONAL PERTURBATION CODE IN FORTRAN-IV \\ $\underline{\text { ABSTRACT }}$}

PERT-IV is a two-dimensional (R-Z, X-Y, R- $\theta$ ), firstorder perturbation theory code for computing reactivity coefficient traverses, activity traverses, the neutron generation time, and the effective delayed neutron fraction. The input flux and adjoint flux can be taken directly from 2-D calculations or synthesized (by the code) from 1-D calculations.

In a $65 \mathrm{~K}$ memory (and 4 tapes), the code can handle 35 materials, 26 energy groups, and a 50 x 50 spatial mesh. Running time on a UNIVAC 1107 is about 3 minutes.

Input instructions, a sample case, and a FORTRAN-IV source deck 1 isting are given in the appendices. 
TABLE OF CONTENTS

I. INTRODUCTION . . . . . . . . . . . . . . . . . . 1

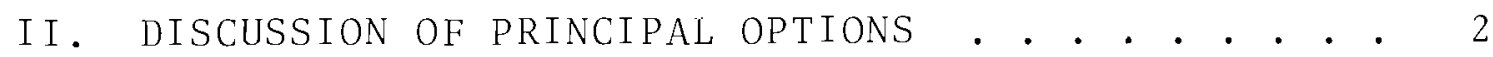

Format of Input Fluxes and Adjoint Fluxes . . . . 2

Flux Normalization Options . . . . . . . . . 2

Activity Traverses . . . . . . . . . . . . . 2

Reactivity Coefficient Calculation . . . . . . 3

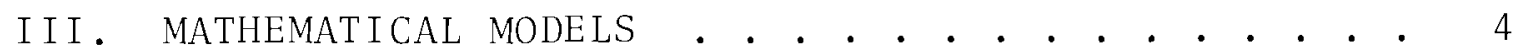

IV. LimitS ON PROBLEM COMPLEXITY . . . . . . . . . . . 6

REFERENCES ................. . 6

APPENDIX A: inPUT instRUCtIONS . . . . . . . . . A-1

APPENDIX B: SAMPLE PROBlem . . . . . . . . . . . B-1

APPENDIX C: FORTRAN-IV SOURCE DECK LISTING $\ldots$. . C-1 


\section{PERT-IV, A TWO-DIMENSIONAL PERTURBATION CODE IN FORTRAN-IV \\ R. W. Hardie and W. W. Little, Jr. \\ I. INTRODUCTION}

This report describes a two-dimensional perturbation code for use in fast reactor analysis. The code can be used to:

- Compute reactivity coefficient traverses using first order perturbation theory

- Compute activity traverses for any cross section of any material

- Compute the neutron generation time and the effective delayed neutron fraction.

The code is compatible with the one-dimensional DTF-IV (1) and two-dimensional $2 \mathrm{DF}{ }^{(2)}$ transport theory codes from Los Alamos. In fact, the generalized input portions of these prograns were incorporated into the PERT-IV code. 


\section{DISCUSSION OF PRINCIPAL OPTIONS}

\section{Format of Input Fluxes and Adjoint Fluxes}

The input flux and adjoint flux options are:

- Flux and adjoint from DTF-IV

- Flux and adjoint from 2DF

- Flux from DTF-IV, adjoint from 2DF

- Flux from 2DF, adjoint from DTF-IV

- Flux from DTF-IV, no adjoint

- Flux from 2DF, no adjoint

When 1-D flux dumps are used, PERT-IV synthesizes these into 2-D arrays. For the last two options, the code assumes a flat adjoint distribution.

Flux Normalization Options

Four options are available for normalizing the flux. These are:

- Center point flux

- Center point power density

- Total reactor power

- Denominator of perturbation equation [see Equation (1)].

The adjoint flux is always normalized such that the fission source times the adjoint, summed over all groups, is unity at the center mesh point. 


\section{Reactivity Coefficient Calculation}

Using first-order perturbation theory, PERT-IV will calculate reactivity coefficient (in units of $\delta \mathrm{k} / \mathrm{kg}$ ) traverses for any specified row or column. The contribution of each component of the reactivity coefficient (e.g., moderation, capture) is also given.

\section{Activity Traverses}

For any material and cross section (e.g., $\left.\sigma_{f}, \sigma_{a}\right)$, the code will calculate and print a complete activity map. A full print gives the activity at each mesh point for each group; a partial print gives the total over all groups at each mesh point. Zone averaged activities are also given. 


\section{MATHEMATICAL MODELS}

Let: $\quad \mathrm{N}=$ number of energy groups,

$$
\begin{aligned}
& \phi_{i}=\text { flux in group i }\left(\mathrm{n}-\mathrm{cm}^{-2}-\mathrm{sec}^{-1}\right) \text {, } \\
& \phi_{i}^{\dagger}=\operatorname{adjoint} \text { flux in group } i, \\
& v_{i}=\text { neutron velocity in group } i\left(\mathrm{~cm}-\mathrm{sec}^{-1}\right) \text {, } \\
& x_{i}=\text { fission source in group } i \text {, } \\
& v \Sigma_{f}^{i}=\text { macroscopic fission source cross section in } \\
& \text { group i }\left(\mathrm{cm}^{-1}\right) \text {, } \\
& \Sigma_{a}^{i}=\text { macroscopic absorption cross section in } \\
& \text { group i }\left(\mathrm{cm}^{-1}\right) \text {, } \\
& \Sigma_{t r}^{i}=\text { macroscopic transport cross section in } \\
& \text { group i }\left(\mathrm{cm}^{-1}\right) \text {, } \\
& \Sigma(i \rightarrow j)=\text { macroscopic transfer cross section from } \\
& \text { group } i \text { to } j\left(\mathrm{~cm}^{-1}\right) \text {, } \\
& \mathrm{k} \text { = effective multiplication factor, }
\end{aligned}
$$

where, for simplicity, the spatial variables are not explicitly shown.

The reactivity coefficients at each mesh point are computed using the equation

$$
\frac{\delta \mathrm{k}}{\mathrm{k}}=\frac{\mathrm{A}+\mathrm{B}+\mathrm{C}+\mathrm{D}}{\mathrm{I}},
$$

where:

$$
\begin{aligned}
& I=\frac{1}{k} \int_{\text {Reactor }}^{d V}\left\{\sum_{j=1}^{N}\left(x_{j} \phi_{j}^{+}\right) \sum_{i=1}^{N}\left(u \Sigma_{f}^{i}{ }_{i}\right)\right\}, \\
& A=\int d V\left\{\sum_{i=1}^{N}\left(\overrightarrow{\nabla \phi}_{i} \cdot \overrightarrow{\nabla \phi}_{i}^{\dagger}\right) \frac{\delta\left[\Sigma_{t r}^{i}\right]}{3\left(\Sigma_{t r}^{i}\right)^{2}}\right\},
\end{aligned}
$$




$$
\begin{aligned}
& B=\frac{1}{k} \int d V\left\{\sum_{j=1}^{N}\left(x_{j} \phi_{j}^{+}\right) \sum_{i=1}^{N}\left(\delta\left[v \Sigma_{f}^{i}\right] \phi_{i}\right)\right\}, \\
& C=-\int d V\left\{\sum_{i=1}^{N}\left(\delta\left[\Sigma_{a}^{i}\right] \phi_{i} \phi_{i}^{\dagger}\right)\right\}
\end{aligned}
$$

and

$$
D=\int d v\left\{\sum_{i=1}^{N}\left(\sum_{j=i+1}^{N} \delta[\Sigma(i \rightarrow j)] \phi_{i}\left(\phi_{j}^{+}-\phi_{i}^{+}\right)\right)\right\} .
$$

The gradient terms in Equation (3) are evaluated by using first order difference approximations; the gradients at $r=0$ (left boundary) are assumed zero.

The neutron generation time is calculated from the expression

$$
\Lambda=\frac{1}{k} \int \mathrm{dV}\left\{\sum_{i=1}^{N} \phi_{i} \phi_{i}^{+} / v_{i}\right\} / \mathrm{I}
$$

The effective delayed neutron fraction is computed using the algorithm

$$
\beta_{\text {eff }}=\frac{1}{k} \int d V\left\{\sum_{i=1}^{N}\left(\sum_{\ell=1}^{L} f_{\ell} \Sigma_{f, \ell}^{i}\right) \phi_{i} \sum_{j=1}^{N} x_{j}^{D_{j}^{+}}\right\} / I \text {, }
$$

where:

$$
L=\text { number of fissionable isotopes for } \beta_{\text {eff }}
$$
calculation,

$f_{\ell}=$ absolute delayed neutron yield per fission of isotope $\ell$,

$\begin{aligned} \Sigma_{f, \ell}^{i}= & \text { macroscopic fission cross section in group } i \\ & \text { of isotope } \ell\left(\mathrm{cm}^{-1}\right),\end{aligned}$

$x_{j}^{D}=$ delayed fission source in group $j$. 
IV. LIMITS ON PROBLEM COMPLEXITY

\begin{tabular}{|c|c|c|}
\hline Variable & Definition & Value \\
\hline IGM & No. of Energy Groups & 26 \\
\hline IST & No. of Downscattering Terms & 10 \\
\hline IM & No. of Radial Intervals & 50 \\
\hline JM & No. of Axial Intervals & 50 \\
\hline MCR & No. of Cross Sections from Cards & 25 \\
\hline MTP & No. of Cross Sections from Tape & 25 \\
\hline $\mathrm{IZM}$ & No. of Material Zones & 35 \\
\hline MT & No. of Materials Including Mixes & 35 \\
\hline M01 & No. of Mixture Specifications & 45 \\
\hline NDELK & No. of Reactivity Traverses & 20 \\
\hline NIBC & No. of Isotopes in $\beta_{\text {eff }}$ Calculation & 20 \\
\hline NACT & No. of Activity Traverses & 3 \\
\hline
\end{tabular}

\section{REFERENCES}

1. K. D. Lathrop. DTF-IV, a FORTRAN-IV Program for Solving $\frac{\text { the Multigroup Transport Equation with Anisotropic }}{\text { Scattering, LA-3373, Los Alamos, New Mexico. } 1965 .}$

2. 2DF, A Two-Dimensional Sn Code. Unpublished Data. Los Alamos Scientific Laboratory. 


\section{APPENDIX A}

\section{INPUT INSTRUCTIONS}

The following two pages describe the input data for PERT - IV .

For DTF fluxes and all subsequent data, the cards consist of six data fields of 12 columns each. The last nine columns contain the data associated with the particular field; columns 2-3 contain an integer, $N$, from 0 (or blank) to 99. The first column of each field must contain:

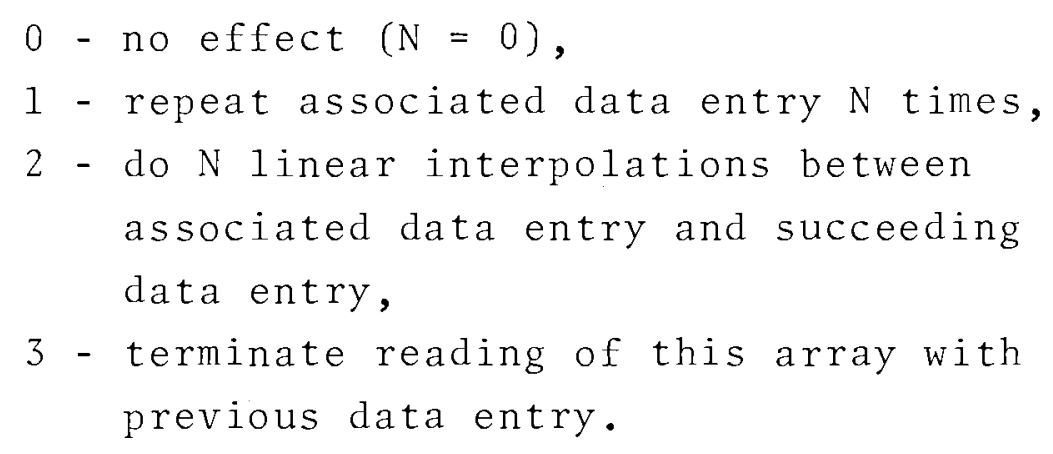




\section{PERT - IV}

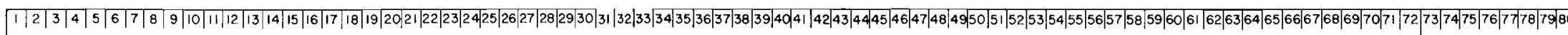

identification card

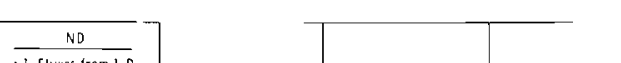

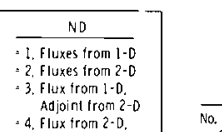

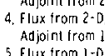

$\frac{16 M}{\text { No. of Energy }}$

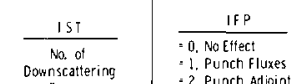

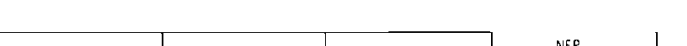

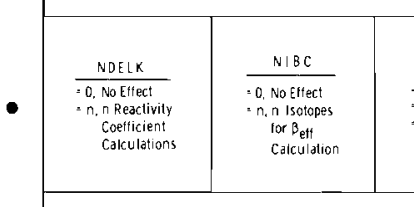

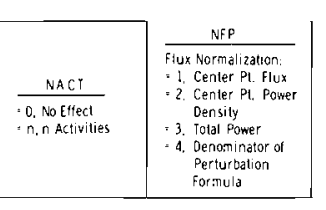
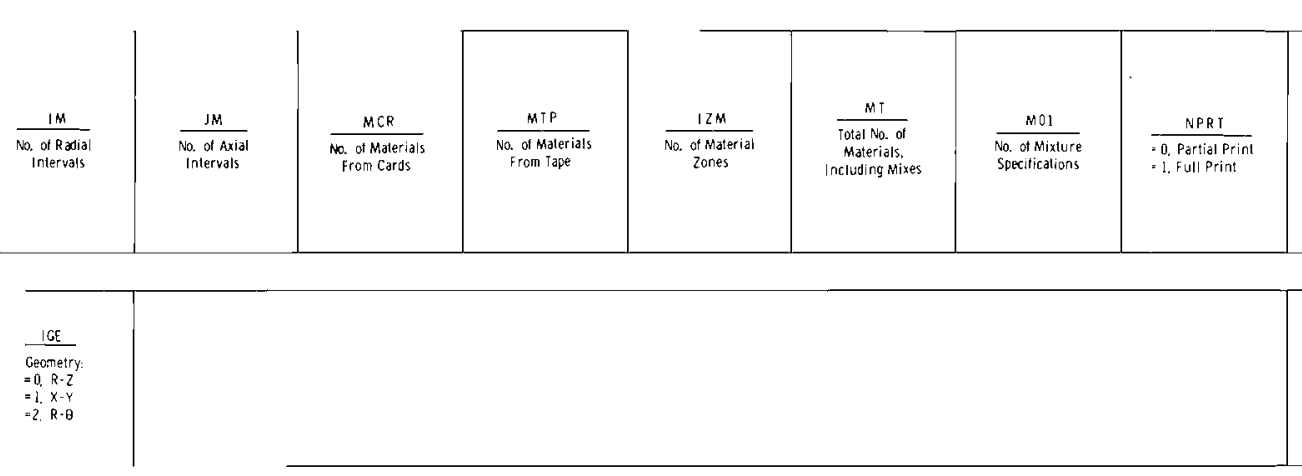

- $\frac{F L P O}{\text { flux or Poner (See NFP) }}$

$\frac{\mathrm{VF}}{\text { volume factor }}$
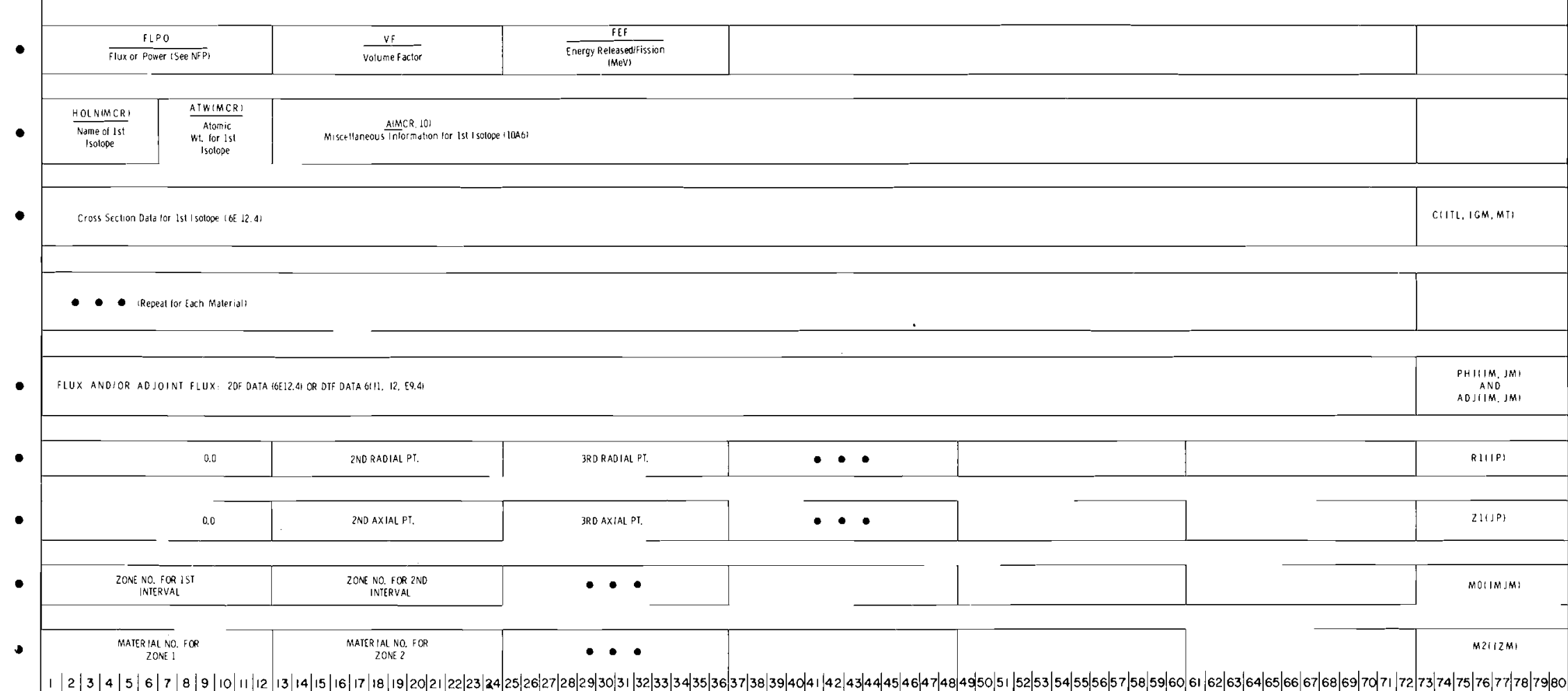

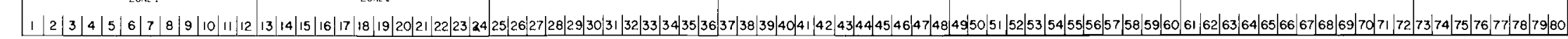




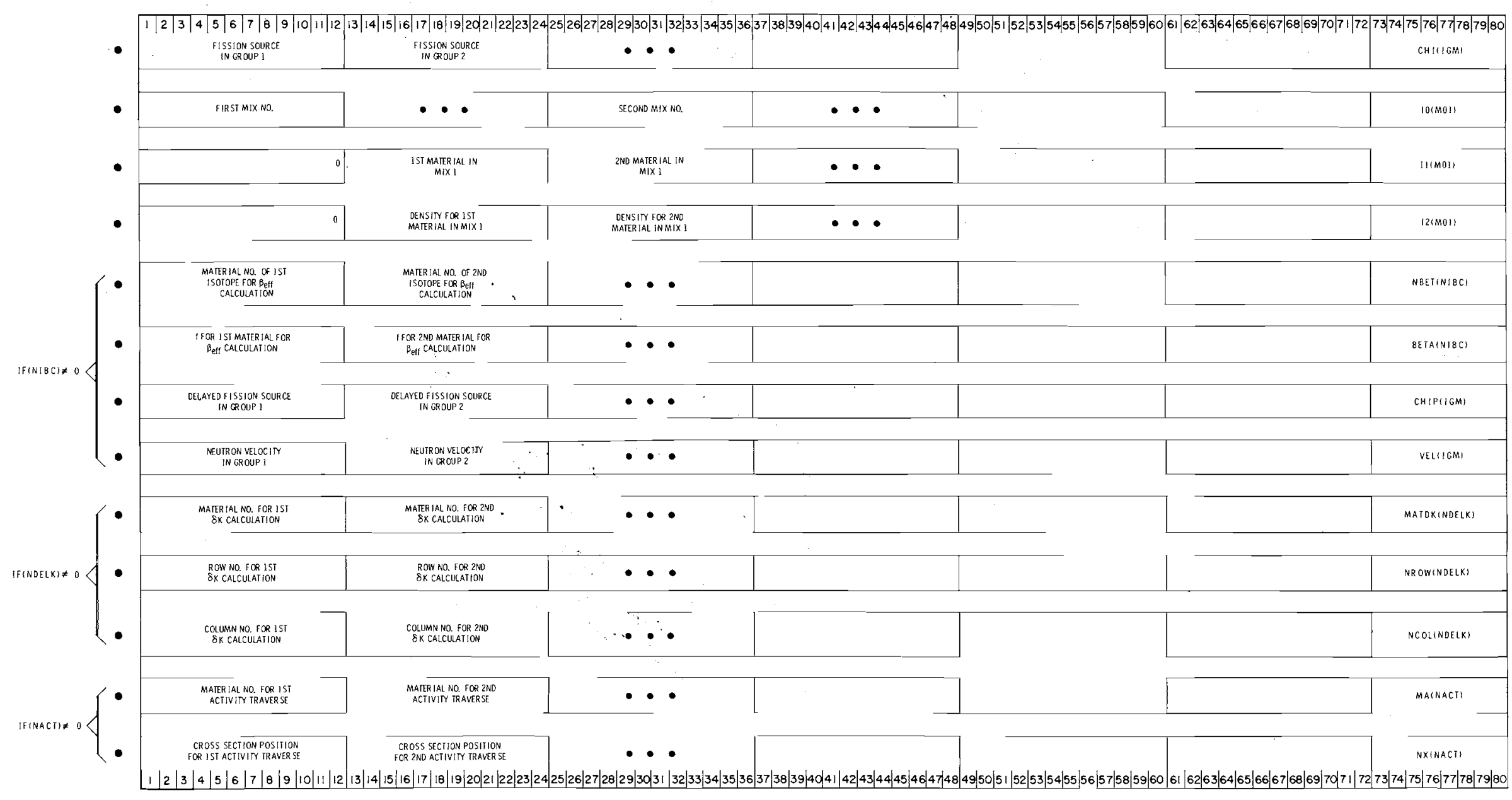




\section{APPENDIX B \\ SAMPLE PROBLEM}

The following pages show the input data (omitting input fluxes) and computer output for a 2-group, 3-region test problem in R-Z geometry. 


\begin{tabular}{|c|c|c|c|c|c|c|}
\hline$E R T-I V$ & EST & $S E$ & (20) & LUX INP & $2 \mathrm{GF}$ & \\
\hline 2 & 2 & 0 & 21 & 16 & 19 & 31 \\
\hline 3 & 6 & 1 & 0 & & & \\
\hline \multicolumn{7}{|c|}{$2 P \cup 49239.05$} \\
\hline & +01 & . $184072+U 1$ & - Dl1bu3+ul & $.669364+U 1$ & $.476589+01$ & .000000 \\
\hline .228414 & $y+u 1$ & . 324 vuotu1 & $.649503+01$ & $.135176+02$ & $.10677 b+02$ & $.790403-$ \\
\hline \multicolumn{7}{|c|}{00240240.05} \\
\hline $\begin{array}{l}.697466 \\
.205185\end{array}$ & & $\begin{array}{l}.973 u 4 b-v u \\
.158456+U 1\end{array}$ & $\begin{array}{l}.21 \cup 364+01 \\
.577565-01\end{array}$ & $\begin{array}{l}.634004+01 \\
.139455+02\end{array}$ & $\begin{array}{l}.549212+01 \\
.123609+02\end{array}$ & $\begin{array}{l}00 \\
43-01\end{array}$ \\
\hline \multicolumn{7}{|c|}{$M 2 \cup 235235 \cdot(14$} \\
\hline $\begin{array}{l}.132033 \\
.336017\end{array}$ & $\begin{array}{l}+01 \\
7+01\end{array}$ & $\begin{array}{l}.150>65+01 \\
.48697<+01\end{array}$ & $\begin{array}{l}.3324 \\
.8149\end{array}$ & $.022874+01$ & $.457201+01$ & .000 \\
\hline 38238 & 8.05 & 2 GROUP & & & & \\
\hline - IUUIOU & $v+v u$ & $.232887-v u$ & $.281437-00$ & $.633569+01$ & $.600524+01$ & .00 \\
\hline - vuvuou & & . $532188-u v$ & . vuUuvu & . $131=03+02$ & $51+02$ & . \\
\hline \multicolumn{7}{|c|}{2 GROUPS } \\
\hline - vucuúa & & $.833620-U S$ & .000000 & 26342 & $3+01$ & .00 \\
\hline \multirow{2}{*}{\multicolumn{2}{|c|}{$\begin{array}{l}\text { - unuvuu } \\
22.990\end{array}$}} & $.456935-1 v$ & .000 & & $+e_{0}$ & \\
\hline & & \multicolumn{5}{|c|}{2 GROUPS } \\
\hline . unuvou & & $.713 \cup \cup 6-\cup 3$ & .000 & 30 & .30 & .00 \\
\hline - uvuuuu & & $.423424-v 2$ & .000 & 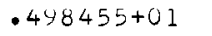 & .491 & \\
\hline \multicolumn{7}{|c|}{ M2 FE 55.847} \\
\hline $\begin{array}{l}\text { - vudovou } \\
\text { - vuvuou }\end{array}$ & & - $591775-02$ & .000000 & 25576 & $+5+01$ & .00 \\
\hline \multicolumn{7}{|l|}{$C R \quad 5$} \\
\hline - vuuuvu & & $.45449 u-v 2$ & .000 & 01 & 20 & 0 \\
\hline \multicolumn{7}{|l|}{ NI $\quad 58.7$} \\
\hline - uvuvou & & . $3 \cup 5<71-\cup 1$ & - uuvcuu & .30 & $5+01$ & \\
\hline \multicolumn{7}{|c|}{ SS } \\
\hline - cucuuác & & $.43 \cup 943-\cup 1$ & .000 & & .51 & \\
\hline - UUUบOi & & . $3 \cup 3487-v u$ & .000 & & & \\
\hline \multicolumn{7}{|l|}{ AL } \\
\hline $\begin{array}{l}.000 v 00 \\
.000000\end{array}$ & & - $205447-02$ & .000000 & .28 & .27 & \\
\hline \multicolumn{7}{|c|}{2 GROUPS } \\
\hline $\begin{array}{r}.134241 \\
.211774\end{array}$ & $\begin{array}{l}1+01 \\
y+01\end{array}$ & $\begin{array}{l}.15691 b+01 \\
.283435+01\end{array}$ & $\begin{array}{l}.333434+01 \\
.515408+01\end{array}$ & & $4+$ & .0 \\
\hline \multicolumn{7}{|c|}{ U238 238.05} \\
\hline .372181 & & - $10 y b 16-v u$ & $78+0 u$ & .69 & .66 & \\
\hline $.000 \cup 00$ & & $.404503-00$ & .000000 & 2 & 1 & $1+1$ \\
\hline \multirow{2}{*}{\multicolumn{2}{|c|}{$\begin{array}{l}\text { M2 FE } 55.847 \\
\text {. OUUUUU }\end{array}$}} & 2 GROUP & & & & \\
\hline \multirow{3}{*}{\multicolumn{2}{|c|}{$C R^{.0 U 0000} \quad 51.996$}} & $\begin{array}{l}u 574-v 2 \\
u 269-v 1\end{array}$ & $\begin{array}{l}.000000 \\
.000000\end{array}$ & $i$ & & 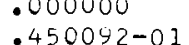 \\
\hline & & $\begin{array}{l}2 \text { GROU } \\
21-U 2\end{array}$ & 5 & 01 & $7499+01$ & 10 \\
\hline & & & & & & \\
\hline
\end{tabular}




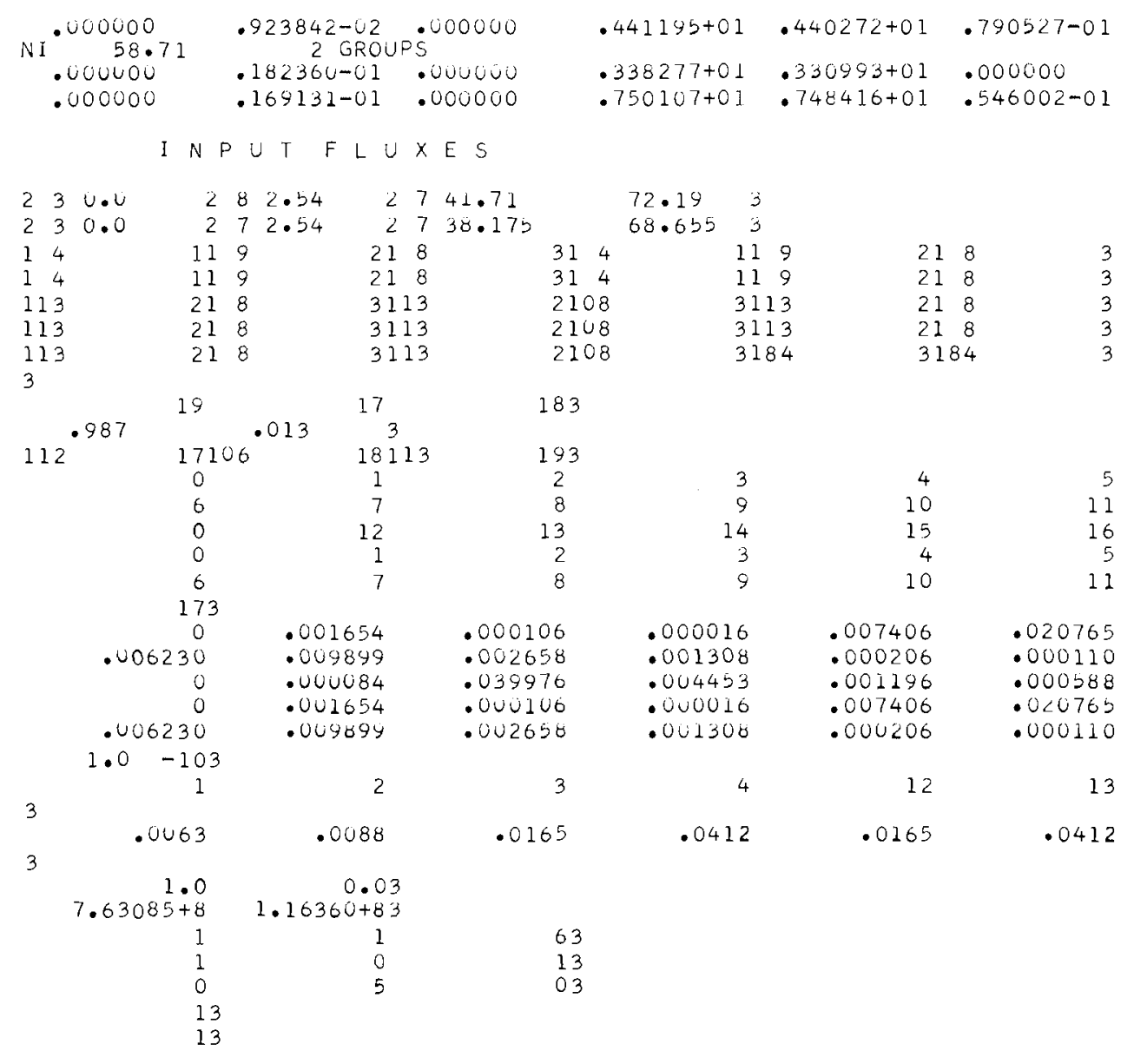


NDELK NIBC

NACT

NACT

IGE

FLPO

$V F$
$F E F$

1/2/3/4/5/GEFLUXES FROM TWO 1-D RUNS/FLUXES FROM 2-D RUN/FLUX FROM 1-D.ADJOINT FROM 2-D/FLUX FROM 2-D, ADJOINT FROM 1-D/FLUX FROM 1-D. NO ADJOINT/FLUX FROM 2-D.NO ADJOINT

NUMBER OF ENERGY GROUPS

NUMBER OF DOWNSCATTERING TERMS

$0 / 1 / 2=$ NO EFFECT/PUNCH FLUXES/PUNCH ADJOINT FLUXES

NUMBER OF RADIAL INTERVALS

NUMBER OF AXIAL INTERVALS

NUMBER OF WATERIALS FROM CAROS

NUMBER OF MATERIALS FROM TAPE

NUMBER OF MATERIAL TONES

TOTAL NUNIAER OF MATERIALS INCLUDING WIXES

IVUMBER OF MIXTURE SPECIFICATIONS

PRINT OPTION $(0 / 1=P A R T I A L$ PRINT/FULL PRINT)

O/N=NO EFFECT/DO N REACTIVITY COEFFICIENT CALCULATIONS

$O / N=N O$ EFFECT/USE N MATERIALS FOR BETA EFFECTIVE CALCILATION

$O / N=N O$ EFFECT/DO N ACTIVITY TRAVERSES

FLUX OR PONER INORMALIZATION $(1 / 2 / 3 / 4=C E N T E R$ PT. FLUIX/CENTER PT.

POWER/TOTAL POWER /DENOMINATOR OF PERT FORMULA)

GEOMETRY (O/1/2=R-Z/X-Y/R-THETA)

\section{FLUX (N/CM2-SEC) OR POWER (NWT}

VOLUME FACTOR (VOLUME = VF*PI*R2*Z)

ENERGY (MEV) RELEASED PER FISSION

$1.00000+00$ $2.00000+00$ $2.06000+02$ 
THE FOLLOWING NUCLIDES ARE FROM CAROS

$\begin{array}{lll}1 & \text { M2PU49 } & 2 \text { GROUPS } \\ 2 & \text { PU240 } & 2 \text { GROUPS } \\ 3 & \text { M2U235 } & 2 \text { GROUPS } \\ 4 & \text { U238 } & 2 \text { GRQUPS } \\ 5 & \text { C } & 2 \text { GROUPS } \\ 6 & \text { NA } & 2 \text { GROUPS } \\ 7 & \text { M2 FE } & 2 \text { GROUPS } \\ 8 & \text { CR } & 2 \text { GROUPS } \\ 9 & \text { NI } & 2 \text { GROUPS } \\ 10 & \text { MO } & 2 \text { GROUPS } \\ 11 & \text { AL } & 2 \text { GROUPS } \\ 12 & \text { M2U235 } & 2 \text { GROUPS } \\ 13 & \text { U238 } & 2 \text { GROUPS } \\ 14 & \text { M2 FE } & 2 \text { GROUPS } \\ 15 & \text { CR } & 2 \text { GROUPS } \\ 16 & \text { NI } & 2 \text { GROUPS }\end{array}$




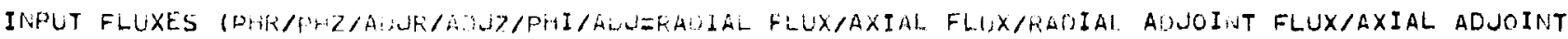

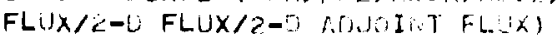

\begin{tabular}{|c|c|c|c|c|}
\hline $\begin{array}{r}\mathrm{PHI} \\
+822 c-03\end{array}$ & $\begin{array}{l}840 \\
.482\end{array}$ & .48 & -03 & $7 i-03$ \\
\hline $313787-03$ & $.20261-.3$ & $.29373-0.3$ & $.25665-03$ & $.10 .533=03$ \\
\hline $66700-05$ & $.48207-3$ & $.49261-03$ & $.45171-03$ & $.45118-03$ \\
\hline $34959-03$ & $.30777-13$ & $.26253-$ & - $21366-03$ & $.15660-03$ \\
\hline $12008-04$ & $.58744-15$ & $.43191-03$ & $.4 \cap 17<-63$ & $.+48136-0.3$ \\
\hline$=-03$ & $. .34937-1,3$ & .30 & .26 & $.21351-03$ \\
\hline $15-04$ & $.126,34-14$ & $.58692-05$ & $1-13$ & $.4 A \div \geqslant 3-03$ \\
\hline $41836-03$ & $.38632-3$ & $.34901-03$ & $.30720-33$ & $.26208-03$ \\
\hline $00-04$ & $.20895-: 4$ & .12 & .58 & $7-03$ \\
\hline $14-03$ & $.41444-3$ & .3 & $72-03$ & $.30436-03$ \\
\hline $37-04$ & $.320: 10-0.4$ & & $1-24$ & $.5 t$ \\
\hline $3 s-03$ & $.42877-13$ & .45 & -23 & $.33676-0.3$ \\
\hline $30-04$ & $.466901,-1.4$ & & & .12 \\
\hline $.43948 y-03$ & $.42821-3$ & .49 & .03 & $77-03$ \\
\hline $00-04$ & -4 & $.4:$ & & \\
\hline $63-03$ & $.41263-3$ & .4 & -03 & .3 \\
\hline $36-03$ & $.909983-14$ & $4-04$ & .4 & .279 \\
\hline $18-03$ & $.38277-.13$ & .3 & 3 & .353 \\
\hline $3-03$ & -13 & & & \\
\hline $37-03$ & -3 & .3 & .3 & .3 \\
\hline$b-03$ & .151 & - 1 & .7 & .50 \\
\hline $97-03$ & .2 & & & \\
\hline $21-03$ & $.16043-3$ & $\cdot 1$ & 04 & .0440 \\
\hline & .246 & & & .24 \\
\hline - 1 & $.156: 7-3$ & & 03 & $.7748 G=0$ \\
\hline $54-0$ & $.31495-5$ & .1 & 3 & \\
\hline $34-03$ & $.13248-3$ & .1 & .9 & $.78724-1$ \\
\hline & & & & \\
\hline $1-0$ & 3 & & & .67 \\
\hline $0-$ & -15 & & & \\
\hline & .7 & & & \\
\hline - $12667-04$ & $.89861-5$ & .6 & $520-35$ & .1778 \\
\hline $92-04$ & $A=114$ & .52 & $.48621-04$ & .43 \\
\hline 83-04 & $.3529-5$ & . $6 A 6544-05$ & & .292300 \\
\hline $.40867-04$ & $.39816-64$ & $.37146-04$ & $.35088-34$ & .33111 \\
\hline & .9360 & & & .351 \\
\hline .27 & & $.26262-$ & & .2367 \\
\hline .10177 & .818 & $.64100-05$ & $.48988-05$ & $.35656-6$ \\
\hline & 14 & 16 & .16125 & .15449 \\
\hline & 63370 & & & $106-6$ \\
\hline
\end{tabular}

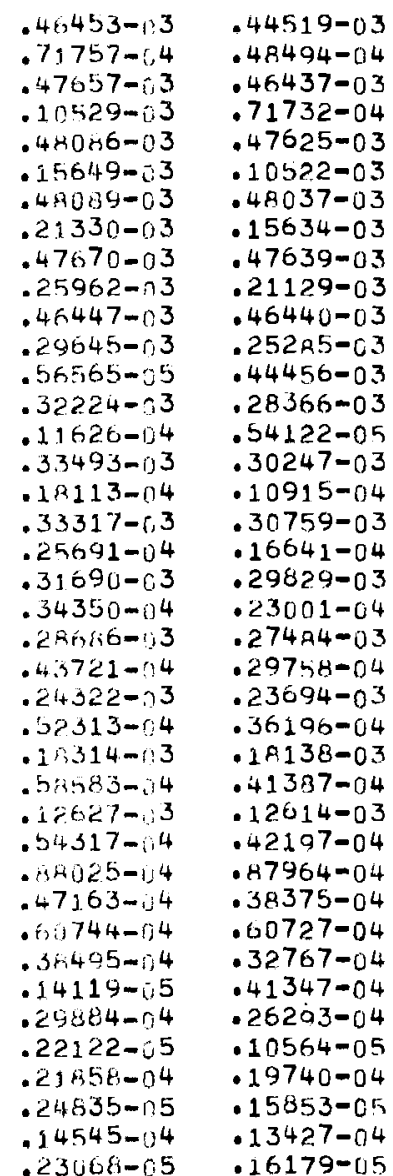

$.062453-133$ 35495-04 $.29884-04$ $.23068-05$
$.41920-03$ $.32370-04$ 44505-03 48477-04 $.46405-03$ $.71680-04$ $.47577-03$ $.10511-03$ $.47588-03$ $.15486-03$ .46408-03 $.20578-03$ $.44450-03$ $.24193-03$ $.41742-03$ . 26617-03 $.50827-05$ .27777-03 $.10033-04$ . $27529-03$ $.14918-04$ . 25866-03 . $9095-04$ $.19995-04$ $22701-03$ $.24860-04$ - $17670-03$ . $129071-04$ - $31706-04$ - $31706-04$ - $87864-04$ - $30262-04$ $.60681-04$ $.26004-04$ $.41334-04$ $.22459-04$ .27270-04 $.17391-04$ $.75639-06$ $.12134-04$ $.10283-05$
-38712-03 $.20945-04 \quad .12615-04$ $.41905-03$.38699-63 $.20934-04$ $.44477-03$.41876-03 $.48449-04 \quad .32341-04$ $.46358-03 \quad .44431-0$. $.71611-04 \quad .48404=04$ $.47133-03 \quad .45925-03$ $.10412-03 \quad .70937-04$ $.45916-03$ $.15082-03 \quad .10140=03$ $.44420-03 \quad .44371-0.3$ $.19687-03 \quad .14428=03$ $.41735=03 \quad .41708-03$ .22698-03 .18470-03 .38350-03 .38344-03 $.24437-0.3$.20829-03 $.46798-05 \quad .34342-03$ $.24852-0.3$.21854-03 $.90183-05 \quad .42050=05$ $.23867=03 \quad .21533=03$ $.78938-15$ $.21362-03 \quad 0319709-03$ .16836-04 $.16928-03$.15928-03 . $16928-03$.15940 . $12342-04$-13882-04 .12171-03 011660-03 $.22080-04$ - $16345-04$ .87027-04 .84790-04 . $23341-04 \quad .17407=04$ $.60613-04 \quad .60042-04$ $.21610-04 \quad .17021-04$ $.41305-04 \quad .41266=04$ $.18584-04 \quad .15047-04$ $.27263-04 \quad .27247-04$ -14924-04 $\quad 12432-04$ $.16746-04 \quad .16741=04$ $.10719-04 \quad .92220=05$ $.79327-05$ 


\begin{tabular}{|c|c|c|c|c|}
\hline & & & & \\
\hline .7929 & $.79277-0.5$ & $.79202-05$ & $.78430-05$ & $.76412-05$ \\
\hline $.43860-05$ & $.36835-5$ & $.30449-05$ & $.24703-05$ & $.19678-05$ \\
\hline $.23534=03$ & $.23527-13$ & $.23509-03$ & $.234 ? 4-03$ & $.23262-03$ \\
\hline $.14863-03$ & $.1256,4=03$ & -10065-03 & $.72208-04$ & $.47385-04$ \\
\hline $.21372-05$ & $.23526-13$ & $.23519-03$ & .23503003 & $.23477-03$ \\
\hline $.16964-03$ & $.14857-13$ & $.12559-03$ & $.10063-03$ & $.72184-04$ \\
\hline $.50930-05$ & $.21378-15$ & $.23512-03$ & $.23504-03$ & $.23486-03$ \\
\hline $.18817-03$ & $.16952-3$ & $.14847-03$ & $.22551-03$ & - joosa-03 \\
\hline $.87144-05$ & $.5091 n-5$ & $.21353=05$ & $.23487-03$ & $.23480-03$ \\
\hline $.20388-03$ & $.18797-3$ & $.16935-03$ & $.14833-03$ & $.12538-03$ \\
\hline $.13713=04$ & $.87070-5$ & $.50853-05$ & $.21332-05$ & $.23<65-03$ \\
\hline $.21465-03$ & $.20195-3$ & $.18620-03$ & $.26774-03$ & $.14691-03$ \\
\hline $.20655-04$ & $.13584-04$ & $.0624{ }^{2}-05$ & $.50371-05$ & $.21130-05$ \\
\hline $.21820-03$ & $.20905-13$ & $.19665=03$ & $.18132-03$ & $.16335-03$ \\
\hline $.30343=04$ & $.20114-4$ & $.13228-04$ & .0398305 & $.49054-05$ \\
\hline $.21422-03$ & $.20872-13$ & $.19996-03$ & $.18811-03$ & $.17342-03$ \\
\hline $.43606-04$ & $.29017-04$ & $.19234-04$ & . $12651-04$ & $.80335-05$ \\
\hline $.20283-03$ & $.20090-03$ & $.19574-03$ & $.288751-03$ & $.17639-03$ \\
\hline $.62278-04$ & $.40871-.14$ & $.27104-04$ & - $18031=04$ & $.11664-04$ \\
\hline $.18613-03$ & $.8593-3$ & $.15416-03$ & $.17443-03$ & $.17188-03$ \\
\hline $.79493-04$ & $.57016-4$ & $.37416-04$ & $.24910-04$ & - $16524=04$ \\
\hline $.16605-03$ & $.16593-3$ & $.16575-c 3$ & $.16417-03$ & $.15995-03$ \\
\hline $.88347-04$ & $.70728-04$ & .5072004 & $.33305=04$ & $.22179-04$ \\
\hline $.14289-03$ & $.14285-3$ & $.14274-03$ & $.14259-03$ & $.14123-03$ \\
\hline . $89083-04$ & $.75780-04$ & $.60587-04$ & $.43427-04$ & $.28496=04$ \\
\hline $.13372-05$ & $.1163-3$ & $.11626-03$ & $.11617-03$ & $.11604-03$ \\
\hline $.83599-04$ & $.73073-04$ & $.61512-04$ & $.48843-34$ & $.34838-04$ \\
\hline $.26969-05$ & $.11403-5$ & $.04931-04$ & . $84903-04$ & $.8484 i-04$ \\
\hline $.67848-04$ & $.01036-04$ & $.53334-04$ & $.44863=04$ & $.35421=04$ \\
\hline $.37988-05$ & $.22500-5$ & $.958 .19-06$ & .571 & $.57081-04$ \\
\hline $.49513-04$ & $.45617-04$ & $.41033-04$ & . $35871-04$ & $.30147-04$ \\
\hline $.47298-05$ & $.31111-5$ & $.186,14-05$ & $.79001-06$ & $.38822-04$ \\
\hline $.35803-04$ & $.33668-04$ & $.31016-04$ & $.27913-04$ & $.24409-04$ \\
\hline $.53339-05$ & $.37336-05$ & $.24764-05$ & $.14990-05$ & $.63786-06$ \\
\hline $.25277-04$ & $.24210-14$ & $.22766-04$ & $.20979-04$ & $.18890=04$ \\
\hline $.54446-05$ & $.40045-35$ & $.28482-05$ & . $19187-05$ & $.1163<-05$ \\
\hline $.17380-04$ & $.16930-174$ & $.16225=04$ & - $15260-04$ & $.14067-04$ \\
\hline - $50292-05$ & $.38906-5$ & $.29074-05$ & .200 & $.14272-05$ \\
\hline $.11354-04$ & $.11247-04$ & $.1095 ; 9-04$ & $.10497-04$ & $.98750-05$ \\
\hline $.42028-05$ & .33762 & $.26348-05$ & $.20005-05$ & . $14575-05$ \\
\hline $.6767 b-05$ & $.67610-15$ & $.66975-05$ & $.65258-05$ & $.02516-05$ \\
\hline $.31010-05$ & $.25414-5$ & $.20625-05$ & $.16<74-05$ & $.12388-05$ \\
\hline $.28901-05$ & . $28691-5$ & $.23860-05$ & . $28565-05$ & $.27851-05$ \\
\hline $920-05$ & .13346 & .1101 & - $\$ 9156$ & .7060 \\
\hline ADJ & 840 & & & \\
\hline $.30709-03$ & $.30690-13$ & $.30676-03$ & $.30642-03$ & $.30344-03$ \\
\hline $.18750-03$ & $.25366-13$ & $.11499-03$ & $.70220-04$ & $.358114-04$ \\
\hline $.53776-06$ & $.30694-13$ & $.30698=03$ & $.30666-03$ & $.3003 z-03$ \\
\hline $.21736-03$ & $.18743-3$ & $.15380-03$ & $.11495-03$ & $.70195-04$ \\
\hline
\end{tabular}

$.73235-05$ -15297-05 $.22665-03$ $031527-04$ .4736904 $.72135-04$ 3 . $10046-03$ 1 $57-03$ 23257-03 $12419-03$ 2r65-03 - $14300-03$ -20582-05 15622-03 $.46933-05$ $16261-03$ $.75369-05$ $.16167-03$ $.10881-04$ $15320-03$ $.14738-04$ $.13759-03$ $.19049-04$ $.13494-03$ $23097 m 04$ . $44747-04$ $.25830-04$ $57039-04$ $24015-04$ - $38009=4$ . $0.050-04$ . $16540-04$ $16549-04$ $00311=0$ - $12679-04$ 41082-05 -91082-05 $.90908-06$ . 58826-05 $.91226-06$ $24607-05$ $.54555-06$

- $29543-03$ . $18943-04$ . 30333-03 . $358101-04$
$.68920-05$ . $11218-05$ . $21714=03$ 20897-04 .22658-03 -31517-04 .23238-03 $.47337-04$ .23436-03 72063-04 - $23241=03$ -2650-03 - $22650-03$ 12092-03 . $13673=03$ 19691-05 $.14646-03$ $047-05$ $.14902-03$ $.69196-05$ $.14409-03$ .97296-05 $.13178-03$ $.12736-04$ . $11198-03$ $.15689-04$ $.33943-04$ -18044-04 $56979-04$ $38781=04$ - $16657-04$ $.06250-04$ $.14045-04$ .14045-04 -17590-04 .37593-06 .82183-05 $.61773-06$ $.54281-05$ . 63099-06 $.39947-06$

. 28261-03 - $99324-05$ $.29533=03$
.63651-05 $.78747-06$ $.20429-03$ $13742-04$ 21707-0 $22642-03$ $31494-04$ -31494-04 $.23215-03$ .47256-04 . $71374-04$ $.71374-04$ 22634-03 -96883-04 21667-03 - $11.563-03$ -20326-03 12824-03 $.18483-05$ .13420-03 40466-05 13279-03 $.61978-05$ 12393-03 84429-05 .10725-03 $.10597-04$ $81777-04$ $12512-04$ 13685-04 38739-04 13038-04 $13038-04$ 26221-04 11480-04 - $17583-04$ 14929-05 11379-04 .72327-05 -26689-06 49016-05 38974-06 .23177-05 .27783-06

.26518-03 $.53091-05$ -28252-0 $.99275-05$
.57613-05 .50871-06 18836-03 - $87248-05$ $.20422-03$ -13738-04 .21693-03 .20876-04 .22619-03 -31462-04 . 22995003 - $46838-04$ - $22609-03$ . $69498-04$ - 21651-03 .20319-03 -10038-03 18633-03

-11748-03

-17003-05

-11956-03

-36332-05

- $11420-03$ $.54043-05$ $.70914-05$ -78322-04 $.86238=05$ .54984-04 $.97851-05$ $.38371-04$ .99889-05 .26191-04 . 91683-05 $.17570-04$ .78335-05 .61963-05 $.67761-05$ $.43248-05$ -17041-06

$.17355-06$

.24337-03 $.27942-05$ $.26509-03$ $.53088-05$
- 50895-05 $.24840-06$ 16969-03 $0830-03$ $.87216-05$ $.8216-05$ $.13729-04$ 13729-04 $.21671-03$
$.20857-04$ -20857-04 - $22406-03$ $.31164-04$ $22395-03$ $.45606-04$ . 21627-03 $66450-04$ $.20305-03$ $.86823=04$ -18627-03 -99254-04 - 16611-03 $.10462-03$ .15262-05 $.10278-03$ .31754-05 $.92918-04$ $.45691-05$ $. .73644=04$ $.56281-05$ 52659-04 $.52659=04$ 37385-04 $.37385-04$ $.74099-05$ .25945-04 71842-05 - $17553-04$ $.63208-05$ 11368-04 $.51486-05$ $.67741-05$ - $37148-05$ -28918-05 $.74801-07$

$.21744-03$ $.14115-05$ .27927-0 
$.14097-05$ - $24311-03$ - $27648-05$ $263463-03$ -52556-05 . $27920-03$ - $98090-05$ - $28408-03$ . $27601-03$ - $32808-04$ . $20296-03$ - $60490-04$ .23975-03 -89599-04

- $21110-03$

$.10543-03$

-17689-03

-10760-03

.31<65-06

.95022-04

- $66486-06$

$.06383-04$

$.10238-05$

- $13831-05$

-13178-04

- $167160-05$

-12506-04

-17154-05

-70267-05

- $14938-05$

- 38156-05

-11575-05

-19066-05

$.78529-06$

$.76404-06$

- 38415-06

- 36080-03

.23191-03

. $50838-05$

. 26254-03

- $10894=04$

31337-0

. $27587-04$

.27587-04

$.32952-03$
$.40524-04$
$.53969-6$

. $21721-15$

$.14105-5$

$24266-03$

$278 n 3-0$

$26205=$

$.52443-15$

.95357-u5

.27126-13

$.17342-14$

$.26034=13$

$30594-14$

$.54581-14$

$21094=$

$78635=$

$17603-3$

8R061-i

. $31724=4$

- $25789-6$

$52310-1-0$

$.34926-14$

$.78312-6$

列

197n-

$11063=$

$68337-5$

$.10605-75$

.37777-i?

$86468=16$

19557-: 5

$.76530-16$

$.31042-6$

$36075=3$

$.3607 r-13$

$23183-1$

$50864-05$

$26237-13$

$.10890-14$

- $28964-13$

$.17975-14$

$27330-4$
$.30678-03$ - $1.5730-03$ - $53759-06$ - $21099-03$ - 14 lina-us $.24 \pi 50-03$ $.27542-05$ $.254081-013$ - 50959-05 - $25948-03$ - $9 \operatorname{arn}>9-05$ - $25352-03$ - $15151-04$ - 23714203 . $27733-04$ $.21070-03$ $.47415-04$ - 17670-03 13466-0́3 - 6r718-04 - $0736-04$ .21141-06 - $317>9-04$ - $42549-06$ - 1 ñ970-04 - $5735 \pi-06$ - 111 द $.69200-66$ - $652>4-05$ $.70924-06$ $.36734-05$ . 19459 0E . 46906-06 $.76411-06$ .24822200 $.36050-03$ $.16420-03$ $.36065-03$ $.19800-03$ . $350151-03$ . 23167-03 - $50813-05$ - हhर10-03 - $10879-04$ $.17807-04$
$.30060-03$ - $15369-3$ - $30646-03$ $.18711-03$ - $53713-06$ .21487-0 - $13437-05$ -23400-63 $26+104=05$ $.48528-05$ $.24250-23$ - $84561-05$ . $23087-03$ - 14633-0 $464=0$ $17050-03$ - $13455-03$ - 4924400 . $41207-04$ 04350 . 435804 $.31701-6$ -4j526=0 . $45,241=06$ . $35049-05$ 43655-06 -18916-05 .34 . $.755830-06$ - $18480-06$ - $36013-13$ - $+2384-33$ $.36043-03$ $.16415=03$ - $36045-03$ $19877=03$ .36014-03 -33014-03 50751-0 25964-03 . $10775-04$
$.30444-03$ $.11487-03$ - $30036-03$ - $15353-03$ . $30347-03$ $.18528=03$ $.53166-06$ $.20906-03$ 13539-05 $.22542-03$ $.25523-05$ $.22752=03$ - $4516 \mathrm{~h}-0.5$ $76521-05$ $.20312-03$ 17477-03 1. $1390-04$
.134003 - $13440-03$ - 295nz-04 - $34001-04$ $.04341-04$ - $21415=04$ - $24161-04$ $.14374-04$ $.12240-06$ $.90412-05$ . $22405-06$ $.55653-05$ $.28359-06$ -32757-05 . $29338-06$ $.18047-05$ $.24110-06$ $.13795=06$ $.06060-04$ $.36003=03$ $.12380=03$ - $16404-03$ . $36008-03$ - $19850-03$ . $35676-03$ $.50276-05$
$30610-03$ $.70146-04$ - 3n612-03 -11475-03 . $30338-03$ . $15203-03$ (1) $18026-03$ $51681-06$
$19959-03$ 19959-03 $.20878-03$ $.23755-05$ $.20717-03$ $40884-05$ $.19427-03$ $.66751-05$ $.17014-03$ 47-04 $1.3309=03$ $.15074-04$ . $12975-04$ - $18975-04$ .4530 - $15833-04$ 1629-04 13018-0 .77209-05 $96053-07$ .49360-05 $15971-156$ $.29901-05$ Inijao-06 $.16871-1,5$ .16781-06 $70132-06$ - $10504-06$ $.34765-03$ $50774-04$

$.35661-03$ - $46030-04$ -35980- 3 12372-0 $35982-03$ 16387-03 $.35670=03$ $.19671-03$

.30312-03 $.35777-04$ - 30578-03 $.70076-04$ $.30315-03$ .11362-03 $.29520-03$ 


$.33490-03$
$.57551-04$
$.32905-03$
$.79287-04$
$.31214-03$
$110710-03$
$.28745-03$
$.13044-03$
$.25823-03$
$.14169-03$
$.22522-03$
$.14394-03$
$.31975-05$
$.13666-03$
$.58166-05$
$.11606-03$
$.79508-05$
$.89477-04$
$.97551-05$
$.67526-04$
$.10898-04$
$.49341-04$
$.11117-04$
$.34833-04$
$.10363-04$
$.23365-04$
$.87854-05$
$.14427-04$
$.66745-05$
$.68567-05$
$.38000-05$

$.32106-3$

$\begin{array}{rr}.32106-03 & .30246-03 \\ .39472-04 & .26520-04\end{array}$ $.32066-03$ $.55069-0$ $.30915-13$ $.74465-44$ .28714-98327-14 . $25866-1$ $.11677-03$ $.22519-13$ $12295=$ $.18459-13$ . $12031-3$ $.27331-5$ $.10477-\hat{3}$ $48715-15$ - $82575-24$ - $65575-5$ $63552-04$ $07504-05$ $7707-1.5$ $.47276=-14$ - $33930-4$ - $33934-54$ - 2314?-14 - 70864-15 $.144 .12-14$ $.54922-15$ $.6854+4-115$ $.30740-03$ -37764-04 - 301?7-03 - 51 6h $3=04$ - $20440-03$ - $6.9280-04$ - 2577800 $.87957-04$ - $10192-03$ - $19856-03$ -10253-03 $.14472-03$ - 92152-04 $.23512-05$ . 74502-04 $.40476-05$ . $58629-54$ .52285-05 - $44488-04$ - 50505-05 - 32519-04 - $60767-05$ - $25500-04$ -54599-05 - $1+277-04$ - $44774-05$ $.26419-05$
$.27956-03$ - $17343-04$ - $28955-03$ - $25467-04$ - $28880-03$ .3543304 . $27713-03$ $.47410-04$ . $2553<-03$ - $61050-04$ $.22480-03$ . 76041-04 $.18043-03$ - $3533-04$ $.14469-03$ $.78375-04$ . $10303-03$ $.65475-04$ - 1954750505 - $52804-04$ - $32686-05$ - $32680-05$ - $40040-05$ - $40820=0$. $.33090-05$ . $1605-04$ - $21605-j 4$ - $42543=05$ - $13910-04$ - 35460-05 . $21481-05$
$.25296-03$ -10495-04 .26764-03 $16594-04$ -27204-03 - $23903-04$ - $26565-03$ . 32527-04 -24878-03 $0.370=04$ $22264-03$ $.52640=04$ -18823-03 - hack9-04 . $14459-03$ - $53246-04$ $555,3=04$ $.73181=04$ $.46489-04$ $13786-0.5$ $.25472-05$ $0.30+30-05$

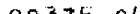
. $31213-05$ $.13328-04$ . $27098-05$ $.17122-05$
MESH BOUNDARIES (RI/ZI=RDOIAL DOINTS/AXIAL POINTS

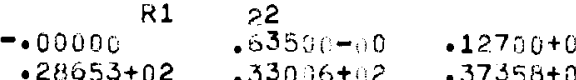
R1

$.190500+01$

$.25400+01$

$\begin{array}{rrrr}.63506-00 & .12700+01 & .19050+01 & .25400+01 \\ .33721+12 & .34175+02 & .41965+02 & .45795+02\end{array}$

$.57225+02$
$22735-03$ $.48984-05$ $.24213-03$ $120046-0$ $.25143-03$ $.15583-04$ $.25019-03$ . $21957-04$ 2.3845-03 29094-04 $.21694-03$ 36597-04 18643-03 $.43255-04$ $.14444-03$ $.47811-04$ 10292-0 3 14 $4877-04$ 73162-04 - 3943204 51236-04 51236-04 32 $555-04$ - $17282-05$ - $25500-04$ - $19290-05$ - $18776-04$ . 21672-05 - $12550-04$ -201112-05 $.13305-05$

$.19161-03$ .21379-03 . $22743-03$ -94332-05 .23119-03 -14322-04 .22456-03 $.19675-04$ $.20791-03$ .25250-04 . 18165-03 $.30490-04$ $.14306-03$ $.34447-04$ -10282-03 $.35109-04$ .73 $111-04$ .51212-04 - $51221-04$ -27766-04 - 245004 - $22458-04$ - $92250-06$ -16965-04 -13834-05 -11588-04 -14119-05 $.97951-06$

$.68922+01$

$.11244+02$ $.53140+02$ $.56950+02$

$.19949+02$

$.0 .0357+02$ $.61035+02$

$.82865-04$ $.33220-03$ .11411-03 -31247-03 -14205-0.3 -28764-03 .15817-03 -258? 7-03 . 16542-03 .36446-05 .16335-03 .683,5-05 .15132-03 $.95272-05$ -12572-03 .11938-04 . $95071-04$ - $13900-04$ -13990-04 -70482-04 $.14891-04$ 04 35171-04 -35171-04 - 12929-04 -23393-04 - $10718-04$ $.14441-04$ -79730-05 . 68594-05 $.44061-05$
$.21692-06$

$.24301+02$ $.64570+02$

$.69944+01$
$.49605+02$

$.11449+02$

$15903+02$

$.24812+02$

$.64845+02$

0

\begin{abstract}
.
\end{abstract}
$\begin{array}{ll}2 & 2 \\ 3 & 3 \\ 2 & 2 \\ 3 & 3 \\ 2 & 2\end{array}$

2
3
2
3
2



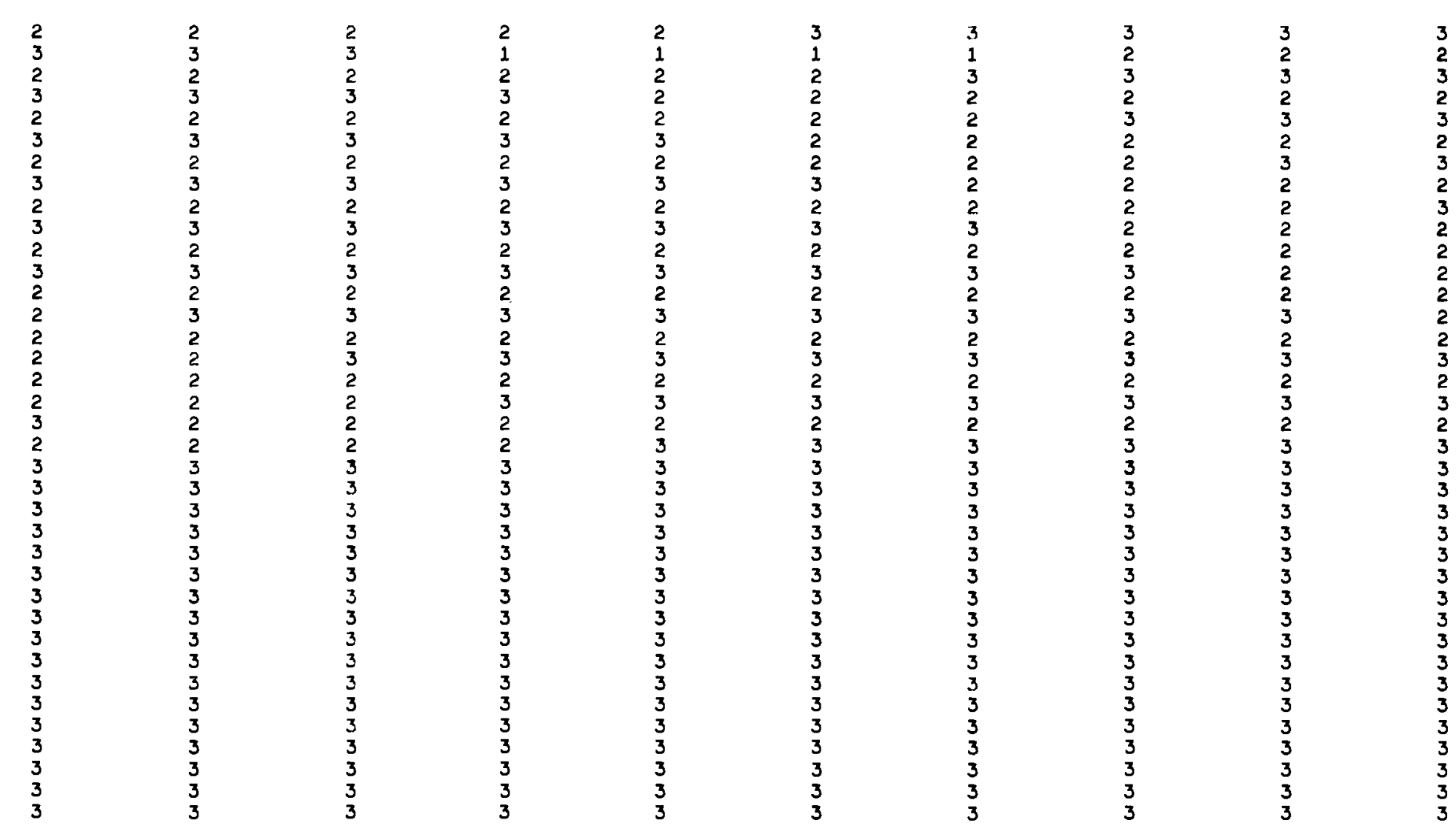

MATERIAL NUMBERS BY ZONE $\begin{array}{lll}\text { M2 } & 3 & \\ 19 & & 17\end{array}$

FISSION SPECTRUNA

$\begin{array}{cc}\text { CHI } & 2 \\ .98700-00 & .13000-01\end{array}$

MIXTURE SPECIFICATIONS $(I 0 / I 1 / I 2=M I X$ NUMPER/MAT. NUMBER FOR MIX/MATERIAL DENSITY)

$\begin{array}{llllllll}10 & 31 & 17 & 17 & 17 & 17 & 17 & 17\end{array}$

17

17

17

17

$w$
2
$\vdots$
1
0
0
0 


\begin{tabular}{|c|c|c|c|c|c|c|c|c|c|}
\hline 17 & 17 & 18 & 18 & 18 & 18 & 18 & 18 & 19 & 19 \\
\hline 19 & 19 & 19 & 19 & 19 & 19 & 19 & 19 & 19 & 19 \\
\hline I1 & 31 & & & & & & & & \\
\hline 0 & 1 & 2 & 3 & 4 & 5 & 6 & .7 & 8 & 9 \\
\hline 10 & 11 & 0 & 12 & 13 & 14 & 15 & 16 & 0 & 1 \\
\hline 2 & 3 & 4 & 5 & 6 & 7 & 8 & 9 & 10 & 11 \\
\hline I2 & 31 & & & & & & & & \\
\hline .00000 & $.1654 \pi-02$ & .10600003 & $.16000-04$ & $.74660-02$ & $.20765-01$ & $.62300-02$ & $.98990-02$ & $.26580-02$ & $.13020-02$ \\
\hline . $266000-03$ & $.11000,03$ & .00000 & $.04000-04$ & $.39976-01$ & $.44530-02$ & $.11960-02$ & $.58800-03$ & .00000 & $.16540-0 ?$ \\
\hline$-10600-03$ & $.16000-04$ & $.74060-02$ & $.20765-01$ & $.62300-02$ & $.98990-02$ & $.26580-02$ & $.13080-02$ & $.20600-03$ & $.11000=03$ \\
\hline
\end{tabular}

MATERIAL NUMBERS FOR BETA CALCULATIOR:
NABET
$\begin{array}{ll}6 & 2 \\ 6 & 2\end{array}$
3
4
12
13

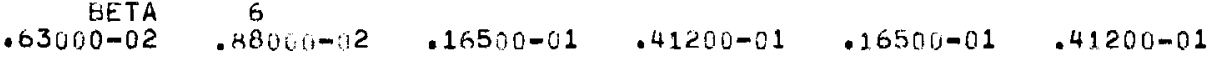

DELAYED FISSION SPECTRURA

$.10000+01 \quad .0000 \%$

NEUTRON VELOCITY

$.76309+09 \quad .11636+19$

PERTURBATION CALCULATTON INFORINATION (MATUK/NROW/NCQL=WATERIAL NUMBER/ROW/COLUMN TO BE PERTURBED)

$\begin{array}{rrrr}\text { MATDK } & 3 & 1 & 6 \\ 1 & 3 & 1 & 1 \\ \text { INROW } & 3 & 0 & 0 \\ \text { NCOL } & 3 & 5 & \end{array}$

ACTIVITY TRAVERSE ITFORMATION (MAM/WX EMATERIAL NUMGER/CROSS SECTION POSITION)

$\begin{array}{cc}M A & 1 \\ 1 & \\ N X & 1 \\ 1 & \end{array}$




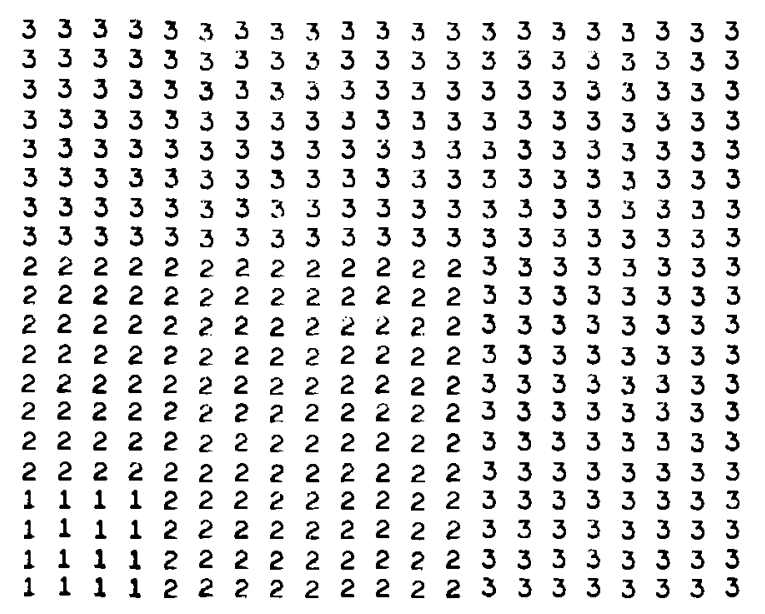

RADIAL 
$18181818181 \% 181813181816181818181818181818$ 181818181818181815161818181818181818181818 181818181818181418161818181818181818181618 18181818181 a131813161818181818181018131618 $181818181816181 B 16181818181818181018181818$ 1818181818191816181618 A81\&1818181818181818 181818181818181813131818181818181818181818 181618181818181818181818181818181018181818 171717171717171717171717171818181018181818 171717171717171717171717171818181818181818 171717171717171717171717171818181818161818 171717171717171717171717171818181618181818 171717171717171717171717171810181818181818 171717171717171717171717171818181818181818 171717171717171717171717171818181818181818 18181 17171717177171717717717181818181828131

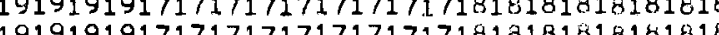
191919191717171717171717171810181018101818

191919191717171717171717171818181018181818

RADIAL 
CROSS-SECTION EUIT

\section{GROUP 1 CROSS-SECTIONS}

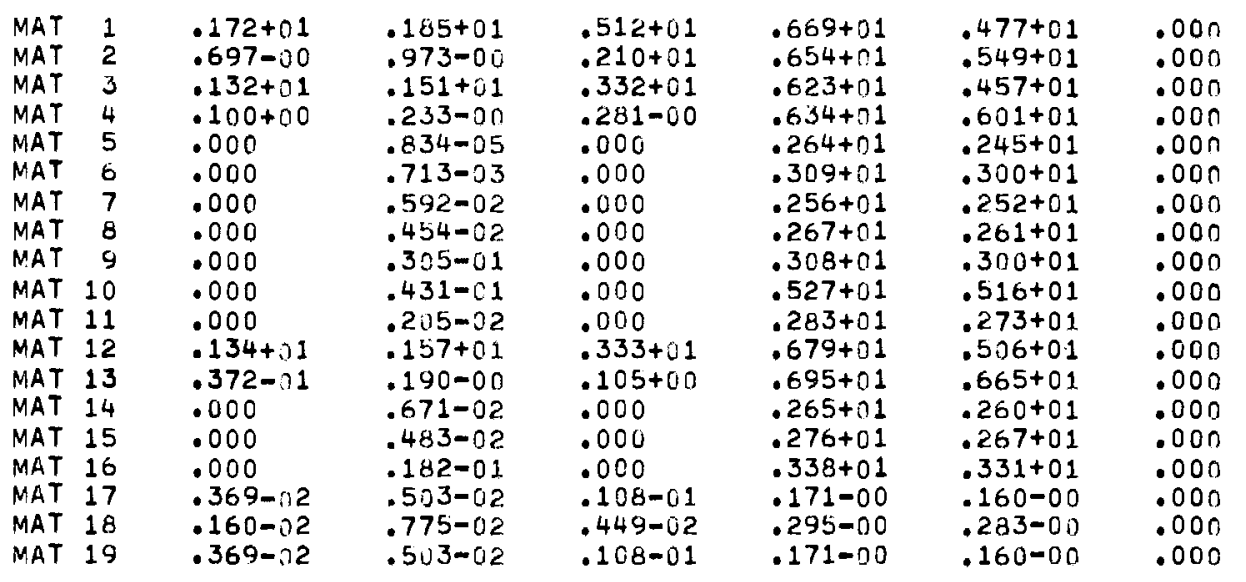

\section{GROUP 2 CROSS-SECTIINS}

$\begin{array}{llll}\text { MAT } & 1 & .228+01 & .324+01 \\ \text { MAT } & 2 & .205-01 & .158+01 \\ \text { MAT } & 3 & .336+01 & .487+01 \\ \text { MAT } & 4 & .000 & .532-00 \\ \text { MAT } & 5 & .000 & .457-10 \\ \text { MAT } & 6 & .000 & .423-02 \\ \text { MAT } & 7 & .000 & .215-01 \\ \text { MAT } & 8 & .000 & .150-01 \\ \text { MAT } & 9 & .000 & .204-01 \\ \text { MAT } 10 & .000 & .303-00 \\ \text { MAT } 11 & .000 & .830-02 \\ \text { MAT } 12 & .212+01 & .284+01 \\ \text { MAT } 13 & .000 & .405-00 \\ \text { MAT } 14 & .000 & .120-01 \\ \text { MAT } 15 & .000 & .024-02\end{array}$

$\begin{array}{llll}.650+01 & .139+02 & .107+02 & .790-01 \\ .578-01 & .139+02 & .124+02 & .749-01 \\ .815+01 & .147+02 & .985+01 & .147-00 \\ .000 & .132+02 & .126+02 & .976-01 \\ .000 & .449+01 & .449+01 & .188-00 \\ .000 & .498+01 & .498+01 & .890-01 \\ .000 & .482+01 & .480+01 & .342-01 \\ .000 & .472+01 & .471+01 & .596-01 \\ .000 & .945+01 & .943+01 & .424-01 \\ .000 & .789+01 & .759+01 & .705-01 \\ .000 & .217+01 & .216+01 & .968-01 \\ .515+01 & .123+02 & .948+01 & .168-00 \\ .000 & .122+02 & .118+02 & .112+00 \\ .000 & .450+01 & .449+01 & .450-01 \\ .000 & .441+01 & .440+01 & .791-01\end{array}$


$\begin{array}{lllllll}\text { MAT } 16 & .000 & .169-01 & .000 & .750+01 & .748+01 & .545-01 \\ \text { MAT } 17 & .383-02 & .992-02 & .109-01 & .321-00 & .311-00 & .591-02 \\ \text { MAT } 18 & .178-03 & .165-01 & .433-03 & .519-00 & .502-00 & .483-02 \\ \text { MAT } 19 & .383-02 & .992-02 & .109-01 & .321-00 & .311-00 & .591-02\end{array}$ 
MIXTURE NUMBER

$\begin{array}{ll}1 & 17 \\ 2 & 17 \\ 3 & 17 \\ 4 & 17 \\ 5 & 17 \\ 6 & 17 \\ 7 & 17 \\ 8 & 17 \\ 9 & 17 \\ 10 & 17 \\ 11 & 17 \\ 12 & 17 \\ 13 & 18 \\ 14 & 18 \\ 15 & 18 \\ 16 & 18 \\ 17 & 18 \\ 18 & 18 \\ 19 & 19 \\ 20 & 19 \\ 21 & 19 \\ 22 & 19 \\ 23 & 19 \\ 24 & 19 \\ 25 & 19 \\ 26 & 19 \\ 27 & 19 \\ 28 & 19 \\ 29 & 19 \\ 30 & 19 \\ 31 & 19\end{array}$

MIX COMMAND

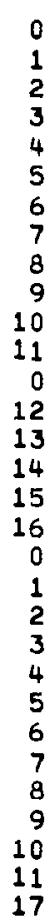

NATERIAL ATOMIC DENSITY

$$
\begin{aligned}
& .00000000 \\
& .16540000-02 \\
& .10600000-03 \\
& .16000000-04 \\
& .74060000-02 \\
& .20764999-01 \\
& .62300000-02 \\
& .98989999-02 \\
& .26579999-02 \\
& .13080000-02 \\
& .20599999-03 \\
& .11000000-03 \\
& .00000000 \\
& .83999999-04 \\
& .39975999-01 \\
& .44529999-02 \\
& .11960000-02 \\
& .58799999-03 \\
& .00000000 \\
& .16540000-02 \\
& .10600000-03 \\
& .16000000-04 \\
& .74060000-02 \\
& .20764999-01 \\
& .62300000-02 \\
& .98989999-02 \\
& .26579999-02 \\
& .13080000-02 \\
& .20599999-03 \\
& .11000000-03 \\
& .99999999-10
\end{aligned}
$$


PERT-IV TEST CASE

RADIT

-.0000
.6350
1.2700
1.9050
2.5400
6.8922
11.2444
15.5967
19.9489
24.3011
28.6533
33.0056
37.3578
41.7100
45.5200
49.3300
53.1400
56.9500
60.7600
64.5700
68.3800
72.1900
(2DF FLLIX INPINT, 2 GROUPS, 3 ZONES)

AVG RADII

.3175
.9525
1.5875
2.2225
4.7161
9.0683
3.4206
7.7728
2.1250
6.4772
0.8294
5.1817
9.5339
3.6150
7.4250
1.2350
5.0450
8.8550
2.6650
6.4750
70.2850
.0000

AXI I

$-.0000$

.6350
1.2700

1.9050

2.5400
6.9944

11.4487

15.9031

20.3575

24.8119

29.2662

33.7206

38.1750

41.9850

45.7950

49.6050

53.4150

57.2250

61.0350

64.8450

68.6550

.0000
Avg AXII

.3175
.9525
1.5875
2.2225
4.7672
9.2216
13.6759
18.1303
22.5847
27.0391
31.4934
35.9478
40.0800
43.8900
47.7000
51.5100
55.3200
59.1300
62.9400
66.7500
.0000
.0000


MATERIAL INVENTORY (KILOGRAMS) FOR EACH ZONE

\begin{tabular}{|c|c|c|c|c|}
\hline MATERIAL & ATOMIC NT. & $\begin{array}{l}\text { ZONE } 1 \\
-10 \text { LITERS }\end{array}$ & $\begin{array}{c}\text { ZONE }{ }^{2} \\
417.19 \text { LITERS }\end{array}$ & $\begin{array}{c}\text { ZONE } 3 \\
183 n .76 \text { LITERS }\end{array}$ \\
\hline 1 N.2PU49 & 239.150 & .068 & 275.869 & .000 \\
\hline 2 PU240 & 240.050 & .004 & 17.625 & .000 \\
\hline 3 M2U235 & 235.040 & .001 & 2.605 & .000 \\
\hline $4 \cup 238$ & 238.050 & .301 & 1221.155 & .000 \\
\hline $5 c$ & 12.011 & .043 & 172.755 & .000 \\
\hline 6 NA & 22.990 & .024 & 99.206 & .000 \\
\hline $7 \mathrm{M} 2 \mathrm{FE}$ & 55.847 & .095 & 382.922 & .000 \\
\hline B CR & 51.996 & .024 & 95.729 & .000 \\
\hline $9 \mathrm{NI}$ & 58.710 & .013 & 53.191 & .000 \\
\hline $0 \mathrm{MO}$ & 95.940 & .003 & 13.689 & .000 \\
\hline $1 \mathrm{AL}$ & 26.981 & .001 & 2.056 & .000 \\
\hline 2 M2U235 & 235.040 & .000 & .000 & 60.012 \\
\hline $3 \cup 238$ & 238.050 & .000 & .000 & 24925.792 \\
\hline $4 \mathrm{M} 2 \mathrm{FE}$ & 55.847 & .000 & .000 & 755.910 \\
\hline $5 \mathrm{CR}$ & 51.996 & .000 & .000 & 189.025 \\
\hline $6 \mathrm{NI}$ & 58.710 & .000 & .000 & 104.932 \\
\hline
\end{tabular}




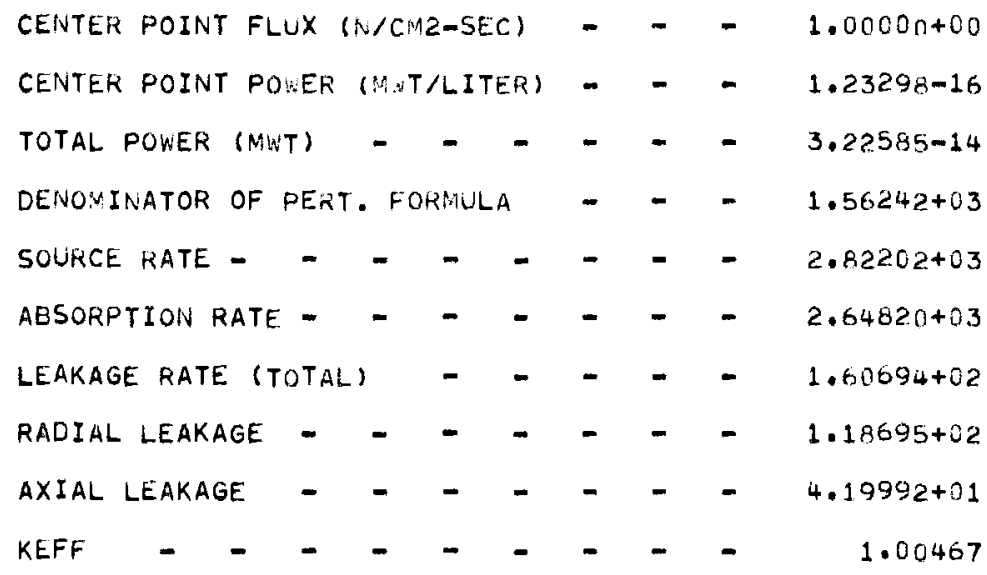


TOTAL FLUX

$\begin{array}{rr} & \\ 1 & .1000000+01 \\ 2 & .9996795-00 \\ 3 & .9991206-00 \\ 4 & .9980810-00 \\ 5 & .9886532-00 \\ 6 & .9630553-00 \\ 7 & .9215870-00 \\ 8 & .8649799-00 \\ 9 & .7941176-00 \\ 10 & .7100870-00 \\ 11 & .6142483-00 \\ 12 & .5049654-00 \\ 13 & .3740842-00 \\ 14 & .2557237-00 \\ 15 & .1768079-00 \\ 16 & .1212360+00 \\ 17 & .8213459-01 \\ 18 & .5386170-01 \\ 19 & .3278025-01 \\ 20 & .1508522-01 \\ & \\ 1 & .9632435-00 \\ 2 & .9629262-00 \\ 3 & .9622554-00 \\ 4 & .9612813-00 \\ 5 & .9522604-00 \\ 6 & .9275754-00 \\ 7 & .8876345-00 \\ 8 & .8330732-00 \\ 9 & .7647834-00 \\ 10 & .6838216-00 \\ 11 & .5915143-00 \\ 12 & .4862562-00 \\ 13 & .3602213-00 \\ 14 & .2462491-00 \\ 15 & .1702648-00 \\ 16 & .1167406+00 \\ 17 & .7909443-01 \\ & \end{array}$

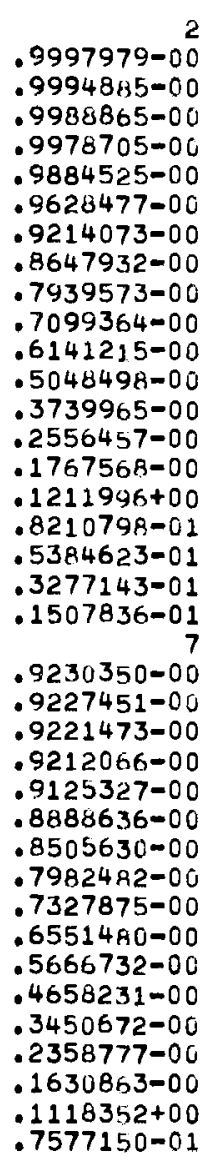

3
$.9990830-00$
$.9988517-00$
$.9981660-00$
$.9971487-00$
$.9877933-00$
$.9621774-00$
$.9207746-00$
$.8642093-00$
$.7934054-00$
$.7094487-00$
$.6136992-00$
$.5045000-00$
$.3737378-00$
$.2554643-00$
$.1766346-00$
$.1211076+00$
$.8204861-01$
$.5381417-01$
$.3273916-01$
$.1507447-01$
$.8689002-00$
$.8685908-00$
$.8680069-00$
$.8671595-00$
$.8589985-00$
$.8366854-00$
$.8006229-00$
$.7513225-00$
$.6896176-00$
$.6165115-00$
$.5331930-00$
$.4382421-00$
$.3246087-00$
$.2218843-00$
$.1534238-00$
$.1052000+00$
$.7128031-01$

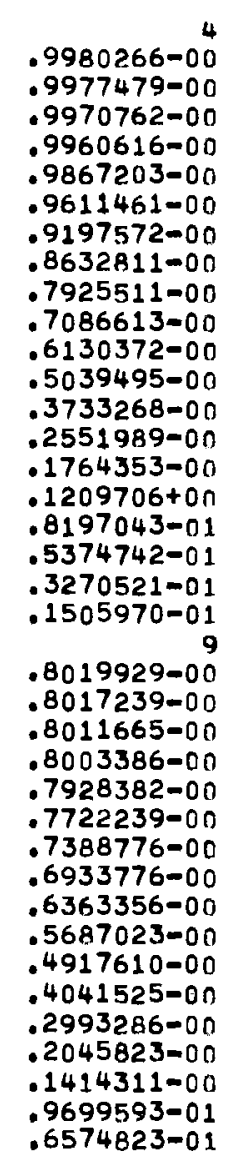

.9885766-0 5 $.9885766-00$
$.9882198-00$ $.9882198-00$
$.9875564-00$ .9875564-00 .9773106-00 . 9519887-00 . 9110012-00 . 8550156-00 .7849783-00 .7018953-00 $.6071506-00$ $.4991318-00$ .3697648-00 $.2527595-00$ $.1747561=00$ $.1747561=00$ - $1198328+00$ $.8118136-01$ - $3323652-01$ - 3239885-01 .1491372-0

$.7238461-00$ $.7236064-00$ $.7231326-00$ .7223925-00 $.7155625-00$ $.6969577-00$ $.6667833-00$ $.6256313-00$ $.5741373-00$ .5129564-00 $.4433232-00$ .3642139-00 .2696928-00 - $1842821=00$ $.1274341=00$ .8742753-01 5931518-01 


$\begin{array}{rr}18 & .5187190-01 \\ 19 & .3156643-01 \\ 20 & .1453031-01 \\ 1 & .6361837-00 \\ 2 & .6359663-00 \\ 3 & .6355343-00 \\ 4 & .6349126-00 \\ 5 & .6289021-00 \\ 6 & .6124993-00 \\ 7 & .5859691-00 \\ 8 & .5496544-00 \\ 9 & .5042861-00 \\ 10 & .4503637-00 \\ 11 & .3899489-00 \\ 12 & .3193492-00 \\ 13 & .2363606-00 \\ 14 & .1614953-00 \\ 15 & .1116493+00 \\ 16 & .7670968-01 \\ 17 & .5216265-01 \\ 18 & .3431639-01 \\ 19 & .2096513-01 \\ 20 & .9669658-02 \\ & .1439374-00 \\ 1 & .1436 \\ 2 & .1438986-00 \\ 3 & .1437843-00 \\ 4 & .1436436-00 \\ 5 & .1422893-00 \\ 6 & .1385570-00 \\ 7 & .1325453-00 \\ 8 & .1242794+00 \\ 9 & .1139538+00 \\ 10 & .1016233+00 \\ 11 & .8747756-01 \\ 12 & .7230852-01 \\ 13 & .5795153-01 \\ 14 & .4552272-01 \\ 15 & .3458529-01 \\ 16 & .2559708-01 \\ 17 & .1846630-01 \\ 18 & .1260488-01 \\ 19 & .7957160-02 \\ 20 & .3726299-02 \\ 1 & .1115890-01 \\ 2 & .1116591-01 \\ 3 & .1115376-01 \\ & \end{array}$

.4315451-01 2627719-01 $.1210034-01$ . 3189418-00 3188355-00 . $3186210-00$ $.3183022-00$ 3152794-00 $.3070444-00$ .2936808-00 $2936808=00$
$.2754323-00$ $.2524737-00$ - $2524737-00$ - $2251717-00$ - 1935384-0n -1565285-0n - $1177081+00$ .8446820-01 . 5034269-01 .4289293-01 .2977864-01 .2003979-01 $.1237310-01$. $.5778430-02$ $.4134772-01$ $.4134772-01$ $.4129239-01$ $.4125875-01$ 4087116-01 3980315-01 3808221-01 $.3574597-01$ - $3574597-01$ - $2283479-01$ . - 2568666-01 -2175316-01 -1811952-01 -1485646-01 -1184803-01 -9223159-02 . 6884469-02 48533A9-0? . $3134061=0$ ? $.1484617-0$ ?
- 3896344-01 2374065-01 1094963-01

.2128196-00 2127468-00 2126040-00 $2123760-0$ - $2103767-00$ - 2048719-00 -1959591-00 -1837746-00 $1684333-00$ 1502390-00 - $1289354-00$ $1050921+00$ .8282429-01 .6325743-01 4644917-01 .3373243-01 -2377770-01 -1611872-01 -1006868-01 .4685162-02

.2468115-01 2466865-01 2466053-0 -2463484-01 .2439971-01 . $376388-01$ - 2374352-01 .2135013-01 2135013-01 -1962216-01 $1743128-01$

1542624-01

-1311704-01

1096255-01

9094264-02

7329826-02

-5694604-02

4301264-02 3070191-02

$.1976217-02$
$.9507971-03$

$.606 j 430-02$

. $2892055-02$

$.4486154=0$

$.2120094-02$ 


$$
\begin{array}{rr}
4 & .1114041-01 \\
5 & .1103550-01 \\
6 & .1075131-01 \\
7 & .1026664-01 \\
8 & .9659150-02 \\
9 & .8891368-02 \\
10 & : 7987053-02 \\
11 & .7002327-02 \\
12 & .5978204-02 \\
13 & .5028931=02 \\
14 & .4152796-02 \\
15 & .3368065-02 \\
16 & .2668762-02 \\
17 & .1996130-02 \\
18 & .1426049-02 \\
19 & .9329756-03 \\
20 & .4505314-03
\end{array}
$$


FISSION SOURCE*ADJOTNT FLUX

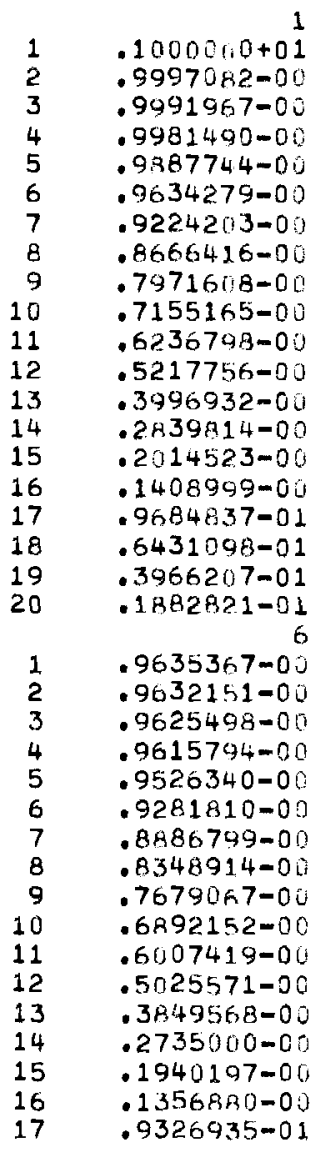

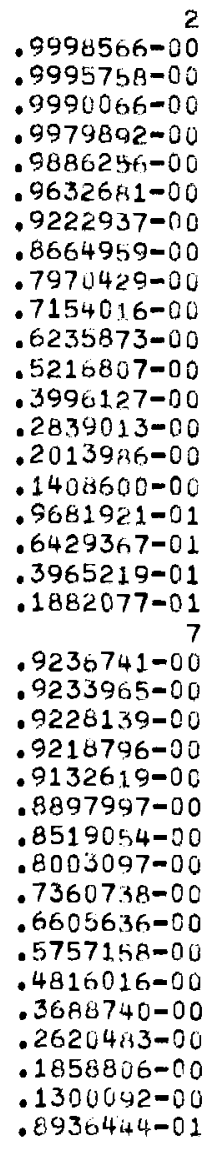

3
$.9991575-00$
$.9989677-00$
$.9982992-00$
$.9972818-00$
$.9879953-00$
$.9626160=00$
$.9216832-00$
$.8659325=00$
$.7965046-00$
$.7149272-00$
$.6231714-00$
$.5213290=00$
$.3993418-00$
$.2836999-00$
$.2012590-00$
$.1407549-00$
$.9674938-01$
$.6425497-01$
$.3961450-01$
$.1881459-01$
$.8701791-00$
$.8698687-00$
$.8692865-00$
$.8684544-00$
$.8603525-00$
$.8382088-00$
$.8025043-00$
$.7538403-00$
$.6932179-00$
$.6220560=00$
$.5420802-00$
$.4533828-00$
$.3472105-00$
$.2466231-00$
$.1749370-00$
$.1223389+00$
$.8409075-01$

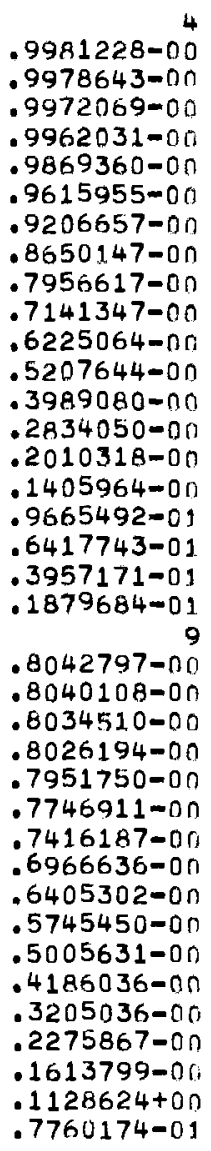

5

$.9887265-00$

- $9883608-00$

- $9876982=00$

-9867193-00

- $9775462-00$

$.9524663-00$

- $9119304-00$

$.8567460-00$

.7880757-00

-7073398-00

$.6165375-00$

.5157970-00

.3951069-00

.2807031-00

- 1991213-00

-1392727-00

-9572610-01

$.6356540-01$

.3920098-01

.1861417-01

$.7276684=00$ $.7274299=00$

$.7269614=00$

$.7269614-00$

$.7262159-00$

$.7008756-00$

. $6708557-00$

$.6708557-00$

.

.5793174-00

$.4522364-00$

- $4522364-00$

-

- $2893081-00$

- 2053196-00

-1455844-00

$.1018320+00$
$.7007153-01$ 


\begin{tabular}{|c|c|}
\hline $\begin{array}{l}18 \\
19 \\
20\end{array}$ & $\begin{array}{l}6193794-01 \\
.3819477-01 \\
.1813611-01\end{array}$ \\
\hline 1 & $.6423965=$ \\
\hline$=$ & $.6421776=0$ \\
\hline 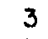 & $.6417480=0$ \\
\hline 4 & $.6411326-0$ \\
\hline 5 & $.6350998-$ \\
\hline 6 & .6186818 \\
\hline 7 & $.5921705-0$ \\
\hline 8 & $.5559800-c$ \\
\hline 9 & $.5110376-0$ \\
\hline 10 & $.4580285=0$ \\
\hline 11 & $.3984012-$ \\
\hline 12 & $.3327061-$ \\
\hline 13 & .2544077 \\
\hline 14 & $.1804157-$ \\
\hline & .1278303 \\
\hline 16 & $.8950693=0$ \\
\hline 17 & $.6170773-0$ \\
\hline 18 & $.4107843-0$ \\
\hline 19 & $.2542017-0$ \\
\hline 20 & $.1209317=0$ \\
\hline & .1645175 \\
\hline 2 & - $1644616=0$ \\
\hline 3 & $.1643443-0$ \\
\hline 4 & $.1641841-0$ \\
\hline 5 & - $1626413=0$ \\
\hline 6 & $.1583963-c$ \\
\hline 7 & $.1515628-c$ \\
\hline 8 & $.1421856=0$ \\
\hline 9 & $.1304953=0$ \\
\hline 10 & $.1165911+0$ \\
\hline 11 & $.1006870+C$ \\
\hline 12 & $.8364575-0$ \\
\hline 13 & $.6751579-0$ \\
\hline 14 & $.5343499-0$ \\
\hline 15 & $.4091229-0$ \\
\hline 16 & $.3053263-0$ \\
\hline 17 & $.2218455-0$ \\
\hline 18 & $.152611 .9-0$ \\
\hline 19 & $.9731747-0$ \\
\hline 20 & $.4697828-0$ \\
\hline & \\
\hline 1 & - $13953,38-0$ \\
\hline 2 & .1396063 \\
\hline 3 & .1394663 \\
\hline
\end{tabular}

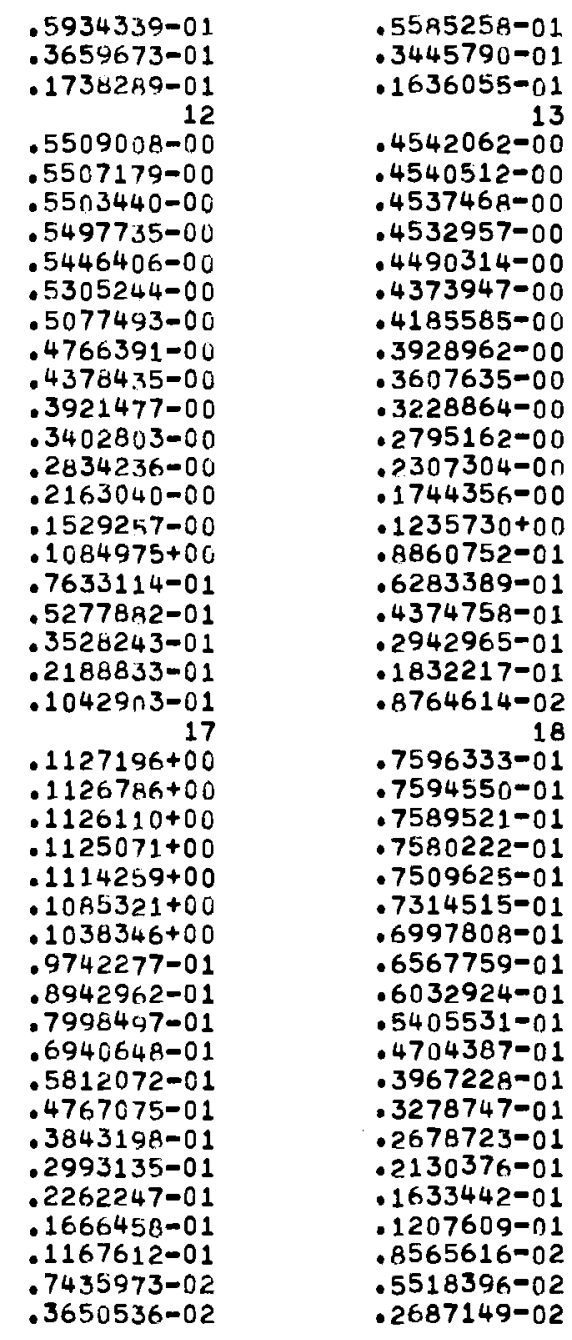

5157118-0
$.3181697-01$

.1511202-01

.3419633-00

.3418500-0n

.3416208-0n

$.3412811-00$

.3380629-00

3292923-00

.3150896-0

.2428047-0n

2098494-0n

$1717388-0 n$

-1317286-00

-9662477-01

.7024148-01

-5060665-01

3551077-01

$.2412146-01$

7250180-0?

19
-01

4947530-01

.4945305-01

$.4940987-09$

4890640-01

$.4763207-01$

$.4557920-01$

.4279742-01

3933367-01

3528023-01

$3083228-01$

.2615945-01

2103905-01

1794243-01

-1794243-01

.1435142-01

-1120330-01

. 8391491-02

3973665-02

$.3873665-02$
$.1888795-02$
.4659590-01

.2876048-01

.1368294-01

.2371703-00

. $2370899-00$

.2369303-00

.2366796-00

.2344624-00

.2283628-00

$.2184994-00$

.2050385-00

. $1881486-00$

1682050-00

1449993-00

-1449993-00

$: 1190996+00$

$.9479888-01$

$.7325826-01$

. $4550085-01$

.4000640-01

-2845693-01

-1945406-01

-1228887-01

.5894209-02

.2992415-0

-2990956-01

-2989837-01

.2986840-01

.2958365-01

.2881449-01

.2758152-01

.2589831-01

.2381155-01

$.2141107-01$

-1875368-01

.1596663-01

.1337088-01

-1110899-01

$.1110899-01$

6970361-02

.

:379567-02

.2465255-02

.2165255-02 


$$
\begin{array}{rr}
4 & .1392956-01 \\
5 & .1379967-01 \\
6 & : 1344455-01 \\
7 & : 1286480-01 \\
8 & .1208266-01 \\
9 & .1112755-01 \\
10 & .1000244-01 \\
11 & .8775192-02 \\
12 & .7500386-02 \\
13 & .6314985-02 \\
14 & .5228608-02 \\
15 & .4248862-02 \\
16 & .3369931-02 \\
17 & .2530729-02 \\
18 & .1814705-02 \\
19 & .1195761-02 \\
20 & .5949106-03
\end{array}
$$


POWER DENSITY (MWT/LITER)

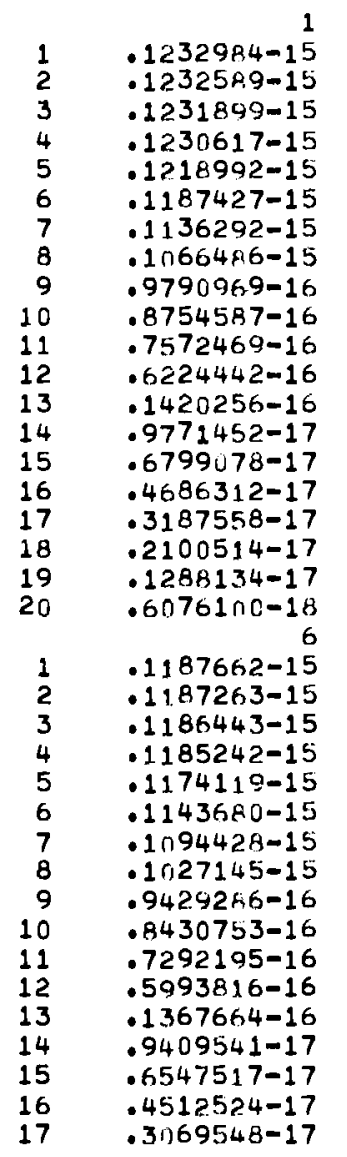

.12
$.1232733-15$
$.1232351-15$
$.1231608-15$
$.1230356-15$
$.1218743-15$
$.1187170-15$
$.1136069-15$
$.1066254-15$
$.9788979-16$
$.8752719-16$
$.7570896-16$
$.6223008-16$
$.1419976-16$
$.9768704-17$
$.6797260-17$
$.4684999-17$
$.3186571-17$
$.2099966-17$
$.1287801-17$
$.6073578-18$
$.1138082-15$
$.1137724-15$
$.1136987-15$
$.1135827-15$
$.1125132-15$
$.1095945-15$
$.1048716-15$
$.9842040-16$
$.9034769-16$
$.8077217-16$
$.6985934-16$
$.5741933-16$
$.1310224-16$
$.9013848-17$
$.6271844-17$
$.4323204-17$
$.2940757-17$

3
$.1231851-15$
$.1231565-15$
$.1230720-15$
$.1229465-15$
$.1217930-15$
$.1186343-15$
$.1135288-15$
$.1065534-15$
$.9782170-16$
$.8746701-16$
$.7565686-16$
$.6218693-16$
$.1419013-16$
$.9761882-17$
$.6792591-17$
$.4681436-17$
$.3184292-17$
$.2098737-17$
$.1286506-17$
$.6072192-18$
$.1071327-15$
$.1071327-15$
$.1070946-15$
$.1070226-15$
$.1069181-15$
$.1059118-15$
$.1031604-15$
$.9871346-16$
$.9263401-16$
$.8502456-16$
$.7600823-16$
$.6573149-16$
$.5401924-16$
$.1232769-16$
$.8480549-17$
$.5901166-17$
$.4067202-17$
$.2766679-17$
$1230548-15$ .1230204-15 .1229376-15 1228125-15 -1216606-15 -1185071-15 -1134034-15 -1064389-15 1064389-15 . $8771635-16$ $.8736993-16$ -7557525-16 $.6211907-16$ -1417456-16 -9751752-17 $.6784838-17$ .4676144-17 -3181281-17 .2096107-17 $1285155-17$ .6066417-18
9888191-16 .9884875-16 -9878003-16 .9867796-16 $.9775310-16$ .9521122-16 $.9109932-16$ $.8548854-16$ $.7845425-16$
$7011308-16$
6062308-16
$.6062308-16$
137160-16
-7137160-16
-7821566-17
$.3750757-17$
$.2552480-17$




$\begin{array}{rr}18 & .2022911-17 \\ 19 & .1240395-17 \\ 20 & .5852792-18 \\ & 11 \\ 1 & .7843313-16 \\ 2 & .7840632-16 \\ 3 & .7835305-16 \\ 4 & .7827641-16 \\ 5 & .7753533-16 \\ 6 & .7551290-16 \\ 7 & .7224175-16 \\ 8 & .6776403-16 \\ 9 & .6216902-16 \\ 10 & .5551907-16 \\ 11 & .4794573-16 \\ 12 & .3936034-16 \\ 13 & .8995271-17 \\ 14 & .6183332-17 \\ 15 & .4300383-17 \\ 16 & .2969120-17 \\ 17 & .2026597-17 \\ 18 & .1339401-17 \\ 19 & .8244304-18 \\ 20 & .3897777-18 \\ & .35 \\ 1 & .5540133-17 \\ 2 & .5538244-17 \\ 3 & .5534236-17 \\ 4 & .5528901-17 \\ 5 & .5476824-17 \\ 6 & .5333433-17 \\ 7 & .5102651-17 \\ 8 & .4785300-17 \\ 9 & .4389347-17 \\ 10 & .3916703-17 \\ 11 & .3374265-17 \\ 12 & .2792875-17 \\ 13 & .2242392-17 \\ 14 & .1764329-17 \\ 15 & .1342007-17 \\ 16 & .9958270-18 \\ 17 & .7208500-18 \\ 18 & .4934121-18 \\ 19 & .3138265-18 \\ 20 & .1506328-18 \\ & .479 \\ 1 & .4495929-18 \\ 2 & .4499207-18 \\ 3 & .4494437-18\end{array}$

$.1937979-17$
$.1198418-17$
$.5609372-18$
12
$.6670167-16$
$.6667916-16$
$.6663397-16$
$.6656456-16$
$.6593778-16$
$.6421465-16$
$.6142844-16$
$.5761451-16$
$.5283399-16$
$.4715620-16$
$.4064424-16$
$.3329980-16$
$.7614191-17$
$.5222242-17$
$.3640271-17$
$.2526996-17$
$.1730925-17$
$.1149268-17$
$.7092929-18$
$.3359361-18$
$.3740779-17$
$.3739395-17$
$.3737226-17$
$.3733823-17$
$.3697740-17$
$.3601572-17$
$.3445197-17$
$.3231637-17$
$.2965082-17$
$.2649197-17$
$.2294749-17$
$.1916747-17$
$.1567985-17$
$.1259579-17$
$.9761131-18$
$.7342181-18$
$.5393811-18$
$.3769844-18$
$.2391171-18$
$.1170665-18$

.1823741-17 -1118840-17

.5400543-16 $.5398722-16$ $.5395080-16$ $.5389669-16$ $5338581-16$ $.5338581-16$ $.5199132-16$ $.4973034-16$ $.4664155-16$ - $4275891=16$ - $3814497-16$ -3281895-16 -2669195-16 -6084869-17 $.4194896-17$ $.2961184-17$ -2074372-17 -1432085-17 . $9572611-18$ -5931295-18 $.282070 A-18$

$.2495257-17$ .2494741-17 $.2493056-17$ $.2489881-17$ .2466751-17 $.2402561-17$ -2298266-17 -2298266-17 - $1900202-17$ -1772789-17 - 1772789-17 -1540983-17 - $1297390-17$ - $1069596-17$ - 8720217-18 -6920364-18 . 5286848-18 -3894813-18 - $2758675-18$ - 1772748-18 .8584845-19
.1683522-17 $.1032836-17$ $.4875109-18$

$.1212234-16$ . 1211831-16 .1211018-16 .1209804-16 1198345-16 -1198345-16 -1167123-16 -1116479-16 1047381-16 -9605414-17 $.8575225-17$ $.7381502-17$ . 5988554-17 .4524165-17 .3256913-17 .2334306-17 -1665773-17 $.1159378-17$ $.7835343-1 A$ $.4872755-1 R$ 1613177-17 1612393-17 .1610935-17 .1609638-17 $.1594584-17$ .1552948-17 .1485920-17 .1395009-17 -1281623-17 .1148890-17 $.1003197-17$ -8500732-1A -7085421-18 -5811742-18 .4637994-1A -3616403-1A -2702633-1A -1909925-1A $.6024071-19$
-1520374-17 9332967-18 . 4412514-18 $.8141006-17$ - B138223-17 . $8132738-17$ 0132438-17 $.8124006-17$ $.7847754-17$ $.7837595-17$ $.7497635-17$ . $7033013-17$ .6448714-17 . $4946016-17$ . 4946016-17 -4039860-17 - 3194231-17 .2445907-17 - 1799932-17 -1311724-17 - 9269056-18 .6305037-18 -3968928-18 . $1891411-18$

$.9702839-18$ - $9697918-18$ . 9694954-18 $.9685137-18$ . 9592488-18 $.9342771-18$ $.8942561-18$ $0395413-18$ .7716997-18 $.6935834-18$ -693534-18 - $6070648-18$ $.5164021-18$ $.4318410-18$ - $2885791-18$ - 2891591-18 - $2246880-18$ - 1699957-18 -1217535-18 $.7888532-19$
$.3886715-19$ 
4488739-18

6553-18

4332292-18

4145158-16

-3892744-18

. $3584034-18$

3220258-18

2823815-15

2411692-18

$2029402-18$

$1676656-18$

$1676656-18$

$1361704-18$

$1080491-18$

$8084084-19$

$5786321-19$

$3813085-19$

$.1890341-19$ 


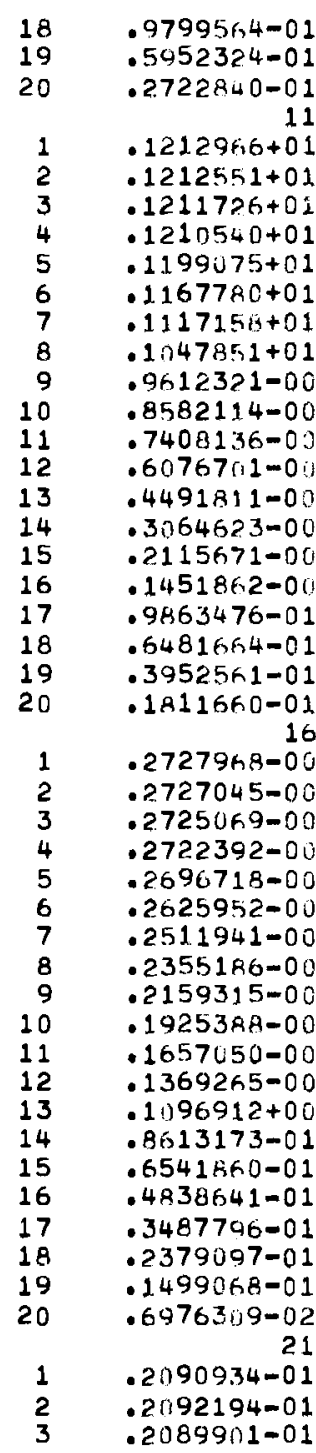

- $8833344-01$

$.5367948=01$

$.8340429-00$

- 8337615-00

. $8331990=00$

$.8323633-00$

-

- A244689-00

- $7679814=00$

- 7679814-00

- 7202407-00

.6602146-00

- $5888456-00$

$.5064451=00$

- $116000-00$

.3019339-00

.2069742-00

- $1452444=00$

$.1011970+00$

-6959432-01

. 4626411-01

.2840575-01

-1310024-01

$.1215320+00$

. $1215044+00$

- $1214234+00$

- $1212717+00$

- $1201413+00$

- $1170068+00$

. $1119200+00$

1050016to

- $0637629-01$

- $6624610=01$

- $74024610-01$

- 7492647-01

- $6303309-01$

- $191296-01$

- $4226020-01$

. $5556471-01$

-2556471-01

1881912-01

-1325594-01

. $3967471-02$
-8152003-01

4954623-01

$.6063061-00$

$.6061038-0$ n

.6056958-0n

.6050899-0n

.5993401-0n

5836763-0

5582545-0

$5235330-01$

$4798385-01$

4278482-0n

3676110-0

- $2970919-0$ n

2231622-0n

-1600196-0n

8111586-01

-5628046-01

3783481-01

$.2331848-01$

$.1082340-01$

7810529-01

$.7807055-01$

.7800178-01

$.7793804-01$

7720508-01

.7518723-01

.7193503-01

6751909-01

.6751909-01

5557949-01

$4850942-01$

$.4850942-01$

-4107503-01

3804516-01

- 2804516-01

1740096-09

1740096-01

-1298480-01

-9148456-02

-5896537-02

.2776777-0?
7359881-01 $.4476161-01$

$.4039465-00$ $.4038084=00$ $.4035375-00$ $.4031049-00$ $.3993074-00$ 3888540-00 . $37885251=00$ 3487805-00 -3196312-00 . $2850500-00$ - $2850500-00$ - $2445635-00$ - $1562366-00$ - $1568969-00$ - $1197556+00$ - B788827-01 .6377189-01 .4492505-01 -3042857-01 -1897113-01 $.8774492-02$

.4653472-01 $.4651116-01$ $.4649556-01$ $.4644680-01$ $.4600373-01$ $.4480461=01$ $.4287969-01$ .4025179-01 .3699276-01 $.3323727-01$ - $323727-01$ - $2907781=01$ 2472257-01 1713402-01 1380795-01 -1072706-01 - $1072706=01$ $.8099008-02$ -5776055-02 $.3711781-02$ 


$\begin{array}{rr}4 & .2087438-01 \\ 5 & .2167770-01 \\ 6 & .2014491-01 \\ 7 & .1927412-01 \\ 8 & .1809747-01 \\ 9 & .1665846-01 \\ 10 & .1496329-01 \\ 11 & .1311777-01 \\ 12 & .1119819-01 \\ 13 & .9419254-02 \\ 14 & .7777269-02 \\ 15 & .6305405-02 \\ 16 & .4994423-02 \\ 17 & .3735344-02 \\ 18 & .2667245-02 \\ 19 & : 1741740-02 \\ 20 & .8352372-03\end{array}$


ZONE

\begin{abstract}
AV FLUX
\end{abstract}
$.9980257-0 n$

$.5448032-00$

$.5995134-01$
AV POHER

$.1230547-15$

.6716426-16

$.2308208-17$
ACT 1

$.1904171+01$

$.1038406+01$

$.1136144+00$ 
BETA EFFECTIVE - - - - - - - 4.20647-03

NEUTRON GENERATION TIME - - - - - 3.02887-07 
(DELTA K) KK PER KG

DEL. $K$

$8937=02$

$.5455-02$

$5454-02$

$5446-02$
$5435-02$

5335-02

$5071-02$

4667-02

$4152-02$

3560-02

$.2930-02$

$.2301-02$

$.1714-02$

$6701-03$

$3264-03$

$1557-03$

$.7281-04$

$3304-04$

$1410-04$

$.5307-05$

$.1440-05$
DEL K

(SIGA)

DEL K

(SIGTR)

$.2461-07$

$.7413-07$
$.2113-06$

$.2113-06$
$.7864-06$

$.2424-05$

$.5893-05$

- $1216-\pi 4$

-1998-04

$.2866-04$

. 3741-04

$.4538-04$

5.312-04

$.7166-04$

$2894=04$

$1881-04$

$8306-05$

$3926-05$

$01000-05$

$.971 ?-06$

$.5630-06$

$.4501-06$
DEL K
(SIGJXK)

$. .1278-04$

-.1279-04

-.1277-04

-.1275-04

-.1253-04

-.1113-04

$-.1009-04$

-.8949-05

-.7795-05

$-.6744-05$
$-.5844-05$

$-.5188-05$

-.4143-05

$-.2610-05$

-.1445-05

$-.7430-06$

-.3566-06

-.1572-06

$-.5904-07$
$-.1316-07$
DEL $K$

5455-02

5454-02

5450-02

5443-02

5350-02

5176-02

4932-02

4629-02

4280-02

3901-02

3507-02

3114-02

2736-02

2404-02

.2096-02

1828-02

1601-02

$.1410-02$

$.1250-02$

.1115-02

$.1001-02$
AVG RADII

AVG AXII

.3275-00

-9525-00

$.1587+01$

$.4716+01$

$.9068+01$

$.1342+02$

$-1777+02$

$.2212+02$

$.2648+02$

$.3083+02$

$.3518+02$

$.3953+02$

$.4361+02$

$.4742+02$

$.5123+02$

$5504+02$

$.5885+02$

$6266+02$

$6647+02$

$.7028+02$
.3175-00 $.3175-00$ . $3175-00$ -3175-00 $.3175=00$ -3175-00 .3175-00 .3175-00 .3175-00

.3175-00

3175-00

3175-00

3175-00

.3175-00

$.3175=00$

$.3175-00$

$.3175-00$

.3175-00

.3175-00

3175-00

.3175-00 
(OELTA K)/K PER KG

UEL K
(1.USIGF)
$.8735-02$
$.8728-02$
$.8717-02$
$.8699-02$
$.8538-02$
$.8103-02$
$.7424-02$
$.6545-02$
$.5527-02$
$.4435-02$
$.3342-02$
$.2297-02$
$.1302-02$
$.6314-03$
$.3093-03$
$.1482-03$
$.6894-04$
$.2999-04$
$.1124-04$
$.2443-05$

DEL $K$
(SIGA)

$-.3389-02$ $-.3387-02$ $-.3382-02$ $-.3376-02$ $-.3312-02$ $-.3142-02$ -.2875-02 $-.2530-02$ $-.2127-02$ -.1693-02 -.1255-02 $-.1255-02$
$-.8325-03$ $-.8325-03$ $-.4060-03$ $-.1968-03$
$-.9115-04$ $-.4183-04$ $-.1889-04$ $-.8056-05$ $-.2990-05$
$-.6573-06$

\section{DEL K
(SIGJXK}

1253-04

-.1252-04

-1251-04

$-.1249-04$

$-1229-04$

$-.1175-04$
$-.1093-04$

-.1093-04

$-.9924-05$
$-.8838-05$

$-.8838-05$
$-.7817-05$

$-7817-05$
$-.6953-05$

$-.6953-05$
$-.6400-05$

$-.6400-05$
$-.5366-05$

$-.5366-05$
$-.3575-05$

-.3575-05

-.2081-05

- 1119-05

$-.5605-06$
$-.2575-06$

-.2575-06

$-.1007-06$
$-.2331-07$
DEL K
(INTG)

5335-02

. 5333-02

. 5330-02

. 5326-02

$.5257-02$

$.5140-02$

$.4976-02$

$.4768-02$

$.4527-02$

$4260-02$

3979-02

3695-02

3440-02

-3440-02

$.3192-02$

$.2964-02$

2760-02

- $2580-02$

- 2421-02

$.2279-02$
$.2153-02$
AVG RADII

$.4716+01$

$.4716+01$

$.4716+01$

$.4716+01$

$.4716+01$

$4716+01$

$4716+01$

$.4716+01$

$4716+01$

$.4716+01$

$4716+01$
$4716+01$

$.4716+01$

$.4716+01$

$.4716+01$

$.4716+01$

$.4716+01$

$.4716+01$

$4716+01$

$.4716+01$
$.4716+01$
AVG AXII

-3175-00 $9525-00$
$1587+01$

$.2222+01$

$.4767+01$

$.9222+01$

$.1368+02$

- $1813+02$

$.2258+02$

$2704+02$

$.3149+02$

$.3595+02$

$455+02$

$4008+02$

$.4389+02$

-5151+02

$.5151+02$

$.5532+02$
$.5913+02$

-5913+02

$.6294+02$
$.6675+02$ 
(DELTA K)/K PER KG

DEL K
(NUSIGF)

DEL $K$

(SIGA)

.0000

.0000

.0000

- 0000

.0000

.0000

.0000

.0000

.0000

.0000

.0000

.0000

.0000

.0000

.0000

.0000

.0000

.0000
.0000
$-.2791-04$

$-.2789-04$
$-.2785-04$

$-.2785-14$
$-.2779-04$

-.2726-04

$-.2587-04$
$-.2373-04$

$=.2098-04$

$-.2098-04$
$-.1779-04$

$-.1739-04$

$-.1096-04$

$-.7729-05$

$-.4804-05$
$-.2291-05$

$-.2291-05$

$-.3626-06$

$-.1490-06$

$-.6145-07$

$-.2416-07$

$-.8338-08$

-.1697-08
DEL K

(SIGTR)

-1112-06

$.3334-06$

$.3601-05$

.1111-04

$.2699-04$

$.9142-04$

$.1310-03$

$-1310-03$

-1707-03

-2066-03

- $3241=03$

- 324a-03

-1311-03

$.8611-04$

-1836-04

.8948-05

$.4595-05$

$.2673-05$
$.2140-05$
DEL K
(SIGUXK)

$. .1495-03$

-.1496-03

$.1495-03$
$-.1492-03$

$.1467-03$

$-1467-03$
$-.1400-03$

$-.1302-03$

$-.1181-03$

-.1181-03

$-.11047-03$
$-.9122-04$

$-.9122-04$
$-.7892-04$
$-.6839-04$

$-.6839-04$

-.6071-04

-.4A49-04

-.3055-04

-.1691-04

-.8695-05

-.4174-05

-.1840-05

-.6909-06

$. .1540-06$
DEL K
(INTG)

1773-03

$-.1772-03$

$-1767-03$
$-1753-03$

-.1645-03

$-1645-03$
$-1485-03$

-.1244-03

$-.9457-04$

$-.9457-04$
$-.6097-04$

$-.6997-04$
$-.2558-04$

$.9486-05$

$.4361-04$

-8628-04

.8532-04

-8077-04

.7254-04

$.6439-04$

$.5714-04$

. 5091- 04

$.4561-04$
$.4112-04$
AVG RADI I

.3175-00

.9525-00

$.1587+01$

$.2222+01$

$.9068+01$

$.1342+02$

$1777+02$

$.1777+02$

$.2648+02$

- $3083+02$

$.3083+02$

$.3518+02$

- $453+02$

$.4361+02$

$.4742+02$

$.5123+02$

$.5504+02$

$.5885+02$

$.6266+02$

$.6647+02$

$.7028+02$
AVG AXII

.3175-00

$.3175-00$

$.3175-00$
$.3175=00$

$.3175=00$

$.3175=00$

.3175-00

.3175-00

. $3175-00$

.3175-00

$.3175-00$

-3175-00

- $3175-00$

- 3175-00

-3175-00

- $3175-00$

. $3175-00$

$.3175=00$

-3175-00

$.3175-00$
$.3175-00$ 
BNWL- 409

APPENDIX C

FORTRAN-IV SOURCE DECK LISTING 


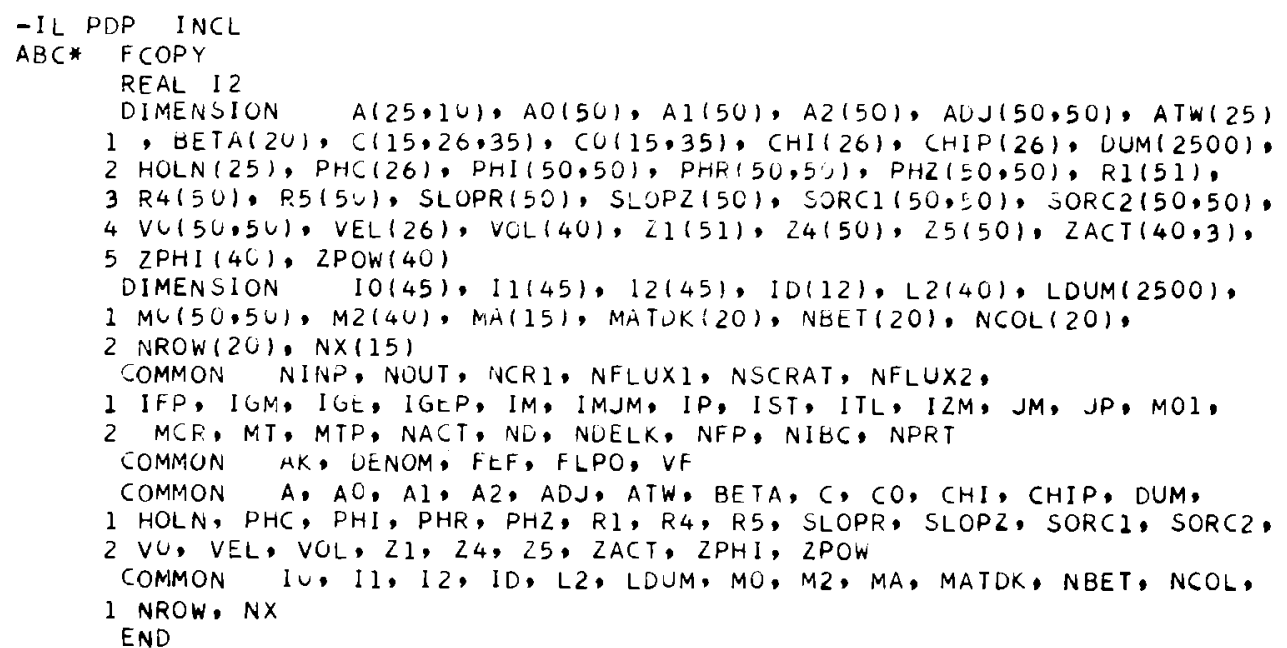




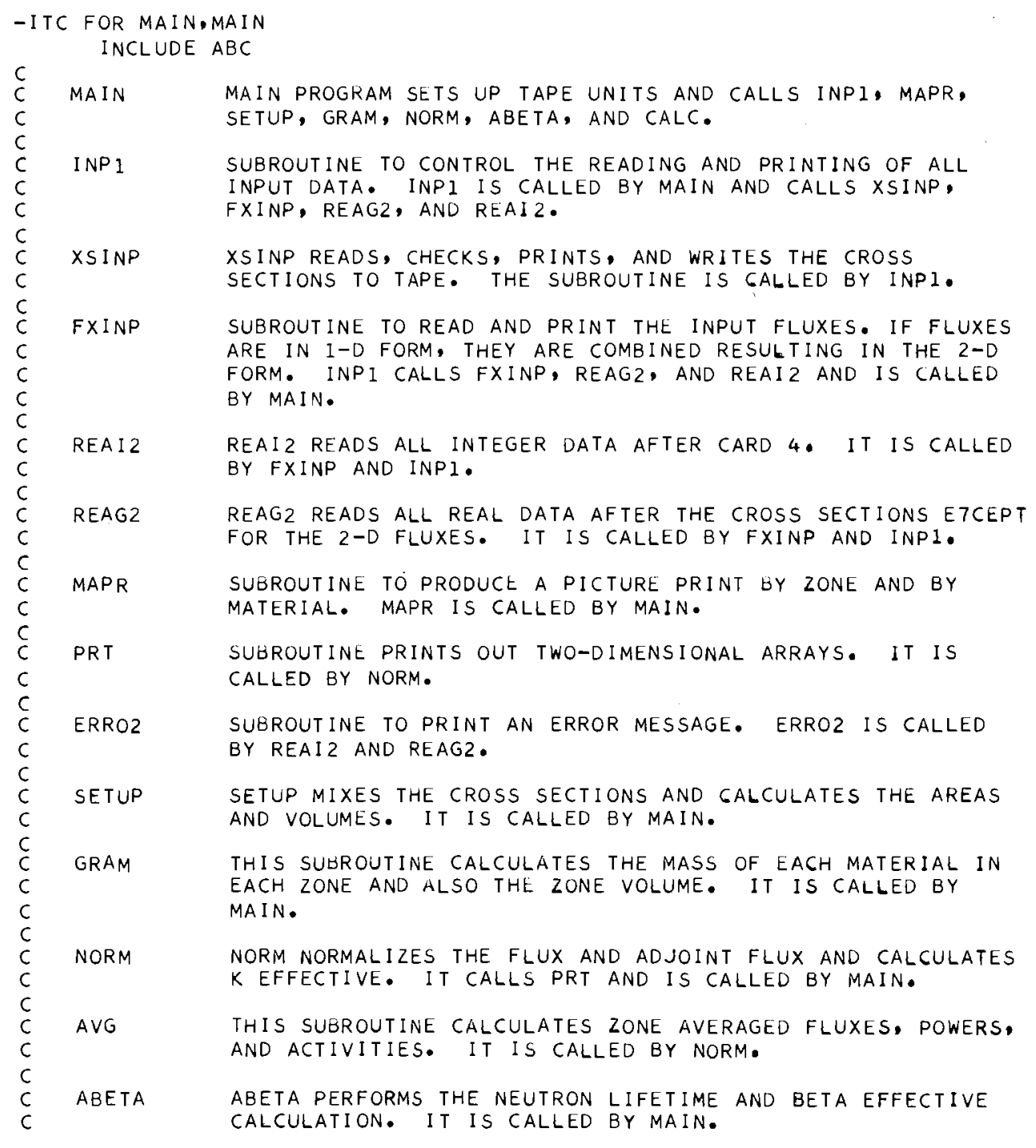


CALC SUBROUTINE TO CALCULATE AND PRINT REACTIVITY COEFFICIENTS. IT IS CALLED BY MAIN.

AK CALCULATED VALUE OF K EFFECTIVE

CAD TOTAL CHI \#ADJOINT AT CENTER

CFX TOTAL CENTER POINT FLUX

CPW TOTAL CENTER POINT POWER

DENOM DENOMINATOR OF PERTURBATION FORMULA

FEF AVERAGE ENERGY RELEASED PER FISSION (MEVIFISSION)

FLPO FLUX OR POWER (SEE NFP)

IFP FLUX PUNCH OPTION

I GE

I GEP

$1 G M$

NUMBER OF GROUPS (L/E 26

NUMBER OF GROUPS PLUS 1

NUMBER OF RADIAL INTERVALS IL/E 50 )

$I M * J M$

IM +1
NUMBER OF DOWNSCATTERING TERMS

NUMBER OF DOWNSCATTERING TERMS IL/E 101

CROSS SECTION TABLE LENGTH (L/E 15

NUMBER OF MATERIAL ZONES (L/E 40

NUMBER OF AXIAL INTERVALS (L/E 50$)$

LENGTH OF MIX VECTORS (IO,I1, I2) (L/E 45)

VUMBER OF MATERIALS FROM CARDS (L/E 25 )

TOTAL NUMBER OF MATERIALS INCLUDING MIXES (L/E 35)

NUMBER OF MATERIALS FROM TAPE

NUMBER OF ACTIVITY TRAVERSES (L/E 3)

CROSS SECTION TAPE

FLUX INPUT OPTION

NUMBER OF REACTIVITY COEFFICIENT CALCULATIONS IL/E 20 ,

FLUX TAPE

ADJOINT FLUX TAPE

FLUX NORMALIZATION OPTION

NUMBER OF ISOTOPES FOR BETA CALCULATION (L/E 20)

LOG ICAL INPUT TAPE

LOGICAL OUTPUT TAPE

PRINT OPTION

SCRATCH TAPE

TOTAL REACTOR POWER

ABSORPTION RATE

RADIAL LEAKAGE

RLEAK $\quad$ LEAKAGE RATE

RSORS 


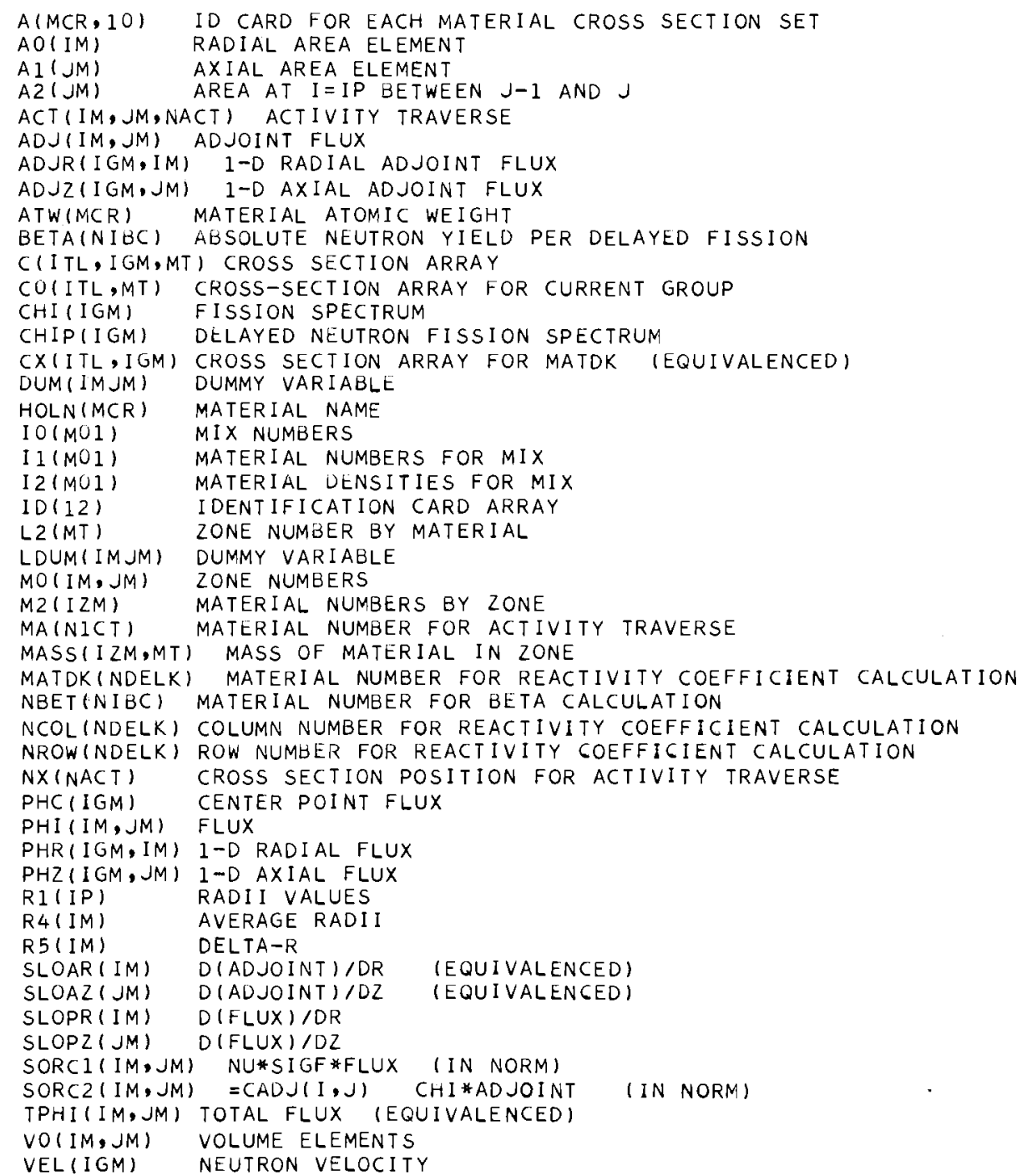




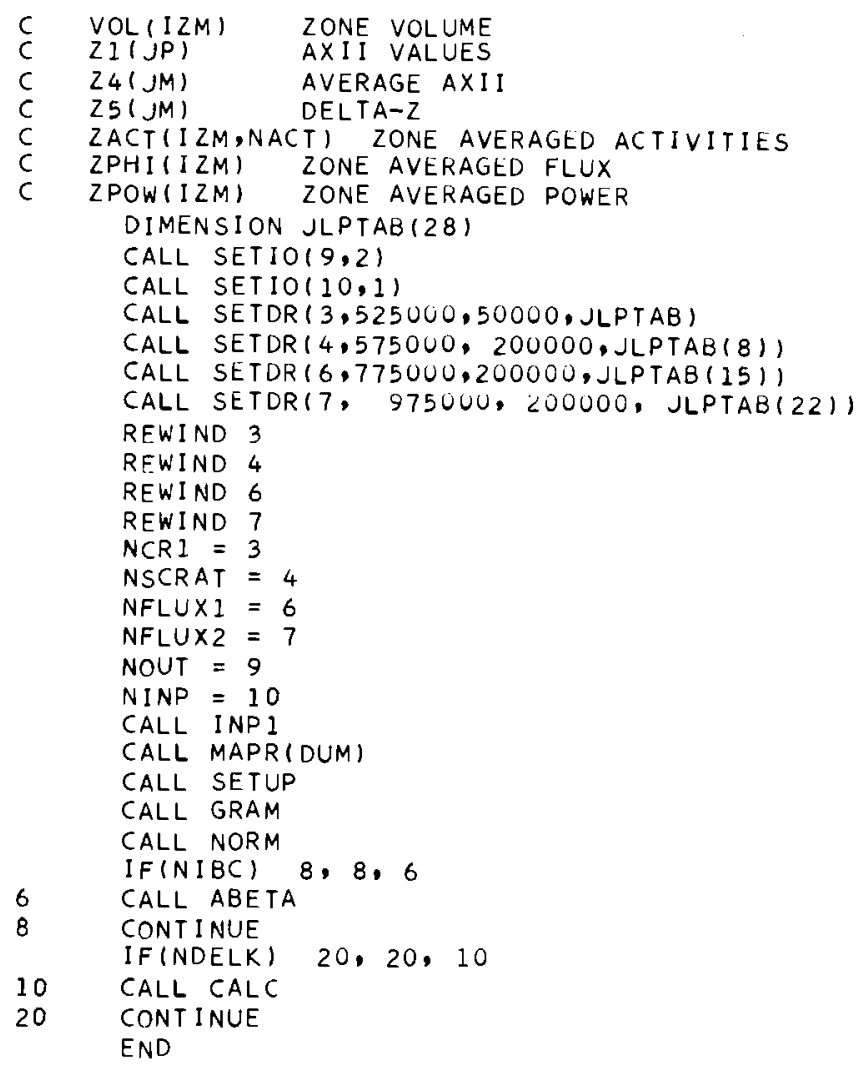


-ITC FOR INPI, INPI

SUBROUT INE INP

INCLUDE ABC

C THIS SUBROUTINE CONTROLS THE READING OF ALL INPUT DATA

WRITE (NOUT, 3$)$

- I V * * * * 1/1111

READ (NINP, 5$)$ (IDI), I $=1,12$ )

FORMAT (12A6)

WRITE(NOUT, 5) (IDII), I = 1,12

WRITE(NOUT, 6)

FORMAT (IH

READ (NINP, IO) ND, IGM, IST, IFP, IM, JM, MCR, MTP, IZM, MT,

I MOI, NPRT, NDELK, NIBC, NACT,NFP, IGE

10. FORMAT (1216)

READ (NINP,15) FLPO,VF,FEF

FORMAT $(6 \mathrm{E} 12.6)$

WRITE NOUT, 201 ND, IGM, IST, IFP, IM, JM

FORMAT (78H ND $1 / 2 / 3 / 4 / 5 / 6=$ FLUXES FROM TWO $1-D$ RUNS/FLUXES

I FROM 2-D RUN/FLUX FROM/, 12X,66H1-D,ADJOINT FROM 2-D/FLUX FROM 2-D 2 , ADJOINT FROM I-D/FLUX FROM I-D, /12X, 35HNO ADJOINT/FLUX FROM 2-D, N 30 ADJOINT, 34X,19/

NUMBER OF ENERGY GROUPS, $46 \mathrm{X}, \mathrm{I} 9 /, 42 \mathrm{H}$

5IST NUMBER OF DOWNSCATTERING TERMS, 39X, I9/,

$661 \mathrm{H}$ IFP O/1/2=NO EFFECT/PUNCH FLUXES/PUNCH ADJOINT FLUXES,

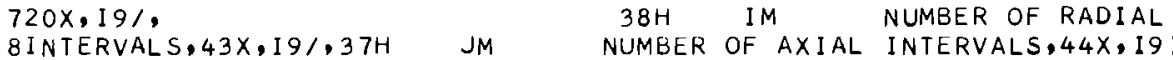
8INTERVALS, $43 x, 19 /, 37 \mathrm{H}$ JM NUMBER OF AXIAL INTERVALS, 44X,I9)
WRITEINOUT, 30 ) MCR, MTP, IZM, MT, MOI, NPRT

30 FORMAT $142 \mathrm{H}$ MCR NUMBER OF MATERIALS FROM CARDS, 39X,19/,41H 1 MTP NUMBER OF MATERIALS FROM TAPE, 40X,19\%36H IZM NU 2MBER OF MATERIAL ZONES, $45 x, 19 /, 53 \mathrm{H}$ MT TOTAL NUMBER OF MATE 3RIALS INCLUDING MIXES, $28 \mathrm{X}, 19 / .44 \mathrm{H}$ MOI NUMBER OF MIXTURE SPE 4CIFICATIONS,37X,I9/,54H NPRT PRINT OPTION10/1=PARTIAL PRINT/ 5FULL PRINTI27X,I9/1

WRITE (NOUT, 40) NDELK, NIBC, NACT

40 FORMAT $166 \mathrm{H}$ NDELK $272 \mathrm{H}$ NIBC $15 X, \mathrm{O}=$ NO EFFECT/USE N MATERIALS FOR BETA EFFECTIVE CA 3LCULATION, $9 X, 191$

$449 \mathrm{H}$ NACT U/N=NO EFFECT/DO N ACTIVITY TRAVERSES, 32X,I91

WRI TE (NOUT, 50 ) NFP

FORMAT (78H NFP FLUX OR POWER NORMALIZATION $11 / 2 / 3 / 4=C E N T$ IER PT. FLUX/CENTER PT. $1,12 X, 46$ HPOWER/TOTAL POWER/DENOMINATOR OF $P$ 2ERT FORMULA), 23X,I9,

WR ITE (NOUT, 551 I IGE

WR I TE (NOUT, 55) IGE

FORMAT 46H IGE GEOMETRY $(0 / 1 / 2=R-Z / X-Y / R-T H E T A), 35 X, I 9 /$ WRITE (NOUT, 60) FLPO,VF, FEF

FORMAT 1 $41 \mathrm{H}$ FLPO FLUX(N/CM2-SEC) OR POWER(MWT),39X,1PE10.5/4

$17 \mathrm{H} V \mathrm{VF}$ VOLUME FACTOR (VOLUME = VF*PI*R2*Z) $133 \times, 1 \mathrm{PE} 10.5$ ) 
64

244H FEF ENERGY(MEV) RELEASED PER FISSION, 36X,1PE 10.51

FORMAT (IHI, 12A6///)

C READ, CHECK, PRINT, AND WRITE CROSS SECTIONS TO TAPE

WRITE (NOUT, 64) (ID(I), I = 1,12

C READ FLUXES

WRI TE (NOUT, 64) (IDII), I $=1,121$

WRITE (NOUT, 82 )

82

作

IAL FLUX/RADIAL ADJOINT FLUX/AXIAL ADJOINT

$232 \mathrm{H}$ FLUX/2-D FLUX/2-D ADJOINT FLUX) i)

CALL FXINP

$I P=I M+1$

C READ RADIIR

84 WRITE (NOUT, 84)

BOUNDARIES (RI/ZI=RADIAL POINTS/AXIAL POINTS) CALL REAG2 $(6 H$ RI,RI,IP)

C READ AXI I

CALL REAG216H

$21,21, J P 1$

$C$ READ ZONE NUMBERS

WR I TE (NOUT • 86)

86 FORMAT(3OHUZONE NUMBERS BY MESH INTERVAL) CALL REAI2 (6H MO,LDUM, IMJM)

$L=0$

DO $87 \quad J=1, J M$

DO $87 \quad I=1, I M$

$\mathrm{L}=\mathrm{L}+1$

87 MOLII,J) $=$ LDUM(L)

WRITE (NOUT, 88)

FORMAT (25HOMATERIAL NUMBERS BY ZONE)

CALL REAI2 (6H M2,M2,IZM)

C READFISSION FRACTIONS

WRITEINOUT O

WRITEINOUT, 90)

ISSION SPECTRUMI

CALL REAG2 (6H CHI, CHI,IGM)

6 READ MIXTURE SPECIFICATIONS

92 WORMAT(82HOMIXTURE SPECIFICATIONS 1I0/I1/I2=MIX NUMBER/MAT. NUMBER 1 FOR MIX/MATERIAL DENSITY)

CALL REAI2 (6H IO,IO,MO1)

CALL REAI2 $16 \mathrm{H}$ I1,II,MO1)

$C$ CHECK FOR LIFETIME AND BETA CALCULATIONS

IF (NIBC) 1 120,120, I00

$C$ READ MATERIAL NUMBERS FOR BETAS 
100 WRITE (NOUT, 94)

作

CALL REAI216H NBET, NBET, NIBC

C READ BETAS

CALL REAG2 $16 \mathrm{H}$ BETA,BETA,NIBC

C READ DELAYED FISSION SPECTRUM

WRITE (NOUT, 102 )

102 FORMAT(25HODELAYED FISSION SPECTRUM)

CALL REAG2(6H CHIP, CHIP,IGM)

C READ VELOCITIES

104 FORMAT(ITHONEUTRON VELOCITY)

CALL REAGZI (GH VEL, VEL, IGM)

C CHECK FOR DELTA K CALCULATION

120 IF (NDELK) $160,160,140$

C READ MATERIAL NUMBERS, ROW NUMBERS, AND COLUMN NUMBERS FOR

C PERTURBATION CALCULATION

140 WRITE (NOUT, 138 )

138 FORMAT (98HOPERTURBATION CALCULATION INFORMATION (MATDK/NROW/NCOL $=M$ IATER I AL NUMGER/ROW/COLUMN TO BE PERTURBED))

CALL REAI2 $(6 \mathrm{H}$ MATDK. MATDK ANDELK)

CALL REAI2 $(6 \mathrm{H}$ NROW, NROW, NDELK,

CALL REAI2(6H NCOL, NCOL, NDELK)

C CHECK FOR ACTIVITIES

160 IF (NACT) $1000,1000,180$ PEAT MATERIAL NUMGERS AND XS POSITION FOR ACTIVITIES

180 WRITE(NOUT, 178 )

INOACTIVITY TRAVERSE INFORMATION (MA/NX=MATERIAL NUMBER/CR IOSS SECTION POSITIONI)

CALL REAI216H

CALL REAI2(6H NX, NX, NACT)

1000 CONTINUE

RETURN

END 
-ITC FOR XSINP,XSINP SUBROUTINE XSINP

INCLUDE ABC

C THIS SUBROUTINE READS, CHECKS, PRINTS, AND WRITES THE CROSS SECTIONS TO TAPE IF (MCR) $500,500,10$

C READ CROSS SECTIONS FROM CARDS

10 DO $50 \quad \mathrm{I}=1, M C R$

READ(NINP, 20$)$ HOLN(I), ATW(I), (A(I,J), J=1, 10$)$

FORMAT $(A 6, E 6.2,10 A 6)$

DO 40 IIG=1, IGM

READ (NINP, 3U) (C(L,IIG,I), L= I, ITL)

FORMAT $(6 E 12 \cdot 5)$

50 CONTINUE

DO $200 \quad J=1, M C R$

DO $200 \quad 1=1,1 \mathrm{GM}$

$G=C(2, I, J)+C(5, I, J)$

DO $80 \quad K=1$, IST

$K K=1+K$

$M=5+K=70,70,80$

$G=G+C(M, K K, J)$

CONT I NUE

IFUABS(IG - $C(4, I, J)) / C(4, I, J))-.01) 200,90,90$

WRI TE (NOUT, IIU) J,I

IIU FORMATIIH/,16H CHECK MATERIAL I $2,5 X, 7 H$ GROUP I 2$)$

200 CONTINUE

C PRINT CROSS SECTIONS

IF(NPRT) $210,210,250$

WRITE (NOUT, 220)

220 FORMAT(38H THE FOLLOWING NUCLIDES ARE FROM CARDS $/ 1$

240 DO $240 \quad K=1, M C R \quad K, \operatorname{HOLN}(K),(A(K, J), J=1,10)$

245

FORMAT (I9,6X,11A6)

GO TO 510

250 D6 $480 \quad K=1$, MCR

FORMAT 1 HI

245) K, HOLN(K), $(A(K, J), J=1,10$

WRITE (NOUT, 260 )

260

$\begin{array}{lcccc}2113 H \text { GROUP } & \text { SIGF } & \text { SIGA } & \text { NUSIGF } & \text { SIGTR } \\ 36 & \text { G-2XG } & \text { G-3 } & \text { SIGXG }\end{array}$

$6-1 \times$

IF(IST - 6) 290, 290, 265

DO $270 \quad I=1, I G M$

268 FORMATI 3 , IIF 10.3$)$

270 WRITE(NOUT, 268$)[,(C(J, I, K), J=1,11)$

WRITE (NOUT,275)

275 FORMAT (44HIGROUP G-7XG G-8XG G-9XG G-10XG/, 


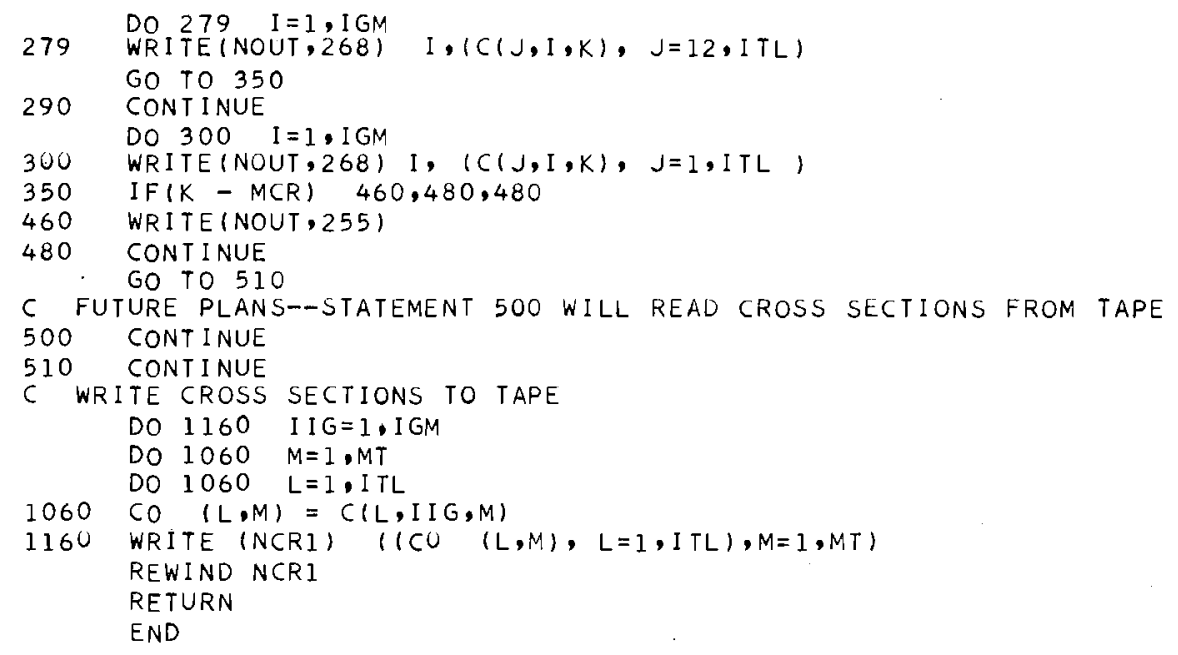


-ITC FOR FXINP, FXINP

SUBROUTINE FXINP

INCLUDE ABC

INTEGER TEMP

C THIS SUBROUT INE RLADS FLUXESIREGULAR FLUX AND ADJOINT FLUXI IF THEM IN $2-D$ FORM
TMUM

$I M J M=I M * J M$

$J J R=I G M * I M$

$J U=I G M * J M$

$\angle L=$

NND $=N D$

$N D=I A B S(N D)$

C LLH $=1 / 2=$ REGULAR FLUX/ADJOINT FLUX

GO TO $(100,860,100,800,100,800)$, ND

C FLUX IS IN I-D FORM

DO CONT INUE

$Z F X=0.0$

$R F X=0.0$

$120)$, LLH

110 CALL REAG2 $(6 \mathrm{H}$ PHR,DUM,JJR)

GO TO 130

120 CALL REAG2 6 H ADJR,DUM, JJR

IF NND IS POSITIVE, AUJOINT FLUXES ARE REVERSED BY GROUP IF (NND) 130, 130, 12

121 CONTINUE

$\mathrm{L}=0$

DO $126 K=1$, IGM

DO $124 \quad I=1, I M$

$\mathrm{L}=\mathrm{L}+\mathrm{I}$

$K K=I G M+1-K$

$124 \operatorname{PHR}(K K, I)=\operatorname{DUM}(L)$

126 RFX $=$ RFX + PHR $(K K, 1)$

GO TO 165

$130 \mathrm{~L}=0$

DO $160 \mathrm{~K}=1, \mathrm{IGM}$

DO 158 I $=1$, IM

$L=L+1$

RFX + PHR(K, l $)$

CONT I NUE

GO TO (I7O, 180), LLH

170 CALL REAGZ16H PHZ,DUM,JJZ)

GO TO 190

180 CALL REAGZ(6H ADJZ,DUM,JJZ)

IF (NND) 190, 190, 182

C SWITCH GROUPS

182 CONTINUE 
WRITE (NOUT, 818) ((PHI\{I, J), I = I, IM), J = I, JM)

WRI TE (NFLUXI) ( $(P H I(I, J), I=1, I M), J=1, J M)$

840 CONTINUE

GO TO $(910,850)$, LLH

850 CONTINUE

IF NND IS POSITIVE, ADJOINT FLUXES ARE REVERSED BY GROUP

IF(NND) $910,910,860$

860 CONT INUE

REWIND NFLUXI

DO $880 \quad K=1$

READ (NFLUX)

DO $900 K=1$, IGM

BACK SPACE NFLUXI

READ (NFLUXI) (PHI I, J), I = $1, I M), J=1, J M)$

WRITE (NSCRAT) ((PHI $(I, J), I=I, I M), J=1, J M)$

BACKSPACE NFLUXI

900

CONT I NUE

REWIND NSCRAT

REWIND NFLUX 1

TEMP $=$ NSCRAT

NSCRAT = NFLUXI

NFLUX1 = TEMP

910 CONTINUE

1000 GO TO (1100, 2200), LLH

C SWITCH TAPE DESIGNATIONS

TEMP $=$ NFLUX

NFLUX1 = NFLUX2

NFLUX2 = TEMP

C RE D ADJOINT FLUX

GO TO $(100,800,800,100,2000,2000)$, ND

C THERE IS NO ADJOINT FLUX INPUT

2000 CONTINUE

$A A A=1.0$

AAA $=1.0 K=1$, IGM

DO $2100 K=1, I G M A, I=1, I M), J=1, J M I$

2100 CONTINUE

2200 REWIND NFLUXI

REWIND NFLUXI

C SWITCH TAPE DESIGNATIONS

TEMP $=$ NFLUX2

NFLUX2 $=$ NFLUX]

NFLUX1 = TEMP

RETURN 


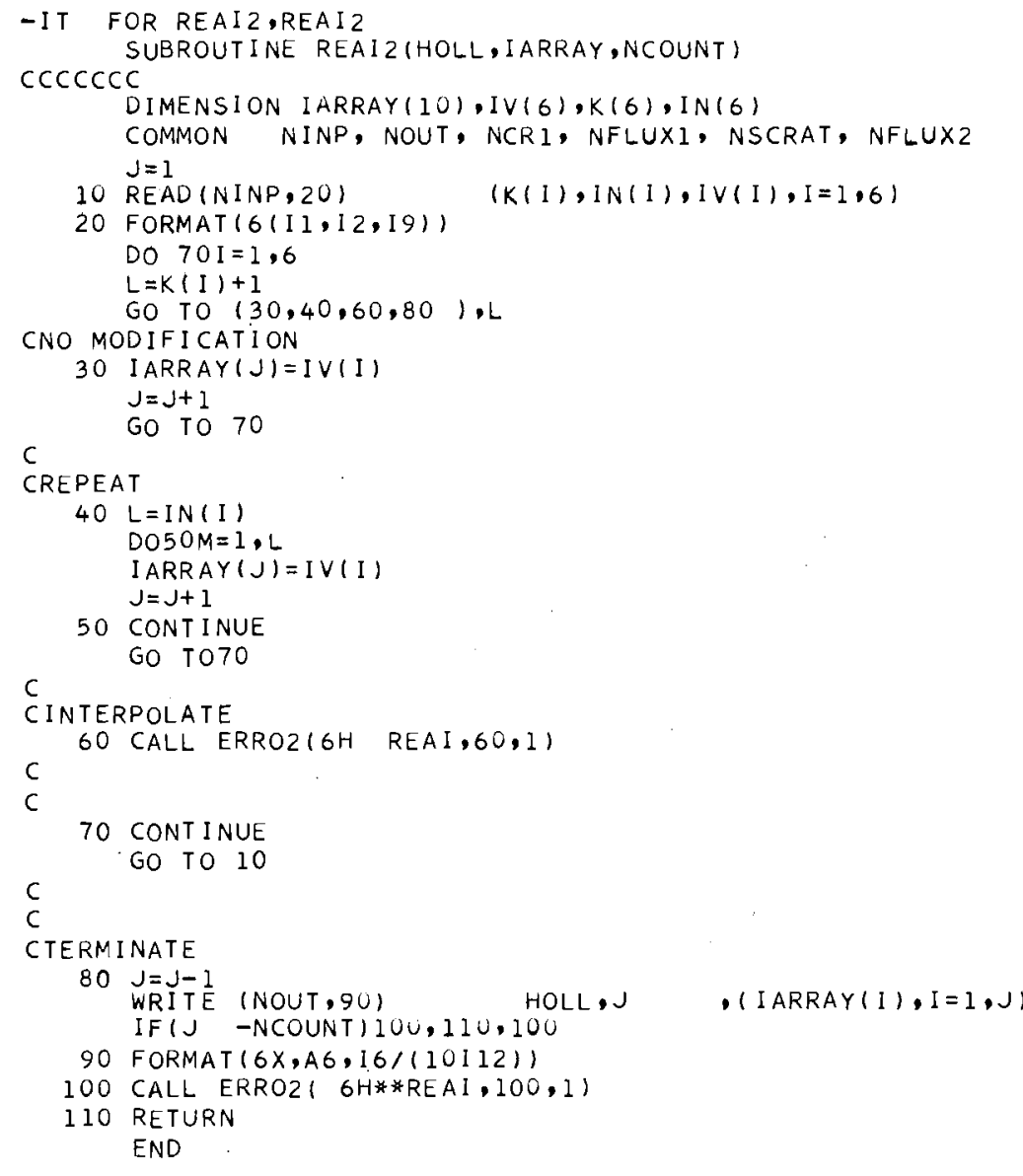




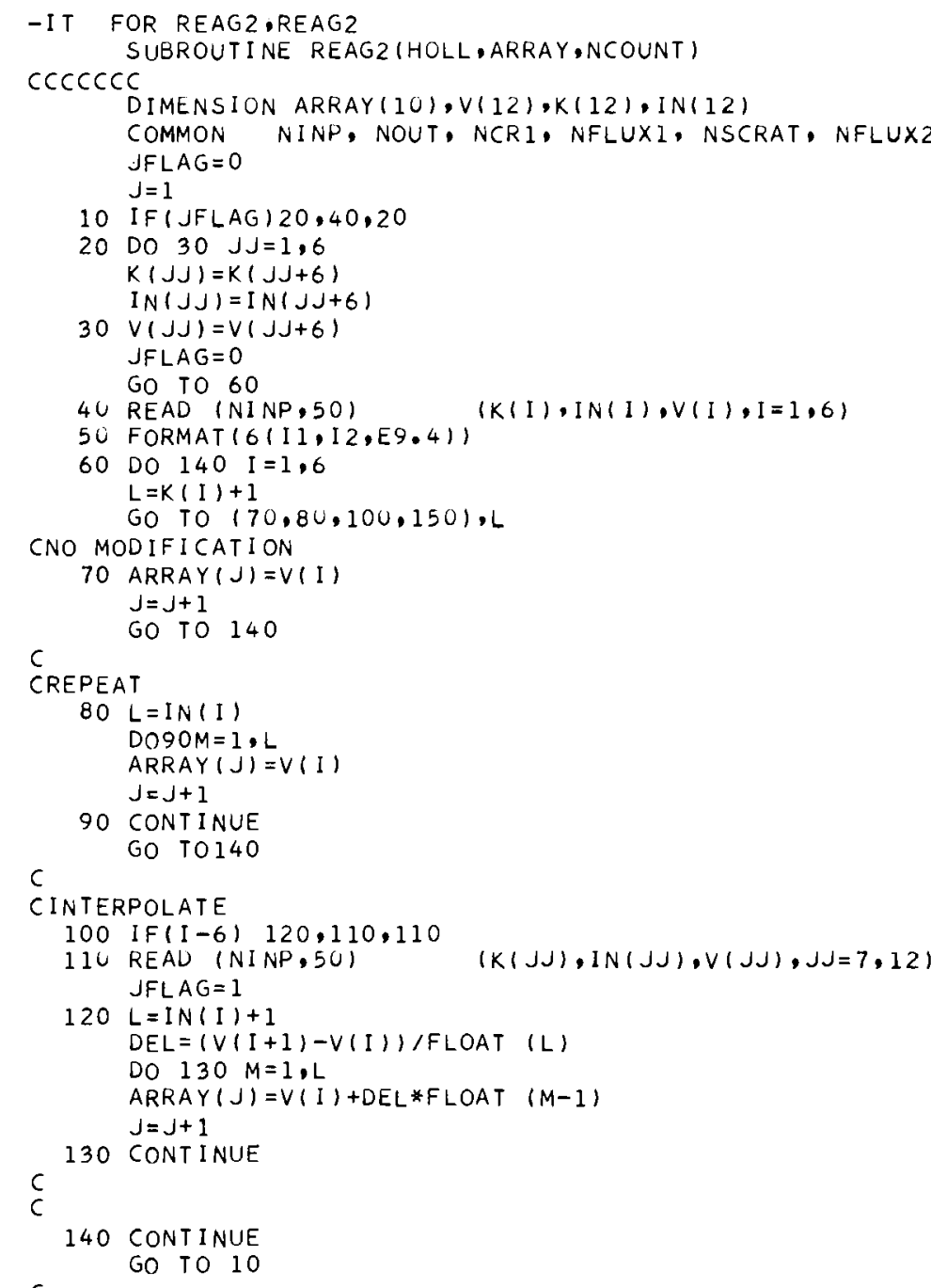


CTERMINATE,

$20 \quad j=J-1$

WRITE (NOUT, 160) HOLL, J

IFIJ -NCOUNTI17U,180,170

160 FORMAT $(6 X, A 6$, I $6 /(1 \cup E 12.5))$

170 CALL ERRO21 6H**REAG,170,1

180 RETURN

END 
-ITC FOR MAPR, MAPR

SUBROUTINE MAPR(K)

INCLUDE ABC

DIMENSION K(9)

$1 \quad I X=I M$

WRITE (NOUT,6) (IDII), I $=1,12$ )

6 FORMAT $(1 \mathrm{HI}, 12 \mathrm{AG} / / /)$

3 DO 5 J J J I I I I I

5 WRITE (NOUT, 4 )

4 FORMAT ( 5 H 4$)$

W5II2)

WRITE (NOUT, 15

$\mathrm{X} / 2 \mathrm{H} \mathrm{I} / 2 \mathrm{H} \mathrm{A} / 2 \mathrm{H} \mathrm{L} / / 8 \mathrm{H}$ RADIAL

(NOUT,6) (IU(I), I $=1,12)$

DO $10 \mathrm{JJ}=1, \mathrm{IY}$

$J=I Y-J J+1$

DO $11 L=1, I X$

$N=M O(L ; J)$

$1 \quad K(L)=I A B S \quad(M 2(N)$

WRITE (NOUT, 4 )

WRITE INOUT, 15

$(K(L), L=1, I X$

RETURN

END 
-IT FOR PRT,PRT

SUBROUTINE PRT(IM, JM, PHI)

COMMON NINP, NOUT, NCR1, NFLUX1, NSCRAT, NFLUX2

DIMENSION PHI (50, 50$)$

DO $60 \quad I=1$, IM,

I $1=I$

$12=I+4$

$I F(I 2-I M) \quad 20,20,10$

$10 \quad 12=I M$

$1012=1 M$

20 WRITE ( NOUT,30)

FORMAT $(5 X, 5$ I 20$)$

DO $50 \quad J J=1, J M$

WRITE (NOUT, $40, \mathrm{~J},\{$ PHI $(K, J), K=I 1, I 2)$

40 FORMAT $(5 X$, I $5, E 15.7,4$ E20.7)

50 CONT I NUE

RETURN

END 
-IT FOR ERRO2,ERRO2

SUBROUTINE ERROZ( HOL, JSUBR, I)

COMMON NINP, NOUT, NCRI, NFLUXI, NSCRAT, NFLUX2

WRITE (NOUT,1)

1 FORMAT $(2 \mathrm{H} * / 9 \mathrm{H}$ ERROR IN,A6,3H AT, I6/2H*/2H*)

GO TO $(3,4)$, I

3 STOP

4 RETURN

END 


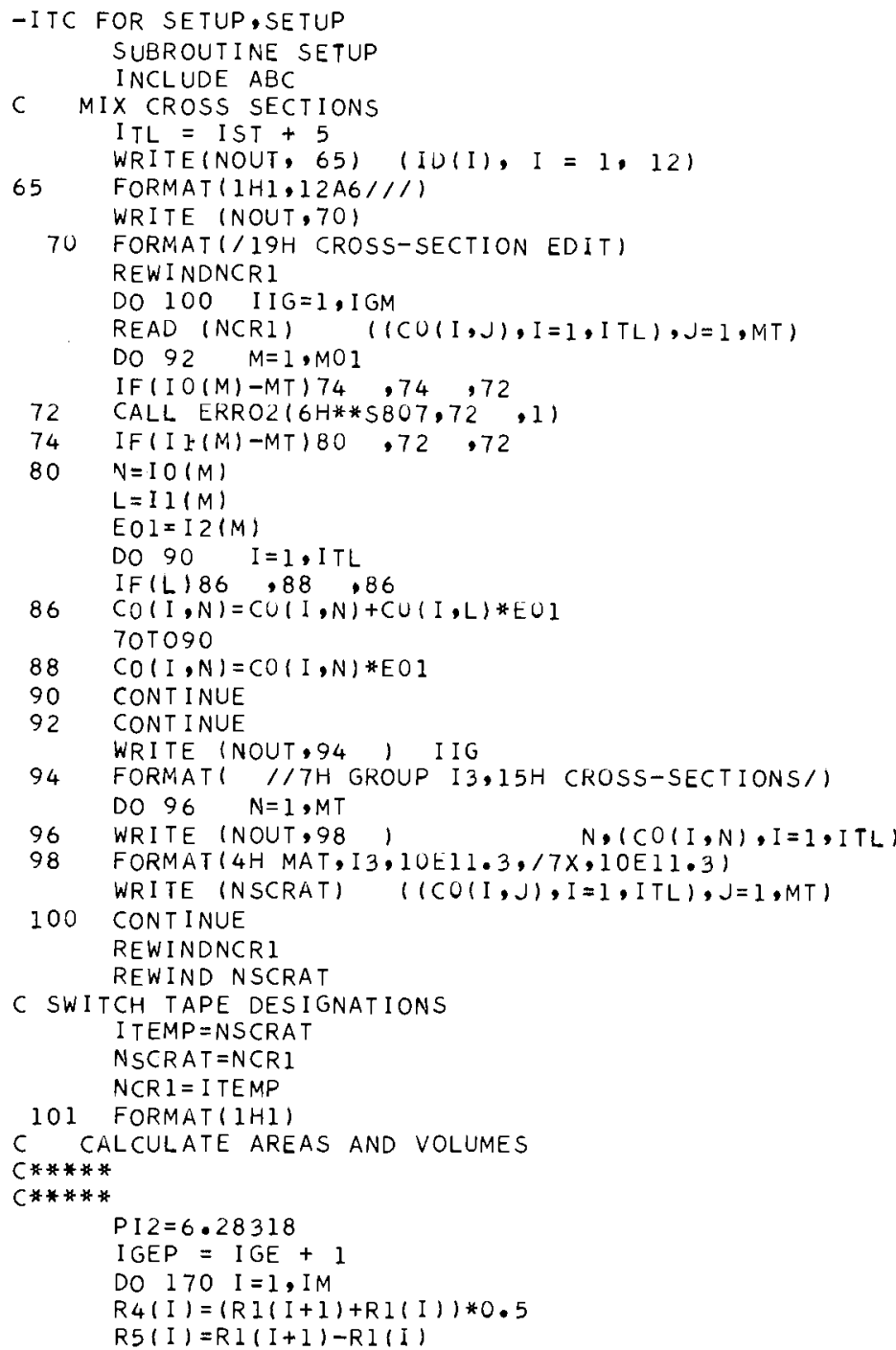


IF( R5(I) ) 130, 130, 140

CALL ERRO2 $(6 H * R 5(1), 130,1)$

CONTINUE

GO TO $(160,150,150)$, IGEP

150 CONTINUE

AI $(I)=R 5(1)$

GO TO 170

CONTI NUE

AI (I) =PI 2*R5(I)*R4(I)

CONTINUE

DO $200 \mathrm{~J}=1$, JM

$24(J)=(21(J+1)+21(J)) * 0.5$

$25(J)=21(J+1)-21(J)$

$1 F(25(J) 1175,175,180$

175 CALL ERRO2 $(6 H * 25(J), 175,1)$

180 CONTINUE

GO TO $(183,182,183)$, IGEP

$A 2(J)=25(J)$

GO TO 184

$A 2(J)=P I 2 * R 1(I P) * 25(J)$

184 DO $195 \mathrm{I}=1$, IM

GO TO $(190,185,190)$, I GEP

$V O(I, J)=R 5(I) * Z 5(J) * V F$

GO TO 195

$190 \quad V O(I, J)=P I 2 * R 5(I) * 25(J) * R 4(I) * V F$

195 CONTINUE

200 CONTINUE

PRINT MIX INFORMATION AND RADII AND AXII INFORMATION

WRITE(NOUT, 204) (ID(I), I = 1,12$)$

204 FORMAT(1HI, $12 A 6 / / /)$

WRITE (NOUT, 248$)(\mathrm{J}, 10(J), I I(J), I 2(J), J=1, M 01)$

208 FORMAT 3 X, $16 \mathrm{H}$ MIXTURE NUMBER, $18 \mathrm{H}$ MIX COMMAND

$124 \mathrm{H}$ MATERIAL ATOMIC DENSITY $/(13,1 \mathrm{X}, \mathrm{I} 8,8 \mathrm{X}, \mathrm{I} 8,8 \mathrm{X}, \mathrm{E} 20.8)$ )

$Z Z=0.0$

$Z Z=0.0$
$I P=I M+I$

$J P=J M+1$

WRITE(NOUT, 65) (IUII), I $=1,12$

WRITEINOUT, 210

210 FORMAT $184 \mathrm{H}$

1 AXII

AVG AXII 1 )

AVG RADII

IF(IM - JM) $240,240,280$

240 WRITE(NOUT, 220$)(11, R I(I), R 4(I), 21(I), 24(I)), I=1$, IP

FORMAT $(I 4,4 \mathrm{~F} 20.4)$

IF (IM - JM) $241,500,241$

241 III $=I P+1$

WRITE(NOUT, 220$)(11, Z Z, Z Z, Z 1(1), Z 4(I)), I=I I I, J P$

GO TO 500

280 WRITE(NOUT, 220$)(11, R I(I), R 4(I), 21(I), 24(I)), I=1, J P)$ 



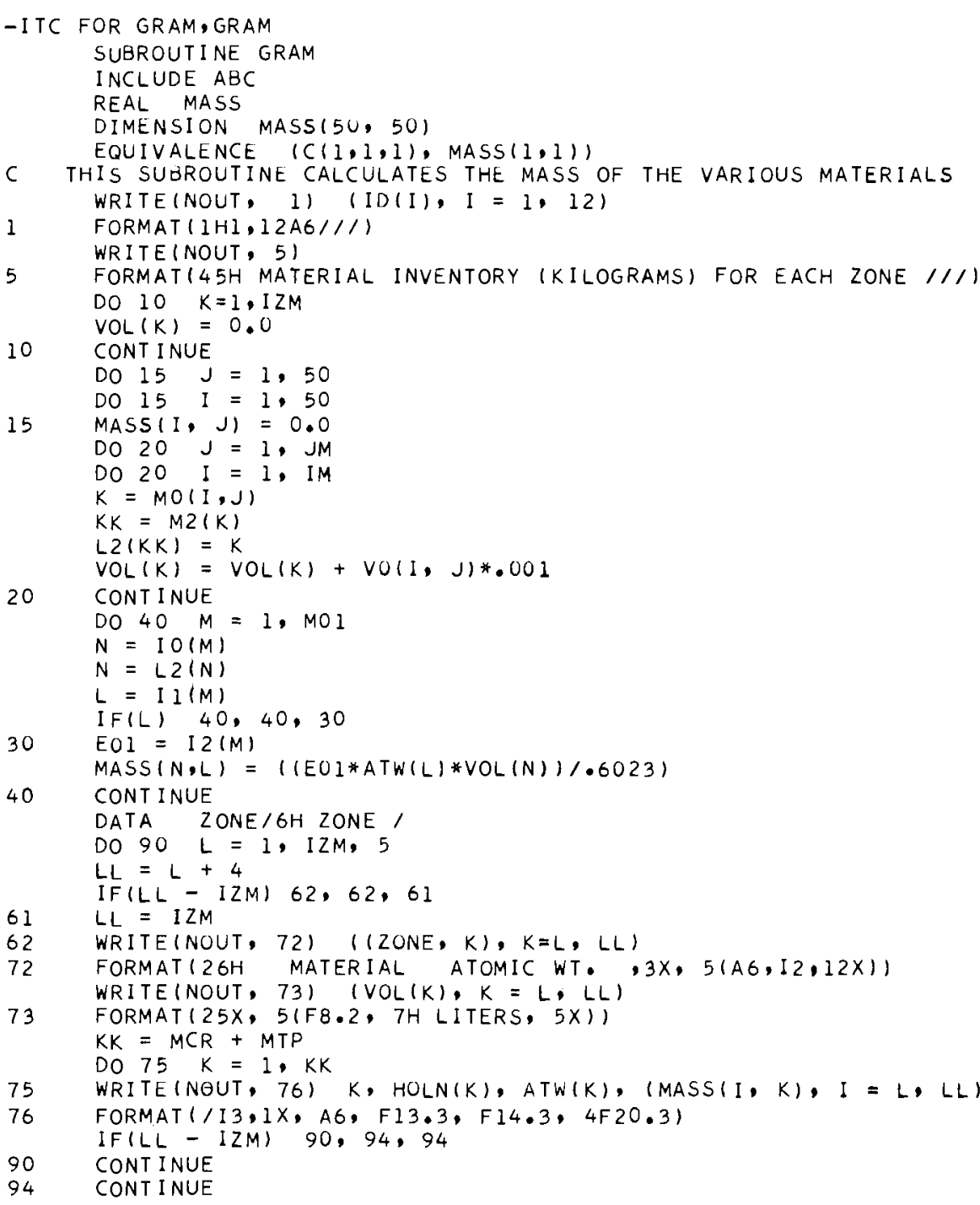




\section{-}

RETURN 
-ITC FOR NORM, NORM

SUBROUT I NE NORM

INCLUDE ABC

EQUIVALENCE (SORC $1(1,1)$, TPHI $(1,1))$, (SORC $2(1,1), C A D J(1,1))$,

C THIS SUBROUTINE NORMALIZES THE FLUXES IREGULAR AND ADJOINT)

$C F X=0.0$

$C P W=0.0$

POWER $=0.0$

DENOM $=0.0$

$R L=0.0$

$Z L=0.0$

RABS $=0.0$

RSORS $=0.0$

DO $10 J=1, J M$

DO $10 \mathrm{I}=1$, IM

$\operatorname{SORCI}(1, J)=0.0$

SORC $21, J)=0.0$

10 CONTINUE

REWIND NFLUX

REWIND NFLUXI

REWIND NSCRAT

REWIND NSCRAT

$\begin{array}{ll}\text { REWIND } & \text { NCRI } \\ \text { DO } 100 & K K=1, \text { IGM }\end{array}$

READINCR I $($ (COIII, J), I I $=1, I T L), J=1, M T)$

REAU (NFLUX) ( PHI I, J) I = , IM), J $=1, J M)$

READ (NFLUX2) (IADJ(I, J), I $=1, I M), J=1, J M)$

$C F X=C F X+P H I I I, 1$

$K=\operatorname{MO}(1,1)$

$K=M 2(K)$

$C P W=C P W+C O(1, K) * P H I(1,1)$

DO $20 \quad J=1, J M$

DO $20 \mathrm{I}=1$, IM

$K=$ MOII, Ji

$K=M 2(K)$

POWER = POWER + CU(I, K)*PHIII, J)*VO(I, J)

$\operatorname{SORCI}(I, J)=\operatorname{SORCI}(I, J)+\operatorname{CO}(3, K) * \operatorname{PHI}(I, J)$

$\operatorname{SORC2}(I, J)=\operatorname{SORC} 2(I, J)+C H I(K K) * A D J(I, J)$

RABS $=$ RABS + CO $(2, K) * P H I(I, J) * V O(I, J)$

RSORS $=$ RSORS + CO (3, K)*PHI (I, J)*VO(I, J)

20 CONTINUE

CONT I NUE
PI2 $=6.28318$

DO $50 \quad \mathrm{I}=12, \quad 28318$

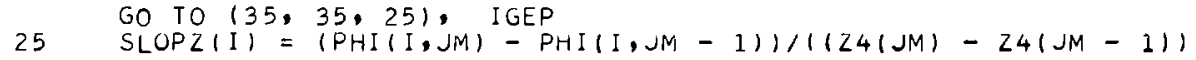

$1 * P I 2 * R 4$ (I) ) 
$S L O P Z(I)=(P H I(I, J M)-P H I(I, J M-1)) /(Z 4(J M)-Z 4(J M-1))$ CONT INUE

$K=M O(I, J M)$

$K=M 2(K)$

50

CONT I NUE

DO $60 \mathrm{I}=1$, IM

GO TO $(55,55,52)$, IGEP

$S L O P Z(I)=-(P H I(I, 2)-P H I(I, I))((24(2)-24(1)) * P I 2 * R 4(I))$

GO TO 56

SLOPZ (I)

CONT INUE

$K=M O(I, I)$
$K=M 2(K)$

$K=M 2(K)$

CONT INUE

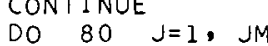

SLOPR (J) $=($ PHI (IM,J) - PHI (IM - I,J) ) (R4 IIM) - R4(IM - 1)

$K=\operatorname{MO}(I M, J)$

$K=M 2(K)$

$R L \quad=R L$

$+(S L O P R(J) * A 2(J)) /(3 . * \operatorname{CO}(4, K))$

100 CONTINUE

RLEAK $=-Z L-R L$
RSORS $=$ RSORS $/ V F$

RABS $=$ RABS/V

AK = (RSORSI)/RABS + RLEAK

$T S D=(M W-S E C) /(F I S S I O N S)$

$T S D=F E F * 1 \cdot 602 * 10 * *(-19)$

$C A D=\operatorname{SORC2}(1,1)$

$C P W=C P W * T S D * 1000$

POWER = POWER*TSD

REWIND NCRI

REWIND NFLUXI

REWIND NFLUX2

DO $200 \mathrm{~J}=1, J M$

DO $200 \quad J=1, J M$

DENOM $=$ DENOM + (SORC1 II, J) *SORC21 I, J)*VO(I, J) $) /(\operatorname{SORC2}(1,1) * A K)$

200 CONTINUE

GO TO $1240,260,280,3001$, NFP

240 CONST $=$ FLPO/CFX

GO TO 320

260 CONST $=\mathrm{FLPO} / \mathrm{CPW}$

GO TO 320

$\begin{array}{ll}\text { GO TO } & 320 \\ \text { CONST } & =\text { FLPO/POWER }\end{array}$

CONST = FLI

$\begin{array}{ll}\text { GO TO } & 320 \\ \text { CONST } & =\text { FLPO/DENOM }\end{array}$

300 CONST 
$C F X=C O N S T * C F X$

$C P W=C O N S T * C P W$

POWER = CONST*POWER

DENOM = CONST $*$ DENOM

WRITE (NOUT, 328) (ID(IK), IK = 1, 12)

328

RSORS = RSORS*VF*CONST

$R A B S=R A B S * V F * C O N S T$

RLEAK = RLEAK*VF*CONST

$R L=-R L * V F * C O N S T$

$Z L=-Z L * V F * C O N S T$

WRI TE (NOUT, 330$)$

330

, RL, ZL, AAK

RSORS, RABS, RLEAK

$144 \mathrm{H}$ CENTER POINT POWER (MWT/LITER)

$244 \mathrm{H}$ TOTAL POWER (MWT) - - -

$4 \quad 44 \mathrm{H}$ DENOMINATOR OF PERT. FORMULA

44H SOURCE RATE

44H ABSORPTION RATE - - - - - - - IPE12.5/1

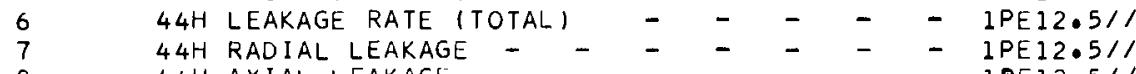

$844 \mathrm{H}$ AXIAL LEAKAGE - - - - - - - IPEI2.5/

DO 350 44 KEFF

DO $350 \quad J=I$, JM

DO $344 \mathrm{~N}=1$,

344 ACT II, J, N) $=0.0$

$T P H I(I, J)=0.0$

CADJII, J) $=0.0$

$\operatorname{POW}(I, J)=0.0$

350 CONTI'NUE

DO $400 \quad K=1, I G M$

READ (NFLUXI) ((PHI II, J), I = $1, I M), J=1, J M)$

READ (NFLUX2) ((ADJ $I, J), I=1, I M), J=1, J M)$

$\operatorname{READ}(N C R I)$ ( (CUIII, J), II $=1, I T L), J=1, M T)$

DO $360 \mathrm{~J}=1$, JM

$K K=M O(I, J)$

$K K=M 2(K K)$

$\operatorname{PHI}(I, J)=\operatorname{PHI}(I, J) * C O N S T$

$A D J(I, J)=A D J(I, J) / C A D$

$\operatorname{TPHI}(I, J)=\operatorname{TPHI}(I, J)+P H I(I, J$

CADJ(1, J) = CADJ(1, J) + CHI(K) *ADJ(I, J)

POW (I, J) = POW (1, J) + CO(1, KK)*PHI(I, J)*TSD*1000。

I F (NACT)

CONT INUE

DO $356 \mathrm{~N}=1$, NACT 
WRITE (NSCRAT) ((PHI(I, J), I $\pm I, I M), J=1, J M)$ WRITE(NSCRAT) ((ADJ II, J), I = I, IM), J $=1, J M)$ $M N=I F P+$

GO TO $(368,362,364), 1 M N$

PUNCH 363, ((PHI) I, J), I = I, IM), J = I, JM)

6ORMAT $(6 E 12 \cdot 6)$

GO TO 368

364 PUNCH 363, ((ADJ II, J), I = I, IM), J = I, JM)

368 IF (NPRT)

$376,376,370$

WRITE

WRITE (NOUT, 328)

WRITE (NOUT, 372) K

372 FORMATI/15H FLUX FOR GROUP, I3/)

CALL PRT(IM, JM, PHI)

WRI TE (NOUT, 328) (IDIIK), IK = 1, 12)

WRITE (NOUT, 374) K

374 FORMAT $/ / / 23$ H ADJOINT FLUX FOR GROUP, I3/

CALL PRT(IM, JM, ADJ)

CONTINUE

400 CONTINUE

WRITE(NOUT, 328) (ID (IK), IK $=1,12$ )

WRITE (NOUT, 478$)$

FORMATIIIH TOTAL FLUX/)

CALL PRT (IM, JM, TPHI)

WRI TE (NOUT, 328) (ID (IK), IK $=1,12)$

(

CALL PRT (IM, JM, CADJ)

WRITE(NOUT, 328) (ID(IK), IK $=1,12)$

WRI TE (NOUT, 482 I

482 FORMAT (26H POWER DENSITY (MWT/LITER) /

CALL PRTIIM, JM, POW

484 DO $488 \quad N=1$, NACT

DO $486 \mathrm{~J}=1, \mathrm{JM}$

DO $486 \mathrm{I}=1$, IM

486 SORC2 $(1, J)=A C T(I, J, N)$

WRITE(NOUT, 328) (ID(IK), IK = 1, 12)

$K K=M A(N)$

WRITE(NOUT, 487) N, KK, NN

487 FORMATI 9H ACTIVITY, I $3,13 \mathrm{H}$ ION POSITION,I3/) 


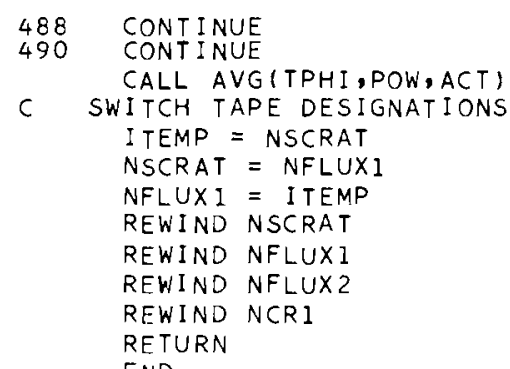

REWIND NFLUXI

REWIND NFLUX2

REWIND NCRI

RETURN 
-ITC FOR AVG, AVG

SUBROUTINE AVG(TPHI, POW, ACT)

INCLUDE $A B C$

C THIS SUBROUTINE CALCULATES zONE AVERAGED FLUXES, POWERS,

AND ACTIVITIES

DIMENSION ACT $(50,50,3)$, TPHI $(50,50)$, POW $(50,50)$

DATA CAT/6H ACT)

WRI TE (NOUT, 328) (ID (IK), IK $=1,12$ )

328 FORMAT $(1 \mathrm{H1}, 12 \mathrm{AG} / / / / / /)$

DO $340 \quad K K K=1, I Z M$

$Z P H I(K K K)=0.0$

$\begin{aligned} Z P O W(K K K) & =0.0\end{aligned}$

DO $340 \quad N=1,3$

$340 \quad Z A C T(K K K, N)=0.0$

DO $525 \quad J=1, J M$

DO $525 \quad I=1$, IM

$Z P H I(K K K)=Z P H I(K K K)+V O(I, J) * .001 * T P H I(I, J)$

ZPOW(KKK) = ZPOW

IF (NACT) $525,525,505$

505 DO $508 \quad N=1$, NACT

$508 Z A C T(K K K, N)=Z A C T(K K K, N)+V 0(I, J) * .001 * A C T(1, J, N)$

525 CONTINUE

DO $540 \quad K K K=1$, I ZM

$Z P H I(K K K)=Z P H I(K K K) / V O L(K K K)$

$Z P O W(K K K)=Z P O W(K K K) /$ VOL $(K K K)$

IF (NACT) $\quad 540,540,535$

535 DO $538 \quad N=1$. NACT

$538 Z A C T(K K K, N)=Z A C T(K K K, N) / V O L(K K K)$

540 CONTINUE

I F (NACT) $560,560,580$

WRI TE (NOUT 565 I
FORMAT $(48 \mathrm{H}$ ZONE
AV FLUX
AV POWER
$(1)$

DO 570 KKK $=1$, IZM KKK, ZPHI $(K K K), Z P O W(K K K)$

FORMAT $(1 / 16,5 E 20.7)$

GO TO 600

580 WRI TE (NOUT, 585) ((CAT,N), N=1, NACT)

585 FORMAT $48 \mathrm{H}$ ZONE $\quad$ AV FLUX

$13(A 6, I 1,13 X))$

DO 590 KKK $=1,12 M$

590 WRITE(NOUT, 575$)$ KKK, ZPHI $(K K K), Z P O W(K K K),(Z A C T(K K K, N), N=1, N A C T)$

CONT INUE

RETURN

END 
-ITC FOR ABETA, ABETA

SUBROUTINE ABETA

INCLUDE ABC

$B S I G F(50,50)$

EQUIVALENCE (PHR(1,1), BSIGF $(1,1)$

C THIS SUBROUTINE CALCULATES BETA EFFECTIVE AND NEUTRON LIFETIME WRITE(NOUT, 5$)$ (IDII), $1=1,12$

5 FORMAT $(1 \mathrm{H} 1,12 \mathrm{AG} / / / 1)$

DO $10 \mathrm{~J}=1,50$

DO $10 \quad I=1,50$

$\operatorname{SORCI}(1, J)=0.0$

$\operatorname{SORC} 2(I, J)=0.0$

PHZ(I,J) $=0.0$

$\operatorname{BSIGF}(I, J)=0.0$

10

CONT INUE

READ(NFLUXI) ( $P H I(I, J), I=I, I M), J=1, J M$

READ (NFLUXI) ( $(A D J(1, J), I=1,1 M), J=1, J M)$

$\operatorname{READ}(N C R 1) \quad((C \cup(I I, J), I I=1, I T L), J=1, M T)$

DO $500 J=1$, JM

DO $500 \quad=1, I M$

PHZ $(I, J)=(P H I(I, J) * A D J(I, J)) /(\operatorname{VEL}(K K))+P H Z(I, J)$

$K K K=M O(I, J)$

$K K K=M 2(K K K)$

DO $300 L=1$, NIBC

DEN $=0.0$

DO $120 \quad M=1$, MO1

IF (IO (M) $-K K K)^{M O 1} 120,110,120$

110 IF(II(M) - NBET(L)) 120, 115, 120

$115 D E N=D E N+12(M)$

120 CONTINUE

$N N=N B E T(L$

300 CONTINUE

C DELAYED NEUTRON SOURCE\#ADJOINT FLUX

CONTINUE

1000 CONTINUE

$B E=0.0$

ALIFE $=0.0$

DO $2000 \mathrm{~J}=1$, JM

DO 2000 I $=1$, IM

$B E=B E+5 O R C 2(1, J) *$

$\operatorname{BSIGF}(1, J) * V 0(1, J)$

2000 CONTINUE

ALIFE $=$ ALIFE/(DENOM*AK) 
WRFF $=$ BE/ $/$ DENOM*AK)
WRITE $(N O U T, 2050)$ BEFF, ALIFE

2050 FORMAT 143 H BETA EFFECTIVE

ALE - - - - - - 1PE11.5/1

RETURN 43 H NEUTRON GENERATION TIME - - - - - IPEII.51

END 
-ITC FOR CALC, CALC

SUBROUTINE CALC

INCLUDE ABC

INCLUDE ABC

EQUIVALENCE SLOAZ150

SLOAR $(50)$

1 (SLOAR(1), DUM(51)),

THIS SUBROUTINE USES PEI

TORTURBATION THEORY TO CALCULATE REACTIVITY THE

DO II I = 1, NDELK

REWIND NCR I

REWIND NFLUXI

REWIND NFLUX2

REW I ND NSCRAT
$N R=$ NROW(III)

$N R=N R O W($ I I I $)$

$N C=N C O L$ (I I I)

C CONVERSION FROM ATOMS TO KO

$C T=602.3 / A T W(M A T)$

$P I 2=6.28318$

DO $1 J=1,50$

DO $1 \quad I=1,50$

$\operatorname{SORC}(I, J)=0.0$

SORC $2(I, J)=0.0$

WRITE (NOUT, 2) (ID(I), I = 1,12 )

FORMAT $(1 \mathrm{HI}, 12 \mathrm{~A} 6 / / /)$

DO $1000 K=1, I G M \quad K=1, J), I=1, I M), J=1, J M)$

READ (NFLUXI) (CADJ(I,J), I = $1, I M), J=1, J M$

READ (NCRI) ((COIII, J), II = I, ITL), J = I, MT)

DO 3 II $=1$, ITL

3 CXIII, K) $=$ CO(II, MAT)

IF (NR) 400, 400, 10

DELTA K CALCULATION FOR ROW = NROW

CONT INUE

DO $60 \quad I=1$, IM

IF (NR - JM) $20,40,40$

$20 \quad N N=N R+1$

FLUX (1, K, I, 3) = PHI $(I, N N$

FLUX (2, K, I, 3) $=A D J(I, N N$

IF (NR - I) $50,50,40$

$40 \quad N N=N R-1$

FLUX $(1, K, I, 1)=\operatorname{PHI}(1, N N$

FLUX (2, K, I, I) $=A D J(I, N N$

$N N^{-}=N R$

FLUX $(1, K, I, 2)=P H I(I, N N)$

FLUX (2, K, I, 2$)=A D J(I, N N)$ 
SORC2 $(I, 1)=C H I(K) * F L U X(2, K, I, 2)+\operatorname{SORC2}(I, 1)$
SORCI I, 1$)=\operatorname{CO}(3, \operatorname{MAT}) * F L U X(1, K, I, 2)+\operatorname{SORC} 1(1,1)$

SORCI $(1,2)=C O(2, M A T) * F L U X(1, K, 1,2) * F L U X(2, K, 1)$

SORC1

601 CONTINUE

CONTINUE

DO $150 \quad I=1, I M$

PSRI $=($ FLUX $(1, K, I, 2)-F L U X(1, K, I-1,2)) /(R 4(I)-R 4(I-1))$

$P S R 1=(F L U X(1, K, I, 2)-F L U X(1, K, I-1,2)) /(R 4(I)-R 4(I-1))$
$A S R I=(F L U X(2, K, I, 2)-F L U X(2, K, I-1,2)) /(R 4(I)-R 4(I-1))$

$\operatorname{IF}(1-I M) 78,86,86, I+1,2)-F \operatorname{PSR}(1, K, I, 2)) /(R 4(I+1)-R 4(I))$

$A S R 2=(F L U X(2, K, I+1,2)-F L U X(2, K, I, 2)) /(R 4(I+1)-R 4(I))$

GO TO 90

SLOPR (I) $=0.0$

$\operatorname{SLOAR}(I)=0.0$

GO TO 92

SLOPR (I) $=$ PSR 1

SLOAR(I) = ASRI

GO TO 92

$S L O P R(I)=(P S R 1+P S R 2) / 2 \cdot 0$

$S L O A R(I)=(A S R 1+A S R 2) / 2.0$

CONT INUE

IF (NR - 1) $100,100,93$

$93 \quad$ GO TO $(96,96,94)$, IGEP

(

$1 * P I 2 * R 4$ (I) )

$A S Z 1=(F L U X(2, K, I, 2)-F L U X(2, K, I, 1)) /((Z 4(N R)$

$1 * P I 2 * R 4$ (I))

GO TO 97

PSZ $1=(F L U X(1, K, I, 2)-F L U X(1, K, I, 1)) /(24(N R)$

$A S Z)=(F L U X(2, K, I, 2)-F L U X(2, K, I, I)) /(24(N R)$

97 IF(NR -IM) $100,115,115$

102 PSZ2 $=(F L U X(1, K, 1,3)-F L U X(1, K, I, 2)) /((24(N R+1)-24(N R))$

1 *PI 2 *R 4 (I))

$A S Z 2=(F L U X(2, K, I, 3)-F L U X(2, K, I, 2)) /((Z 4(N R+1)-Z 4(N R)$

$1 * P$ I $2 * R 4$ ( I) )

GO TO 106

105 PSZ2 $=(F L U X(1, K, I, 3)-F L U X(1, K, I, 2)) /(Z 4(N R+1)-24(N R))$

$A S Z 2=(F L U X(2, K, I, 3)-F L U X(2, K, I, 2)) /(Z 4(N R+1)-24(N R))$

I F (NR-1) $108,108,12$

$1 C 8$ SLOPZ(1) $=$ PSZ2

$S L O A Z(I)=A S Z 2$

GO TO 130

$-24(N R-1))$

$-24(N R-1))$

$-24(N R-1))$

$-24(N R-1))$

CONT INUE

IF (NR - IM) $120,115,115$

115 SLOPZ(I) = PSZ1 


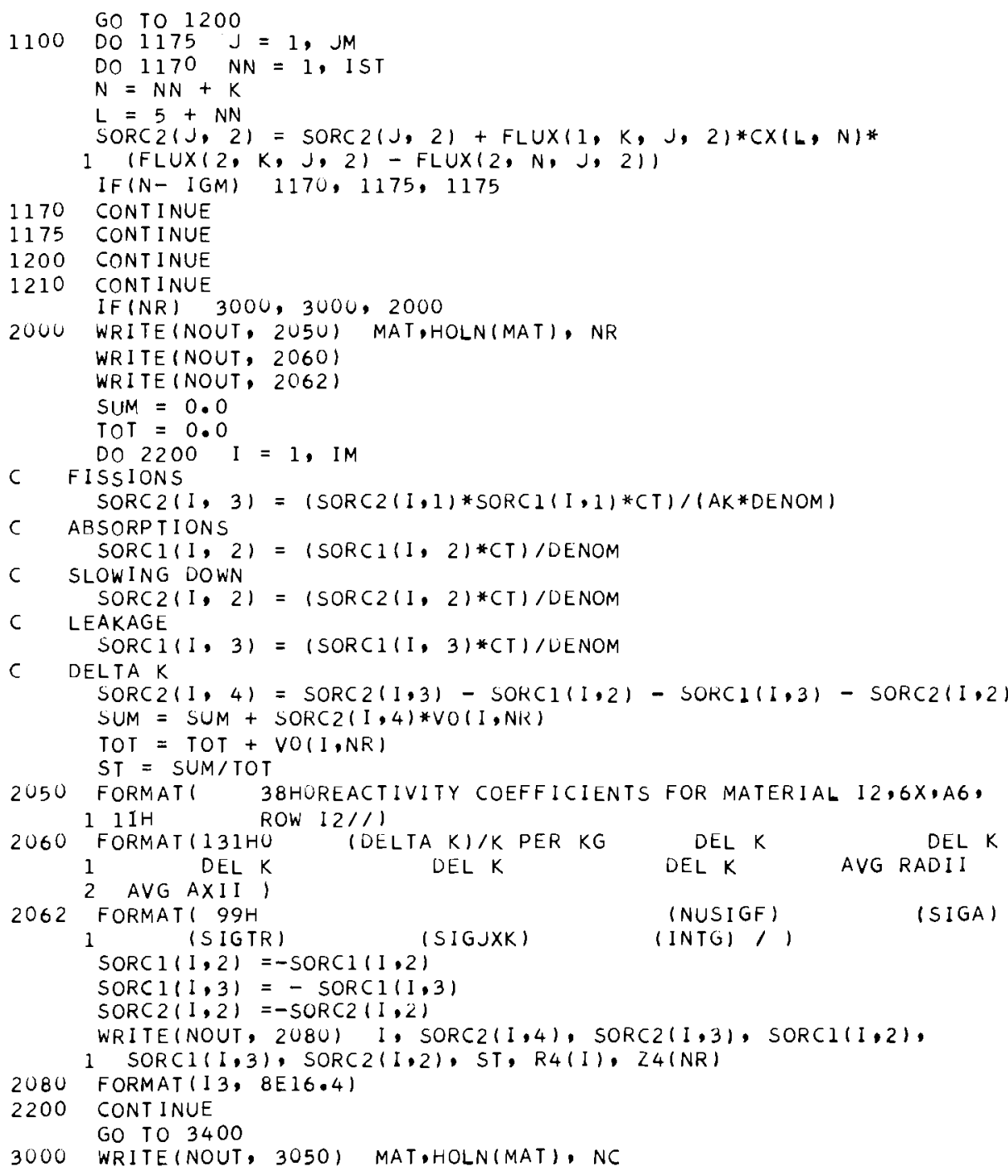


WRI IE (NOUT, 2060 )

WRITE (NOUT, 2062

SUM $=0.0$

DO $3200 \mathrm{I}=1$, JM

c

ISSIONS

SORC $2(1,3)=(\operatorname{SORC} 2(1,1) * \operatorname{SORC} 1(1,1) * C T) /($ AK*DENOM)

C ABSORPTIONS

SORC $1(1,2)=(\operatorname{SORC} 1(1,2) * C T) / D E N O M$

C SLOWING DOWN

C LORC2(I, 2) $=($ SORC2 $(1,2) * C T)$ DDENOM

SORC $1(1,3)=($ SORCIII, 3$) * C T) / D E N O M$

C DELTAK

$\operatorname{SORC} 2(1,4)=\operatorname{SORC}(I, 3)-\operatorname{SORCI}(I, 2)-\operatorname{SORC}(1,3)-\operatorname{SORC}(I, 2)$

SUM = SUM + SORC2 $(I, 4) * V O(N C, I)$

TOT $=$ TOT + VO $(N C, I)$

$S T=S U M / T O T$

3050 FORMATI 38HOREACTIVITY COEFFICIENTS FOR MATERIAL I2, 6X,A6,

$114 \mathrm{H}$

COLUMN I 211 (1)

SORC $1(9,2)=-$ SORCI $(1,2)$

SORC $1(1,3)=-\operatorname{SORC} 1(1,3)$

SORC $2(1,2)=-\operatorname{SORC} 2(1,2)$

WRITE(NOUT, 2080) I, SORC2(I,4), SORC2(I,3), SORCI (I, 2)

3200 CONTINUE

3400 CONTINUE
9999 CONTINUE

REWIND NCR

REWIND NFLUXI

REWIND NFLUX2

REWIND NSCRAT

RETURN

END 
BNWL- 409

\section{DISTRIBUTION}

Number of Copies
Argonne National Laboratory

R. A. Jaross

LMFBR Program Office

Senior RDT Site Rep.

Atomic Energy Commission, Richland, Washington

P. G. Holsted, Senior RDT Site Rep.

Atomic Energy Commission, Washington

Division of Reactor Development \& Technology

M. Shaw, Director, RDT

Asst. Dir. for Nuclear Safety

Analysis \& Evaluation Br, RDT:NS

Environmental \& Sanitary Engineering Branch, RDT:NS

Research and Development Branch, RDT:NS

Asst. Dir. for Plant Engineering Branch, RDT

Applications \& Facilities Branch, RDT:PE

Components Branch, RDT:PE

Instrumentation $\xi$ Control Branch, RDT:PE

Systems Engineering Branch, RDT:PE

Asst. Dir. for Program Analysis, RDT

Asst. Dir. for Project Management, RDT

Liquid Metals Projects Branch, RDT:PM

FFTF Project Manager, RDT:PM (3)

Asst. Dir. for Reactor Engineering

Contro1 Mechanisms Branch, RDT:RE

Core Design Branch, RDT:RE

Fuel Fabrication Branch, RDT:RE

Fuel Handling Branch, RDT:RE

Reactor Vessels Branch, RDT:RE

Asst. Dir. for Reactor Technology

Chemistry \& CHemical Separations Branch, RDT:RT (2)

Fuels \& Materials Branch; RDT:RT

Reactor Physics Branch, RDT:RT

Special Technology Branch, RDT:RT

Atomics International

L. E. Glasgow

R. W. Dickinson

Senior RDT Site Rep. 
$\checkmark$

Number of Copies

2

1

1

5

1

1

5

1
Atomic Power Development Associates

B. V. D. Farris, Contract Administrator

Senior RDT Site Rep.

Babcock and Wilcox Company

S. H. Esleeck

Babcock and Wilcox Company

Sterling Avenue

Barberton, Ohio 44203

T. P. Farre11

Division of Technical Information Extension

General Atomic Division

D. Coburn

General Electric Company, San Jose

Senior RDT Site Rep.

General Electric Company, Sunnyvale

Dr. K Cohen, General Manager (3)

C. P. Schively, c/o Bertram Wolfe

Bertram Wolfe

Idaho Nuclear Corporation

D. R. de Boisblanc

Idaho Operations Office

C. W. Bi11s, Director

Rich 1 and Operations Office

J. H. Krema

C. L. Robinson

R. K. Sharp

Technical Information Library

San Francisco Operations Office

Director, Reactor Division 
Stanford University

$$
\text { Dr. R. Sher }
$$

Westinghouse Electric Corporation

Dr. J. C. R. Kelly, General Manager

Battelle Northwest

E. R. Astley

W. J. Bailey

J. M. Batch

R. E. Bardsley

A. L. Bement

R. A. Bennett

J. R. Boldt

C. L. Boyd

D. C. Boyd

C. L. Brown

W. L. Bunch

C. A. Burgess

M. P. Burns

C. P. Cabe 11

J. L. Carter

W. L. Chase

T. 1). Chikalla

T. 'l'. člaudison

P. :. Conn

R. R. Cone

D. L. Condotta

T. J. Crocker

R. E. Dah 1

J. M. Davidson

D. R. Doman

G. E. Driver

R. V. Dulin

S. L. Engstrom

P. L. Farnsworth

L. M. Finch

G. L. Fox

C. D. Flowers/M. T. Jakub

R. L. Gibby

S. M. Gill

J. E. Hanson

R. W. Hardie (30)

R. A. Harris

H. Harty

R. A. Harvey 
Battelle Northwest, (Contd)

B. R. Hayward

J. W. He lm

L. G. Henke

R. J. Hennig

J. Herb

C. W. Higby

P. L. Hofmann

P. G. Holsted

R. L. Knecht

D. J. LaGrou

D. D. Lanning

H. D. Lenkersdorfer

W. R. Lewis

C. W. Lindenmeier

H. E. Little

W. W. Little

R. J. Lobsinger

L. L. Maas

M. K. Mahaffey

W. B. McDonald

M. H. Meuser

M. K. Millhollen

D. M. Nero

R. E. Nightingale

W. M. Noble

M. G. Patrick

A. Padilla

L. A. Pember

R. E. Peterson

F. G. Richards

W. E. Roake

R. E. Schenter

F. H. Shadel

D. W. Shannon

R. E. Sharp

B. O. Shaver

D. E. Simpson

R. J. Squires

G. H. Strong

C. D. Swanson

G. Thieme

J. P. Thomas

G. L. Tingey

W. L. Townsend

K. G. Toyoda

D. M. Walley

A. E. Waltar

J. H. Westsik 
Batte11e Northwest, (Contd)

L. A. Whinery

R. D. Widrig

M. F. Wiitala

M. R. Wood

F. W. Woodfie1d

J. M. Yatabe

H. H. Yoshikawa

Technical Information Files

Technical Publications (2) 